\title{
DEVELOPMENT OF AN INFRARED ABSORPTION SPECTROSCOPE BASED ON LINEAR VARIABLE FILTERS
}

\author{
A Thesis \\ by \\ FELIPE GUIMARÃES NOGUEIRA
}

\author{
Submitted to the Office of Graduate Studies of \\ Texas A\&M University \\ in partial fulfillment of the requirements for the degree of \\ MASTER OF SCIENCE
}

December 2006

Major Subject: Computer Science 
DEVELOPMENT OF AN INFRARED ABSORPTION SPECTROSCOPE BASED ON LINEAR VARIABLE FILTERS

\author{
A Thesis \\ by \\ FELIPE GUIMARÃES NOGUEIRA
}

\begin{abstract}
Submitted to the Office of Graduate Studies of Texas A\&M University

in partial fulfillment of the requirements for the degree of

MASTER OF SCIENCE
\end{abstract}

Approved by:

Chair of Committee,

Committee Members,

Ricardo Gutiérrez-Osuna

Gerard L. Coté

Dezhen Song

Head of Department,

Valerie E. Taylor

December 2006

Major Subject: Computer Science 


\begin{abstract}
Development of an Infrared Absorption Spectroscope Based on Linear Variable Filters.

(December 2006)

Felipe Guimarães Nogueira, B.S., Texas A\&M University

Chair of Advisory Committee: Dr. Ricardo Gutiérrez-Osuna
\end{abstract}

The objective of this thesis is to develop a low-cost infrared absorption spectroscope based on linear variable filter (LVF) technology for the automated detection of gases and vapors, and the semi-automated detection of liquids. This instrument represents an alternative to electronic-nose instruments based on cross-selective gas sensor arrays. Instead, the proposed instrument uses the idea of computational "pseudo-sensors", whereby spectral lines in an analytical instrument are clustered into groups and used as independent variables. We characterize the system on a number of performance metrics, uncovering its detection limits and resolving power. We present calibration methods to estimate the concentration of analytes in a matrix of absorbing species, as well as signal processing techniques for spectral classification. Specifically, we validate the instrument on a mixture calibration problem with simple and complex chemicals, and compare the efficiency of different calibration methods to estimate the concentration of one analyte in the matrix. Moreover, we demonstrate the use of the instrument on two real-world applications in the foodstuffs domain: oil adulteration and trans fatty acid (TFA) detection. The instrument, combined with signal processing techniques, is able to fully 
discriminate oils, as well as classify margarine and spreads onto high-TFA and low-TFA groups. Despite operating at a low spectral resolution, our results show that the LVF based spectroscope is a promising alternative to traditional analytical techniques for selected niche applications. 


\section{DEDICATION}

To my wife, Martha. 


\section{ACKNOWLEDGMENTS}

First and foremost, I would like to thank Dr. Ricardo Gutiérrez-Osuna for introducing me to this exciting field of research, and for his superb guidance and generosity.

I cannot fully express my gratitude to the exceptional team at the Pattern Recognition and Intelligent Sensor Machine Lab. They are Daniel Felps, Jobany Rodríguez, Agustin Gutiérrez-Gálvez, Barani Raman, Jongyoon Choi and Takao Yamanaka. Special thanks to Daniel Felps for his generous assistance and for his immeasurable contribution to the initial phase of this project. My thanks also to Jobany Rodríguez for all the insights gained as a result of our numerous and ephemeral brainstorming sessions. For their generous assistance to this research, I would like to acknowledge Dr. Gerard Coté and Dr. Dezhen Song.

My gratitude also to my parents, Roberto and Solange Nogueira, and to my other parents, Gwen and Gary Williams, for being role models, for always believing, and for unselfishly and lovingly helping fulfill my dreams. Furthermore, I praise Nina, for many cherished moments of distraction. Finally, I would like to thank the most extraordinary woman in my life, my precious wife, Martha, for her enduring patience and support. 


\section{TABLE OF CONTENTS}

Page

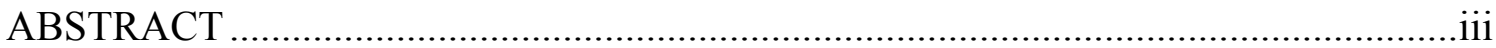

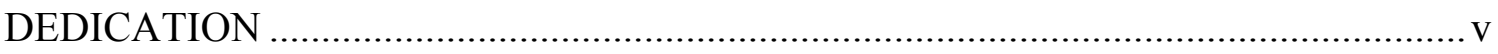

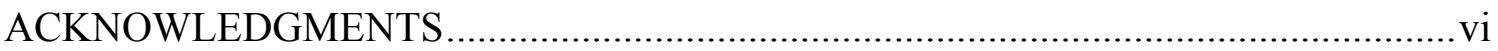

TABLE OF CONTENTS .................................................................................... vii

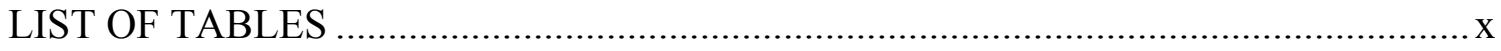

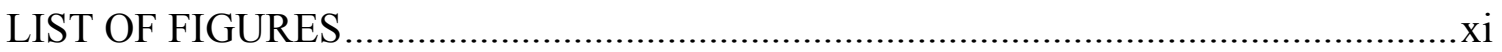

\section{CHAPTER}

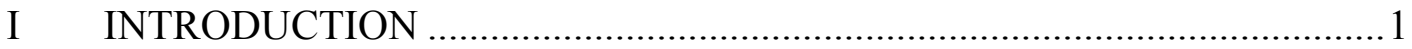

I.1. Organization.......................................................................

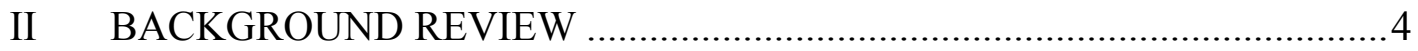

II.1. Fundamental principles of infrared absorption spectroscopy ............4

II.1.1. Electromagnetic radiation...........................................4

II.1.2. Infrared absorptions and normal modes of vibration...........6

II.2. Infrared system components ................................................... 10

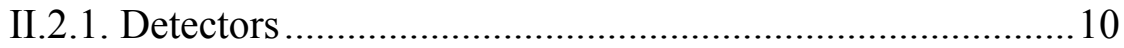

II.2.2. IR sources ............................................................. 18

II.2.3. Gas cell ................................................................ 19

II.3. Infrared system configurations ............................................20

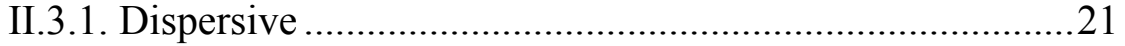

II.3.2. Interferometric ................................................... 22

II.4. Infrared spectroscopy methods ................................................23

II.4.1. Transmission........................................................ 23

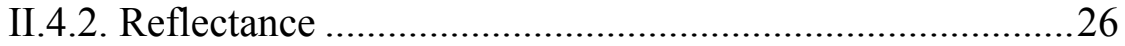

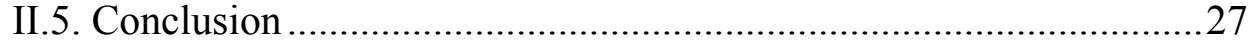

III INFRARED INTEGRATED SYSTEM ...............................................29

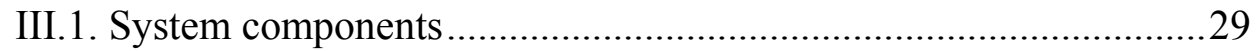

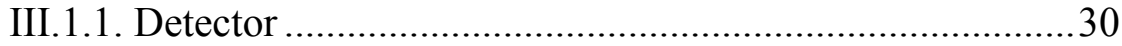




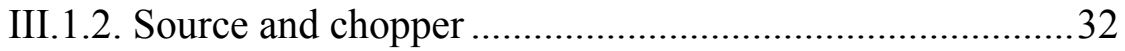

III.1.3. Chamber and windows ........................................................

III.1.4. Pump, valves, samples and other components ..................34

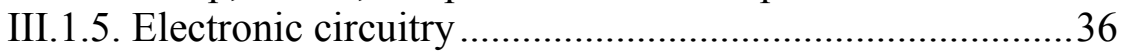

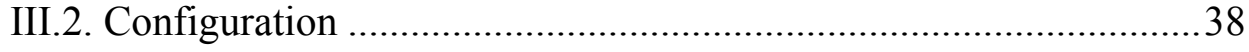

III.3. Characterization ...................................................................... 41

III.3.1. Performance metrics and analysis ....................................42

III.3.2. Detection limits and sensitivity ..........................................

III.3.3. Resolving power............................................................ 51

III.3.4. Resolution and intensity losses .....................................52

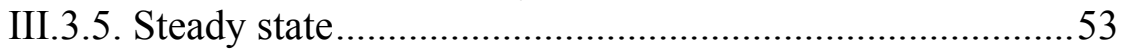

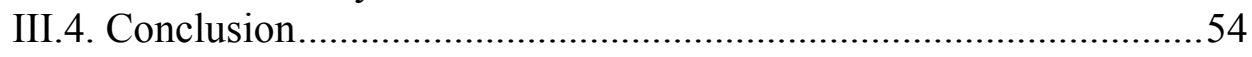

IV SPECTRAL SIGNAL PROCESSING......................................................55

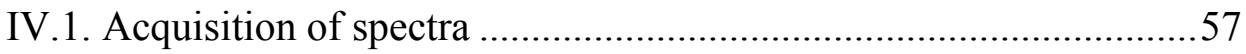

IV.1.1. Chemical modulation with lock-in amplification .............57

IV.1.2. Signal integration ..........................................................58

IV.1.3. CM/LA vs. integration time …………..........................5

IV.1.4. Difference spectra ........................................................59

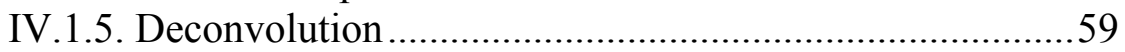

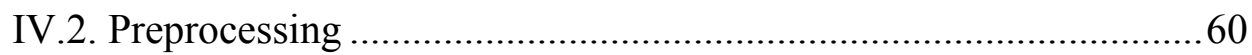

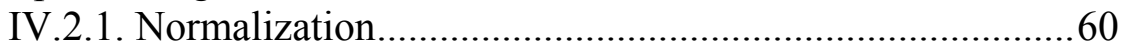

IV.3. Feature selection and extraction.................................................62

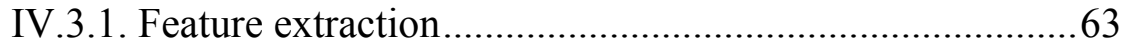

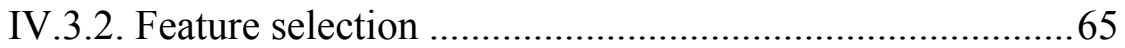

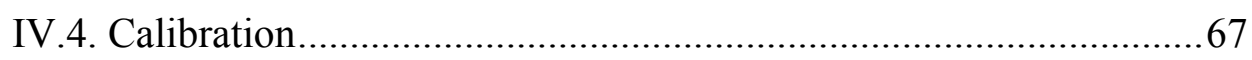

IV.4.1. Classical least squares (CLS) ..........................................69

IV.4.2. Inverse least squares (ILS) …………............................ 70

IV.4.3. Principal component regression (PCR) ...........................71

IV.4.4. Partial least squares regression (PLSR) .............................73

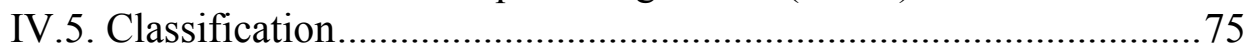

IV.5.1. Quadratic classifiers.......................................................76

IV.5.2. K-nearest neighbors $(\mathrm{KNN})$........................................... 77

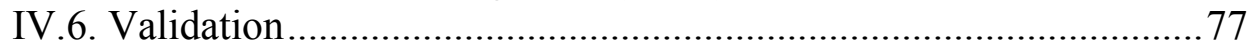

IV.6.1. Leave-one-out cross-validation (LOO) ...........................78

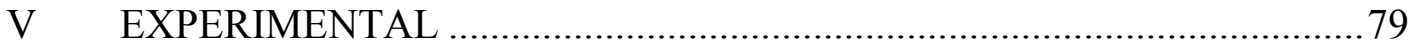

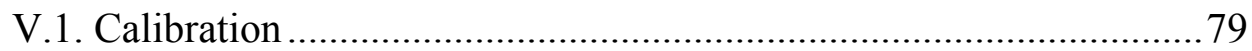

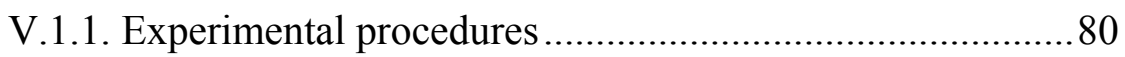

V.1.2. Spectra of the mixture components ..................................... 80 
CHAPTER Page

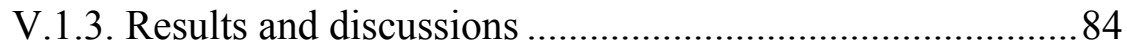

V.2. Edible oils and fats ............................................................... 95

V.2.1. The mid infrared spectra of oils and fats ........................98

V.2.2. Oils ..................................................................... 100

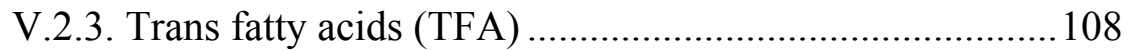

V.3. Conclusion........................................................................ 115

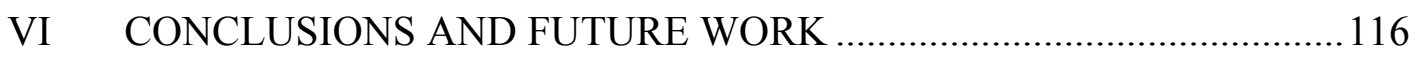

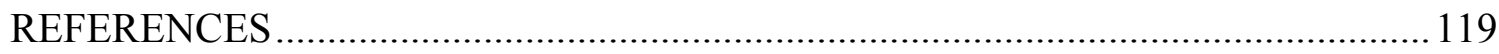

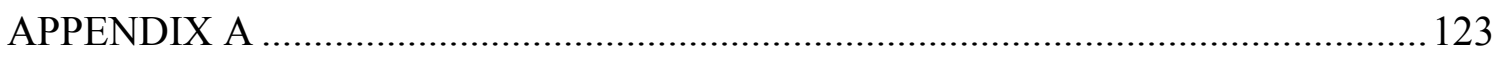

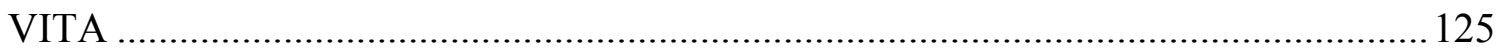




\section{LIST OF TABLES}

TABLE

1 Common IR transmitting window materials. Adapted from Workman Jr., 1998

2 Mixture composition. The values in each column below the analytes indicate the percentage by weight of each component in the corresponding mixture.

3 Twenty five important frequency bands and shoulders of edible oils in the mid infrared region, along with the assigned functional group, mode of vibration and the intensity. Adapted from Guillen \& Cabo, 1997

4 Composition of the samples as provided by the producers

$5 \quad$ Samples' producers

$6 \quad$ Selected features and their corresponding 1NN/LOO classification rates obtained from PLMR feature selection for the chosen preprocessing techniques. The PLMR parameters are $\mathrm{L}=4$ and $\mathrm{R}=3$.

$7 \quad$ Samples' producers, type and class assignment. 110

8 Composition of the samples as provided by the manufacturers 


\section{LIST OF FIGURES}

FIGURE $\quad$ Page

1 The electromagnetic spectrum in different units (Schmidt, 2005) .................6

2 Conceptual division of the mid IR spectra of Hexanol into functional

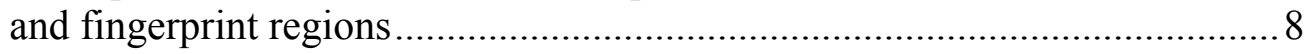

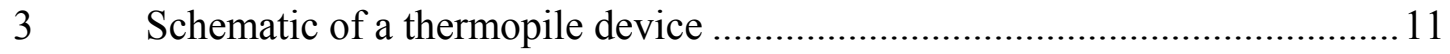

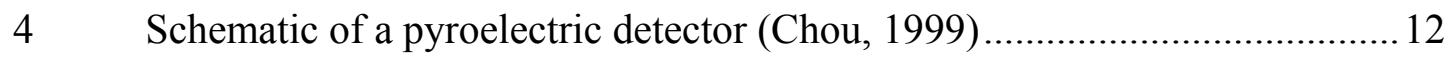

$5 \quad$ Schematic of a Luft detector (Chou, 1999) ................................................. 14

6 Schematic of a photoacoustic detector (Chou, 1999) ................................. 15

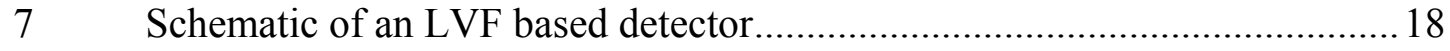

8 Diagram of the infrared radiation path in a dispersive infrared spectrometer. Adapted from ("FT-IR vs. Dispersive Infrared " 2002) .......... 21

9 Path of the infrared beam in a Fourier Transform Infrared (FTIR) spectrometer. Adapted from ("FT-IR vs. Dispersive Infrared " 2002) .......... 23

10 Simple schematic of the absorption by transmission method (Workman Jr., 1998)

11 Simple schematic of the spectral reflection method for infrared absorption spectroscopy (Workman Jr., 1998) ..........................................22

12 High-level schematic diagram; P: pump, R: reference gas, S: samples, PS: pressure sensor, G: gas cell, C: mechanical chopper, and D: detector.

13 Top-left: Photograph of an LVF. Top-right: a diagram of the infrared radiation path as it passes through the LVF and reaches a detector array. Bottom-left: a display of the linear wavelength output characteristic of the LVF. Bottom-right: rendering of the LVF's physical aspects (JDSU, 2006) 
14 Schematic of the infrared source (model IR-12K from Boston

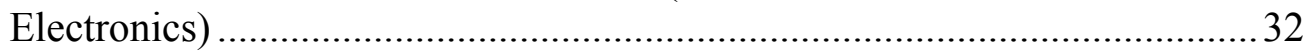

15 Top: photograph of two gas cells of different lengths. Bottom: a gas cell's component arrangement (model Beta Gas Cell from Buck

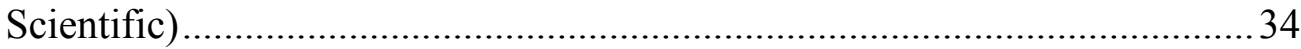

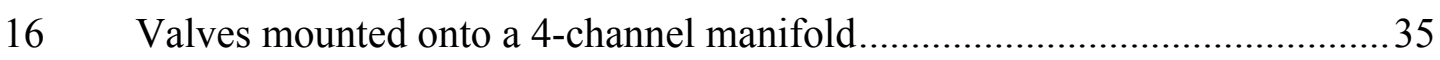

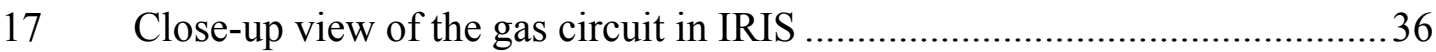

18 Pressure sensor signal amplification circuit. PS 01 and PS 02 are the differential pressure sensor output. The values of the resistors R1 and $\mathrm{R} 2$ are $1 \mathrm{~K} \Omega$ and $100 \mathrm{~K} \Omega$, respectively. DAQ I2 and DAQ I3 are the amplified differential input to the DAQ. The operation amplifier is a standard 741

19 Valve driver circuit. DO is the DAQ output control for the valves. V1 and V2 are the output of the circuit. Note that V1 and V2 are operated simultaneously. The operation amplifier used is a 741; the MOSFET is an ECG261.

20 Synoptic of IRIS. The user controls IRIS via a graphical user interface installed on personal computer. The personal computer sends control signals to the instrumentation electronics, which allows for seamless manipulation of the infrared system and gas circuit components. The gas circuit begins at the air inlet of a diaphragm pump, and continues through a 4 valve manifold. The valve system selectively chooses the flow path, allowing only a particular or a combination of samples to be in the gas circuit. The headspace of the sample container(s) is pushed to a gas cell and pressurized. A pressure sensor enables precise pressurization by providing real-time acquisition of the pressure level inside the gas cell. The radiation emitted by the infrared source is transmitted and absorbed, at certain wavelengths, by the gas sample, modulated by the chopper, selectively filtered by the LVF and measured at the detector array 
21 IRIS software interface. On the left, the device status panel displays the state of the pump and valves, and allows their manual control. The pressure indicator displays real-time information about the pressure level inside the gas chamber. On the top-center, the virtual spectroscope displays the intensity of the signal captured at each wavelength in the detector array. On the bottom-center, the tabbed panel allows for rapid access to the three execution modes available (i.e. scripted execution, manual execution, automated execution) and to the configuration settings

22 Raw signal measurements over an approximately 15-hour period. Random levels of noise, represented as sudden changes in detected intensity, can be observed

23 Relative uncertainty by pixel, calculated by dividing the expanded uncertainty by the mean of the measurements.

24 IRIS absorption detection limits.

25 Average of 47 measurements of the infrared absorption spectra of VOC components for 1:100 dilution of acetone in water at 12 psi. Notice that, in order to obtain just the peaks that are due to acetone, we have taken water vapor as a reference spectrum

26 Signal intensity as a factor of source-detector distance. Adapted from (Passerini et al., 2003)...........................................................................52

27 False color image of raw signal response after initial power-up....................53

28 Stages of a pattern recognition problem (Gutierrez-Osuna, 1998) ................55

29 Infrared absorption spectrum of acetone in gas phase. i) Highresolution spectrum obtained via FTIR. Adapted from NIST (Linstrom \& Mallard, 2003). ii) Infrared absorption spectrum obtained by IRIS

30 Infrared absorption spectrum of isopropyl alcohol in gas phase. i) Highresolution spectrum obtained via FTIR. Adapted from NIST (Linstrom \& Mallard, 2003). ii) Infrared absorption spectrum obtained by IRIS

31 Infrared absorption spectrum of water in gas phase. i) High-resolution spectrum obtained via FTIR. Adapted from NIST (Linstrom \& Mallard, 2003). ii) Infrared absorption spectrum obtained by IRIS 
32 Infrared absorption spectrum of witch hazel in gas phase obtained by IRIS. FTIR spectra was not available at the NIST site

33 Infrared absorption spectra of thirteen mixtures with dissimilar proportions of acetone, alcohol, witch hazel and water obtained by IRIS

34 Estimated concentration of acetone from the mixtures described in Table 2 using classical least squares calibration and leave-one-out cross-validation.

35 Estimated concentration of acetone from the mixtures described in Table 2 using inverse least squares calibration and leave-one-out crossvalidation

36 Average behavior of mean square error (MSE) estimate as a function of the number of principal components obtained for PCR via double loop LOO cross validation

37 First four principal component loadings

38 Correlation between principal component score vectors and true concentration of acetone

39 Estimated concentration of acetone from the mixtures described in Table 2 using principal component regression calibration and leaveone-out cross-validation.

40 Average behavior of the mean square error (MSE) estimate as a function of the number of latent vectors obtained for PLSR via double loop LOO cross validation

$41 \quad$ First four latent vectors .93

42 Correlation coefficient between PLSR score vectors and true concentration of acetone

43 Estimated concentration of acetone from the mixtures described in Table 2 using partial least squares regression calibration and leave-oneout cross-validation 
44 i) Saturated fatty acid. ii) Unsaturated fatty acid in cis configuration. iii) Unsaturated fatty acid in trans configuration.

45 Infrared absorption spectrum of olive oil in the mid infrared region. i) Spectrum obtained by a high resolution FTIR spectroscope (Guillen \& Cabo, 1997). ii) Spectrum obtained by IRIS. The numbers atop each major peak indicates the frequency band assignment in 0 .

46 Infrared absorption spectra of five edible oils obtained in IRIS 103

47 Spectra of edible oils normalized to unit vector.

48 Spectra of edible oils after subtracting the infrared spectrum of peanut oil

49 Second derivative spectra of edible oils 105

50 Second derivative at 968 versus $1110 \mathrm{~cm}^{-1}$

51 Ratio of absorption $A_{20} / A_{21}$ (See Table 3), which is indicative of the percentage by weight of polyunsaturated acyl groups in edible oils 108

52 Two types of spread packaging. i) Tub ii) Stick

53 Infrared absorption spectra of margarine and spread samples. Dashed and solid lines represent samples in stick and tub packages, respectively. Three replicates per sample are shown

54 Infrared absorption spectra of margarine and spread samples normalized to a unit vector

55 TFA infrared absorption band of margarine and spreads after the subtraction of the infrared spectrum of soybean oil 


\section{CHAPTER I}

\section{INTRODUCTION}

Arrays of cross-selective chemical sensors, commonly referred to as electronic noses, have been developed during the past two decades as a low-cost alternative to the analysis of volatile compounds (Persaud \& Dodd, 1982). The e-nose approach is very effective when one wishes to obtain a holistic response to the sample. However, since the detection principle in these instruments is combinatorial (i.e., many volatiles are detected by a single sensor, and vice-versa), it becomes challenging to determine which component in a mixture is responsible for the response of the array. Unless a proxy can be found for the measurand of interest (i.e., the target analyte is correlated with an easily measurable variable), the results of e-nose instruments can be misleading. For instance, in a now classical study (Pinheiro et al., 2001) it was shown that the response of an electronic nose to the headspace of wine during the fermentation process is due to the alcohol content of the wine rather than its aroma. Further, electronic noses are limited to the analysis of volatile species, which may have a different composition than the liquid (or solid) sample that one is interested in analyzing.

In analogy with the electronic-nose paradigm, an array of "pseudo-sensors" may be obtained by selectively clustering the response of an analytical instrument according to its independent variable (i.e., retention time in a gas chromatograph, absorption wavelengths in a Fourier Transform Infrared Spectrometer (FTIR), or mass/charge ratios

This thesis follows the style of Biological Cybernetics. 
in a mass spectrometer) (Nagle et al., 1998). To date, the use of this approach has been limited to laboratory settings due to the cost, and size of the instruments. Advances in micro- and nano- fabrication technology are, however, likely to overcome these issues (Bacon et al., 2004; Badman \& Cooks, 2000; Taylor et al., 2003). Along these lines, (Rubio et al., 2004) have shown that an IR detector can be coupled with a Fabry-Perot tunable filter to produce a miniaturized IR spectrometer. In a related article, the authors propose to use an optical filter array atop a thermopile array (Rubio et al., 2005). Among the advantages of this approach over traditional electronic-nose instruments are interpretability (i.e. the ability to correlate spectral features with molecular structure) and contact-free sensing, which helps prevent sensor drift. In this thesis, we present a lowcost instrument based on infrared spectrometry.

In contrast with these previous efforts, the objective of this thesis is to develop a system based on a linear variable filter (LVF), which acts as a bank of optical filters, and a pyroelectric detector array, for the automated detection and characterization of chemical gas and liquid chemicals. The advantage of this approach is that it can be easily interpreted as a low-resolution IR spectrum. On the down side, the sensitivity of this device can be significantly lower than that of e-nose sensors. Among the many challenges of LVF based spectroscopy are the relatively low signal-to-noise ratio and the reduced resolving power of the instrument, which make the characterization and classification of similar compounds difficult. In order to overcome these challenges, we provide several signal processing techniques, particularly for data acquisition and the associated data preprocessing. We complement the discussion of the signal processing 
methods by providing techniques for calibration, which consists of building predictive mathematical models for concentration of an analyte in a matrix of absorbing species, and classification of spectral patterns via statistical machine learning techniques. Finally, we show a comparison between popular calibration methods for the data acquired by the instrument, and demonstrate the use of the instrument on two real-world foodstuff applications.

\section{I.1. Organization}

The remaining sections of the thesis are organized as follows. Chapter II provides a review on the fundamentals of infrared absorption spectroscopy, as well as an overview of traditional and innovative infrared system components, configurations and methods. A description of the proposed system, as well as the system characterization is covered in Chapter III. Chapter IV discusses processing techniques for calibration and spectral classification. Chapter V provides a calibration example and an evaluation of the system for two real-world applications in the foodstuffs domain. Finally, we conclude the thesis by pointing out future directions for research in Chapter VI. 


\section{CHAPTER II}

\section{BACKGROUND REVIEW}

In this chapter, we briefly discuss the fundamental principles of infrared absorption spectroscopy (Schilz, 2000; Schmidt, 2005; Stuart, 2004), and give an overview of the current (Chou, 1999; Passerini et al., 2003) and future technology (Andresen \& Fulop, 2005) that have motivated this research.

\section{II.1. Fundamental principles of infrared absorption spectroscopy}

\section{II.1.1. Electromagnetic radiation}

Since the introduction of Maxwell's classical theory of electro- and magneto-dynamics in 1864 , we have been able to interpret electromagnetic radiation beyond the visible spectrum. According to Maxwell's equations, radiation is considered as two mutually perpendicular electric $\left(E^{\prime}\right)$ and magnetic $(H)$ fields, oscillating in single planes to each other (Stuart, 2004). The vector product $S=E^{\prime} \times H$ is called Poynting vector, and follows the direction of energy flow. A complete sinusoidal cycle along $S$ is referred to as the wavelength (i.e. energy) of an electromagnetic traversal wave (i.e. light).

Plank explained that energy $E$ relates proportionally to frequency $v$, with a proportionality constant $\left(h=6.626 \times 10^{-34} J s\right)$ as follows

$$
E=h \times v
$$


Light propagates in a vacuum at a constant speed $c=2.997925 \times 10^{8} \pm 3 \mathrm{~m} \mathrm{~s}^{-1}$, and relates wavelength $\lambda$ and frequency $v$ such that $c=v \times \lambda$ (Schmidt, 2005). Thus, energy can be expressed as

$$
E=h \times \frac{c}{\lambda}
$$

or, in terms of its reciprocal length, i.e. wave number $k=\frac{1}{\lambda}$, as

$$
E=h \times c \times k
$$

Vibration and rotation of atoms and molecules can be represented by discrete energy levels. A stream of particles, i.e. quanta, of energy interacts with matter by either absorption or emission. However, in order for interaction to occur, a quantum of energy must exactly fit between neighboring energy levels (Stuart, 2004). For instance, given two neighboring atomic energy levels $E_{1}$ and $E_{2}$, absorption occurs when frequency is given by

$$
v=\frac{\left(E_{2}-E_{1}\right)}{h}
$$

The electromagnetic radiation spectrum ranges, in wavelength, from millions of kilometers to fractions of femtometers, and is conventionally divided in many regions, as illustrated in Fig 1. In terms of wavelength, the infrared region spans from $1 \mathrm{~mm}$ to 750nm, and is divided into three sub-regions: near (i.e. 0.75-5 $\mu \mathrm{m}$ ), mid (i.e. 5-30 $\mu \mathrm{m}$ ) and far (i.e. 30-1000 $\mu \mathrm{m}$ ) infrared. The near infrared region is closest to the visible spectrum, whereas the far infrared, as the name implies, is farthest. Most of the discussion from here on will be concerned with the mid infrared region. Care must be 
taken when using these infrared sub-region definitions, since there is no international standard for these specifications, and authors often disagree on the subject.

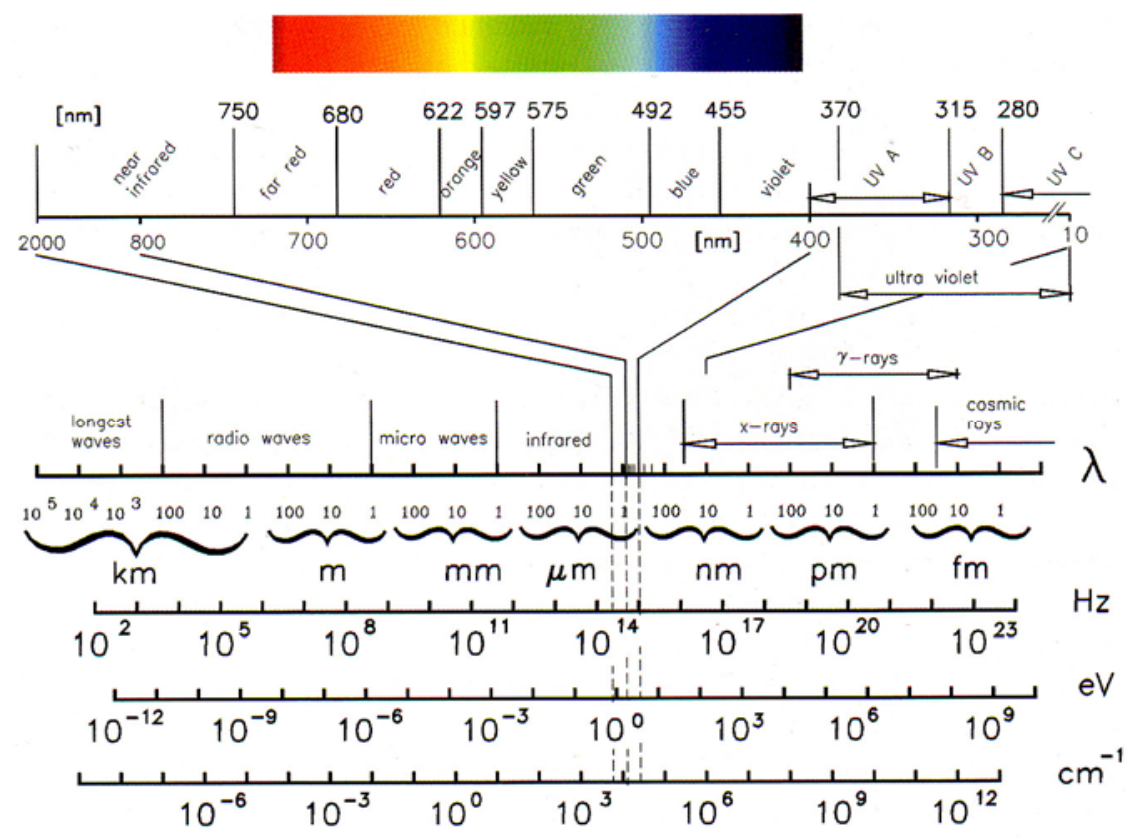

Fig 1. The electromagnetic spectrum in different units (Schmidt, 2005).

\section{II.1.2. Infrared absorptions and normal modes of vibration}

Fundamentally, the field of infrared absorption spectroscopy strides to determine the differences in energy caused by the absorption of infrared radiation when reflected or transmitted through a medium. In order for infrared absorption to occur, energy must cause a change in a molecule's electric dipole moment. This assertion is referred to as the selection rule. The selection rule implies that only heteronuclear diatomic or polyatomic molecules may show absorption in the infrared region.

Changes in the dipole moments of a polyatomic molecule can be considered in terms of molecular vibrations and rotations. These movements occur according to the 
molecule's degrees of freedom. There are basically three types of degrees of freedom: translational, rotational and vibrational. The total number of a molecule's degrees of freedom is the sum of translational, rotational and vibrational degrees of freedom. For any molecule with $\mathrm{N}$ atoms, it follows that the total number of degrees of freedom it possesses is $3 \mathrm{~N}$. A general rule of thumb is that, since larger molecules have more degrees of vibration, they generally have many more peaks of absorption then smaller molecules. In addition, bonds of atoms that are farther away in the periodic table shows stronger bands than the contrary because they have stronger dipole momentum (Stuart, 2004).

Each molecule produces a unique infrared absorption spectrum because of its particular bonds and structures. A mid infrared absorption spectrum of a molecule may be conceptually divided into two regions, namely the functional-group region and the fingerprint region. To illustrate this idea, Fig 2 shows the plot of the mid infrared spectrum of Hexanol, with the marked division between functional and fingerprint regions. As with the determination of near, mid and far infrared regions, there is currently no international standard or governing body to establish a set of specification to define these regions. The functional region is considered to have absorption bands due to molecular functional group bonds, whereas the fingerprint contains the response to the intrinsic skeletal vibrations, mostly unique to each molecule. 


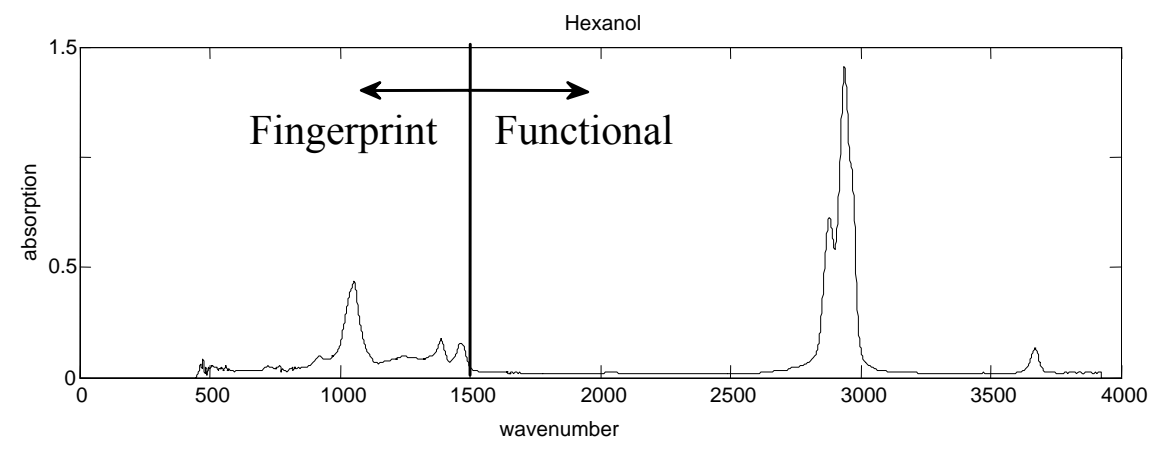

Fig 2. Conceptual division of the mid IR spectra of Hexanol into functional and fingerprint regions.

The division of the infrared spectrum into functional and fingerprint regions is important to analytical spectroscopy. The functional region typically offers more general information about a certain compound's chemical structure than the fingerprint region. By performing spectral analysis of the principal bands found in the functional region, we are generally able to correlate the sample to functional groups, which is particularly useful in the preliminary steps of molecular identification. On the other hand, infrared absorption in the fingerprint region of molecules that belong to the same functional group can be quite different. Peaks in the fingerprint region are very useful for classification, or in the last steps of molecular identification.

There are many factors that can make experimental infrared spectroscopy less than straightforward, and which can complicate a successful interpretation of the spectrum. When first observing infrared absorption profiles, one may easily realize that absorption bands aren't infinitely narrow. Broadening of bands can be attributed to many 
factors such as collision between molecules, finite lifetime of state transitions, time varying energy states or the Doppler effect (Stuart, 2004).

Experimentally, spectral absorption lines of molecules may vary tremendously because of solvents, temperature and other environmental factors in place. Different temperatures may cause fluctuation in the number of vibrational and rotational degrees of freedom. For instance, at low temperatures one can selectively freeze degrees of rotational freedom (Schmidt, 2005). Solvents may interact with the molecule, also causing a change in a molecules' absorption lines. Nonetheless, in most cases, the major absorption lines of a given molecule are still apparent regardless of the solvent used or temperature changes.

In addition, there are other complicating factors such as overtone, combination bands, Fermi resonance, coupling and vibration-rotation bands. Overtone bands are integer multiples of the fundamental frequency, which is proportional to wavenumber. A spectral measurement may show energy absorbed at a fundamental frequency of a band as well as at its overtone. Combination bands are additive in the sense that if a molecule possesses bands at two different fundamental frequencies, it may show absorption of energy at a frequency equivalent to the sum of these two fundamental frequencies. On the other hand, a Fermi resonance may split an absorption band in two when an overtone frequency exists in the same or similar region as a fundamental frequency. Coupling occurs when adjacent atoms in a molecule have similar frequencies, which lead to a change in overall frequency of the bands. Vibration-rotation bands occur when rotational motion is induced by a vibrational transition (Stuart, 2004). 
These complicating factors must be taken in account when selecting or designing an infrared absorption spectroscope. Fortunately, there is a great variety of components that allows enormous flexibility in the design and configuration of infrared systems. In the next section, we discuss the current and future infrared system component technologies.

\section{II.2. Infrared system components}

Most infrared absorption spectroscopes share a common set of components: an infrared source, a sample chamber and a detector. In this section we provide an overview of the most common components, and discuss their functionality. This discussion is not intended as a complete reference; further information about this and other components can be found at (Andresen \& Fulop, 2005; Rogalski, 2002; Schilz, 2000; Schmidt, 2005; Workman Jr., 1998).

\section{II.2.1. Detectors}

Although infrared detector materials and technologies have evolved tremendously in the past few decades, their basic principles of operation remain very similar. Fundamentally, infrared light is sensed by using either a photon or a thermal detector. Examples of photon-based sensing are photovoltaic, photoconductive, photodiode, photoemissive and photoelectromagnetic detectors. Thermal-based sensing of infrared light is obtained, for instance, by employing thermoelectric, thermoresistive or thermoconductive detectors. Furthermore, although the denomination of certain infrared detectors (e.g. photoacoustic or optopneumatic) may suggest the use of disparate techniques, their 
fundamental detection principles still lies or is complemented by thermal or photon interaction with sensing materials. In this section we briefly discuss the fundamental principles of most popular photon and thermal infrared detector technologies on the market today.

\section{II.2.1.1. Thermoelectric detector}

The building blocks of thermoelectric detectors (i.e. thermopiles) are thermocouples: devices that convert temperature into electrical signals. A thermocouple is made up of junctions of different metals, which generate a voltage potential directly proportional to temperature. As illustrated in Fig 3, there are two junctions in a thermocouple. One junction is made sensitive to radiant flux, while another is coupled with a heat sink material to provide a reference. Inside a thermopile is a series of miniaturized thermocouples arranged to obtain an improved output signal. In terms of infrared detectors, the thermopile is the simplest technology. It offers a slow response, DC stability, no need for a bias and sensibility to all wavelengths (Chou, 1999).

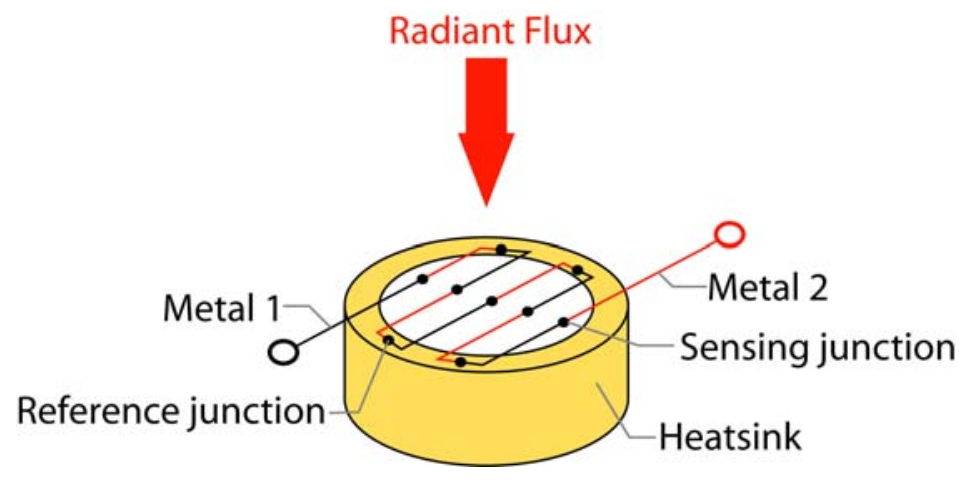

Fig 3. Schematic of a thermopile device. 


\section{II.2.1.2. Thermistor bolometer detector}

Thermistor bolometers are semiconductor devices that change resistance when exposed to infrared radiation. Infrared radiation interacts with the sensor by causing the fluctuation of surface temperature, which is detected by a sintered metal oxide material with high temperature coefficient of resistance.

\section{II.2.1.3. Pyroelectric detector}

Pyroelectric detectors are made of crystals, such as lithium tantalate, which exhibit spontaneous polarization or a concentrated electric charge that is temperature dependent (Chou, 1999). Changes in temperature generated by incident infrared radiation causes a flow of current that is proportional to the intensity of radiation. Pyroelectric detectors are more sensitive than thermoelectric or thermistor bolometer detectors. In addition, they show much larger current output and temperature stability, which allows for easier interaction with electronic circuits. The basic inner workings of a pyroelectric sensor are illustrated in Fig 4 below.

\section{PYROELECTRIC}

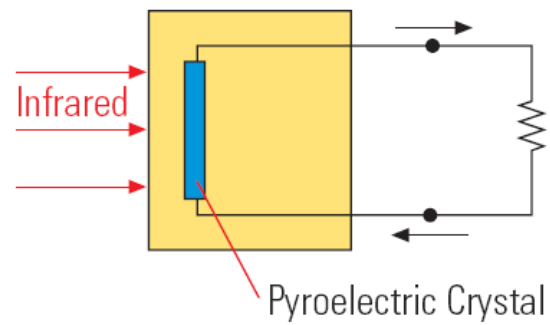

Fig 4. Schematic of a pyroelectric detector (Chou, 1999). 


\section{II.2.1.4. Photoconductive detector}

This type of device detects the effects of a quantum of energy (i.e. photon) incident on a semiconductor material. As seen previously, the energy of a photon is based upon its wavelength; photons with shorter wavelengths possess more energy than those with longer wavelengths. A photon with sufficient energy can excite an electron from a nonconducting to a conducting state. By examining the difference in conductivity, a photon detector is able to determine the number of photons in a pulse of monochromatic light. This type of sensor typically targets specific frequencies and requires cooling in order to function properly. In general, photon detectors are employed when high performance is required, such as medical bio-imaging or certain military infrared target detection applications.

\section{II.2.1.5. Luft detector}

Named after its inventor Karl Luft, the Luft detector is an optopneumatic detector. It examines the difference in pressure between a zero gas cell - which is inert to infrared radiation - and a sample gas cell when exposed to infrared radiation at wavelengths that cause absorption. By analyzing the pressure difference, a Luft detector is able to determine the concentration of the analyte in the sample cell. This pressure-based device typically employs either a diaphragm or a small passage between the chambers to measure pressure difference. In the case of a diaphragm, pressure is measured as changes in capacitance caused by the movement of the diaphragm due to unequal pressure between the chambers. In the second approach, a micro-flow sensor is placed at a small passage to measure detectable flows when pressures between chambers are distinct. This 
detector finds many applications as an analyzer, but may suffer from external factors such as small vibrations and temperature changes in the environment. A micro-flow based Luft detector is illustrated in Fig 5.

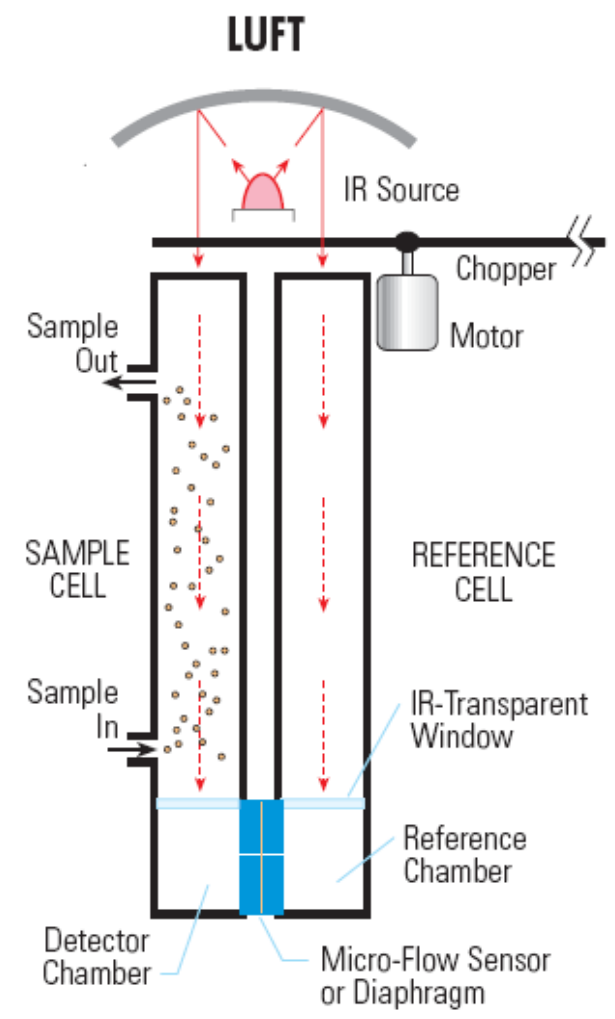

Fig 5. Schematic of a Luft detector (Chou, 1999).

\section{II.2.1.6. Photoacoustic detector}

A photoacoustic detector detects intermittent infrared radiation incident in a sample. Temperature changes caused by absorption produce a transient pressure response that is captured by a microphone or piezoelectric device. The system injects the sample gas inside a sealed compartment with a window capable of infrared transmission. An external infrared source emits pulses that penetrate the window, are absorbed by the 
sample, and are measured as oscillating pressure changes by the photoacoustic detector. The transduction principles used in photoacoustic detector is illustrated in Fig 6.

\section{PHOTOACOUSTIC}

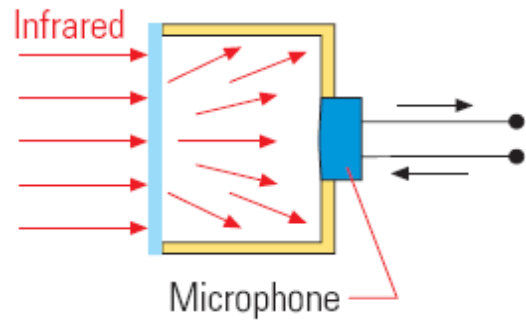

Fig 6. Schematic of a photoacoustic detector (Chou, 1999).

\section{II.2.1.7. New trends in infrared detector technology}

In the past decade, there have been world-wide efforts to develop new and improved infrared detection technologies for a number of commercial and military applications. Two-dimensional sensing of multi-color (i.e. multi-spectral) thermal radiation has been the focus of much of this research. In addition, commercial applications such as analytical spectroscopy have motivated the development of simpler and cost-effective technologies for room-temperature thermal sensing, which has inspired this research. In this section we discuss two important infrared detection technologies, focal plane arrays and linear variable filter based pyroelectric detector array, which have emerged, as a result of high performance and low-cost application demands, respectively. 


\section{II.2.1.7.1. Focal plane array (FPA)}

Applications in security, defense and biotechnology have been major targets of recent research in infrared detection technology. In the field of defense and security, applications have stressed a need for multi-band operation for simultaneous long-range detection and identification of threats, detection of low-contrast targets, improved warning against missile threats, defeating counter measures and the detection of recently buried mines (Andresen \& Fulop, 2005). Needs such as these have led to the development of what is known as the $3^{\text {rd }}$ generation in infrared detectors: focal plane arrays (FPA) with high pixel count and simultaneous dual band operation.

Most FPAs are arrays of photon detectors based on variable gap semiconductor technologies, which are manufactured using band gap engineering techniques. Fundamentally, these techniques consist of carefully blending semiconductor and semimetal materials, such that the band gap between valence and conducting state of the resulting alloy can be precisely specified to suit a specific range of wavelengths of the infrared electromagnetic spectrum. The main materials used in this technology today are HdCdTe and GaAIAs/GaAs QWIP (quantum well infrared photoconductor), but the adoption of Type-II InAs/GaInSb superlattice (T2SL) in future technology is expected (Andresen \& Fulop, 2005).

Thermal detector technologies are also present in some FPA devices (e.g. microbolometers), and but they have been, until recently, less common. This detector technology was popularly believed to be much slower and less sensitive in comparison with photon detectors, and therefore it was much less exploited for commercial or 
military purposes. As a result, efforts in the widespread development of thermal detectors for FPAs were exceptionally small (Rogalski, 2002). Today, however, this technology has been shown to provide fast imagery at room-temperature at rates of 30 frames per second. Nonetheless, for high-performance applications, thermal detection is still inferior to photon detection based FPAs.

FPA detectors are often categorized by whether or not they require cooling. Typically, cooled FPAs are based on photon detection technologies, whereas uncooled FPAs are based on thermal detectors. FPA detectors that require coolers for proper operation typically offer superior sensitivity and higher frame rates. On the other hand, uncooled FPA devices are generally lighter, smaller, more shock-resistant, powerefficient and have lower costs. However, despite the advantages of uncooled FPAs, their cost remains too high for widespread proliferation (Andresen \& Fulop, 2005).

\section{II.2.1.7.2. LVF and $\mu$ Array}

The need for simpler solutions for analytical measurements has prompted the development of uncooled infrared detectors, an example of which is the LVF spectrometer used in this thesis (Passerini et al., 2003). The device consists of a linear variable filter (LVF) placed in front of an array of 64 pyroelectric sensors ( $\mu$ Array; IR Microsystems, Lausanne, Switzerland), as illustrated in Fig 7. The LVF serves as a bank of optical band-pass filters, screening the spectra such that each sensor in the 64-pixel detector receives different wavelengths. This detector offers affordable, reliable, rugged, simple to use, room temperature spectrometry in the mid infrared region at the cost of lower resolution and lower sensitivity. The resolution power of the detector is limited by 
the number of pixels in the array and the optical properties of the LVF. In addition, sensitivity is lower because the LVF diffuses a considerable amount of radiation before it reaches the detector. In this work, we characterize a system based on this technology, and present signal-processing techniques that address issues of low resolution, low sensitivity spectrometry.

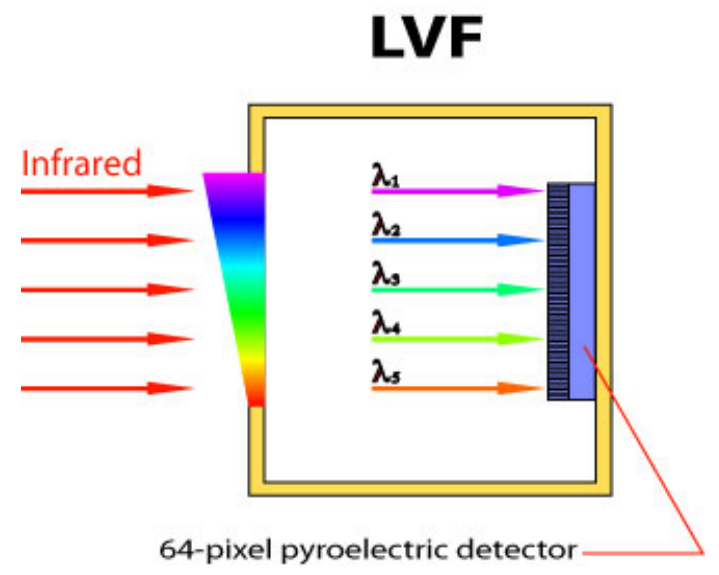

Fig 7. Schematic of an LVF based detector.

\section{II.2.2. IR sources}

Light sources can be categorized as continuum or line radiators (Chou, 1999). A continuum radiator, as the name suggests, provides radiation distributed across a wide spectral range, whereas a line radiator provides radiation at specific set of distinct spectral lines. Examples of continuum and line radiators are incandescent lights and lasers, respectively. The determination of which type of source, and what characteristics it should possess, depends invariably on the target application. The most important characteristic of a type of radiator is intensity. Light may be measured as energy in units of Watt seconds. The intensity of irradiation, known as radiant flux $\phi_{e}$, is defined by the 
quotient of energy over time - and can be measured simply as Watts. Any regular incandescent light source emits infrared radiation, and a source as small as a pen flashlight provides enough radiation for the detection of most hydrocarbons, $\mathrm{CO}_{2}$ and CO (Chou, 1999). However, most system configurations for analytical measurements require potent, i.e. high intensity, sources to avoid saturation of the signal acquisition equipment. The intensity distribution generally shows a maximum with specific halfwidth, and the shape of the energy distribution depends on what type of emitter is used (Schmidt, 2005).

\section{II.2.2.1. Modulation}

Modulation of the infrared radiation emission is often desired or required. For instance, pyroelectric sensors require modulation in order to define a basis for change in current flow. Intensity modulation is typically achieved through voltage modulation at the emitter or through a mechanical chopper positioned in the light path.

\section{II.2.3. Gas cell}

Gas cells can be found in various shapes and formats, and are designed for use with specific sampling methods. In transmission sampling techniques, the length of the cell determines the path length in which the sample is subject to absorption. The amount of radiation absorbed is proportional to the path length. Typically, small cells (e.g. $10 \mathrm{~cm}$ in length) are intended for use with concentrated gases, gas mixtures and vapors $(>1 \%$ levels) at ambient temperatures and pressures. Infrared radiation enters or leaves the gas cell through windows, which are made of a special material intended to have to the least 
absorption possible at the wavelengths under observation. A list of these materials is provided in Table 1. In addition, the selection of the window depends on the type of solvent and sample to be used in the experiments, as well as the operating conditions (e.g. shock or temperature).

The detection of trace gases (e.g. 1-1000 ppm) in diluted mixtures typically requires much longer gas cells, with path length in the range of 1-20 meters. For environmental applications, such as the detection of toxic gases in a chemical plant, gas cells are replaced by optical equipment with telescopic properties, and path lengths of several hundreds of meters are used (Workman Jr., 1998).

Table 1 Common IR transmitting window materials. Adapted from Workman Jr., 1998.

\begin{tabular}{lccc}
\hline \multicolumn{1}{c}{ Material } & $\begin{array}{c}\text { Useful range } \\
\left(\mathrm{cm}^{-1} ; \text { transmission }\right)\end{array}$ & $\begin{array}{c}\text { Refractive Index at } \\
1000 \mathrm{~cm}^{-1}\end{array}$ & $\begin{array}{c}\text { Water Solubility } \\
\left(\mathrm{g} / 100 \mathrm{ml}, \mathrm{H}_{2} \mathrm{O}\right)\end{array}$ \\
\hline Sodium chloride $(\mathrm{NaCl})$ & $40,000-590$ & 1.49 & 35.7 \\
Potassium bromide $(\mathrm{KBr})$ & $40,000-340$ & 1.52 & 65.2 \\
Cesium iodide $(\mathrm{CsI})$ & $40,000-200$ & 1.74 & 88.4 \\
Calcium fluoride $\left(\mathrm{CaF}_{2}\right)$ & $50,000-1,140$ & 1.39 & Insoluble \\
Barium fluoride $\left(\mathrm{BaF}_{2}\right)$ & $50,000-840$ & 1.42 & Insoluble \\
Silver bromide $(\mathrm{AgBr})$ & $20,000-300$ & 2.2 & Insoluble \\
Zinc sulfide $(\mathrm{ZnS})$ & $17,000-833$ & 2.2 & Insoluble \\
Zinc selenide $(\mathrm{ZnSe})$ & $20,000-460$ & 2.4 & Insoluble
\end{tabular}

\section{II.3. Infrared system configurations}

The infrared system components mentioned in section II.2 can be complemented with other optical or mechanical devices, and arranged to form complete spectroscopy systems. The most typical infrared system configurations for spectroscopy are dispersive and interferometric. In this section we discuss the fundamental principles of such systems. 


\section{II.3.1. Dispersive}

Dispersive infrared instruments, also referred to as grating or scanning spectrometers, measure dispersed infrared radiation obtained via dispersive gratings. The instrument typically utilize a grating monochromator, which allows scanning of the spectrum by mechanically rotating an element, which blocks all wavelengths except for the ones of interest. Illustrated in Fig 8, a dispersive infrared spectrometer consists of a source, reference chamber, chopper, grating monochromator, slit, detector and a set of mirrors. The source emits infrared radiation simultaneously to a reference and a sample cell, where absorption occurs. Both infrared beams go to a chopper that lets through only one beam at a time. After passing the chopper, an individual beam reaches a grating monochromator, which allows passage of certain wavelengths selectively to the detector.

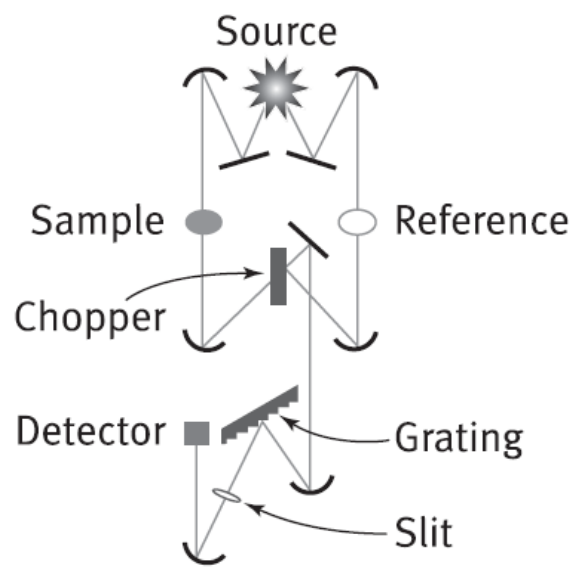

Fig 8. Diagram of the infrared radiation path in a dispersive infrared spectrometer. Adapted from ("FT-IR vs. dispersive infrared " 2002). 


\section{II.3.2. Interferometric}

In contrast, an interferometric device measures the temporal coherence of an incident infrared radiation beam. As illustrated in Fig 9, an interferometric system consists of a source, a beamsplitter, two mirrors, a laser and a detector. Light travels from the source to the beamsplitter where it is split into two parts. The beamsplitter causes one part to be transmitted to a stationary mirror, and another to be reflected from a moving mirror that oscillates at a constant velocity according to a very precise laser. The moving mirror makes the two beams travel different distances, causing an interference pattern to be created when they recombine at the beamsplitter. After leaving the beamsplitter, the pattern is sent through the sample, where absorption occurs, to be detected at the sensor all at once. After the signal is acquired, a computer is used to perform a Fourier transform on the interference pattern to convert it to a single beam spectrum. In addition, a reference signal must be collected for the calculation of absorption using BeerLambert's Law (See Section II.4.1.1). 


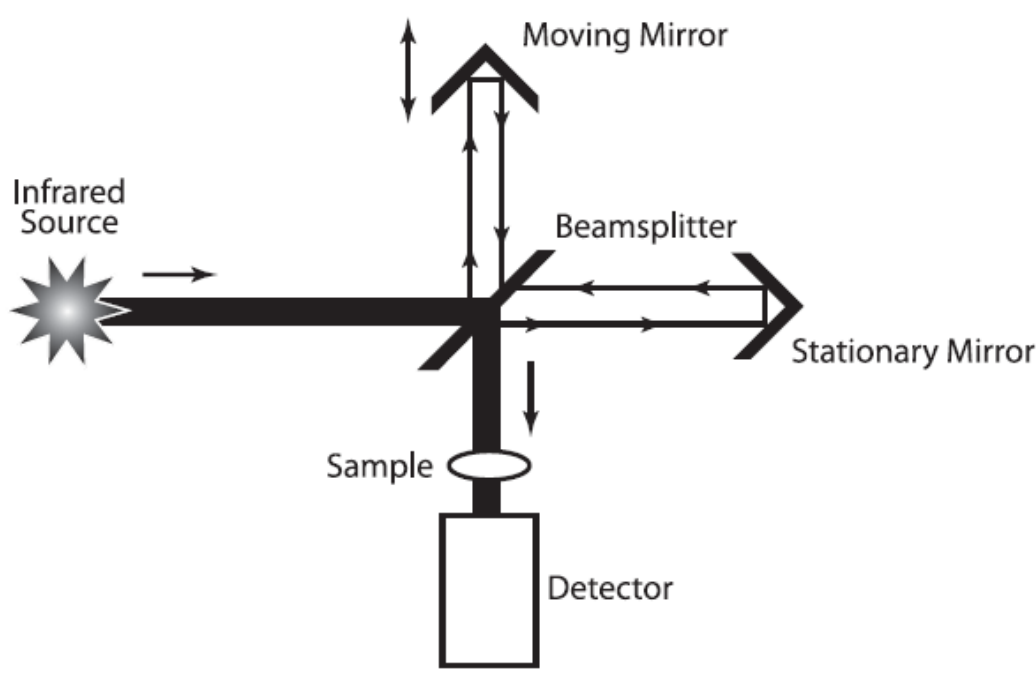

Fig 9. Path of the infrared beam in a Fourier Transform Infrared (FTIR) spectrometer. Adapted from ("FT-IR vs. dispersive infrared " 2002).

\section{II.4. Infrared spectroscopy methods}

Infrared spectroscopy methods are analytical techniques used to obtain the infrared spectra of materials. A good understanding of the available sampling methods is important to any experimental design. In this section we describe two common infrared spectroscopy methods: transmission and reflectance.

\section{II.4.1. Transmission}

Transmission spectroscopy is the most common method used by infrared instrumentation, and it can be performed in liquids, solids and gases. Samples contained inside cells absorb infrared radiation that is transmitted from an infrared emitter through a medium and sensed by a detector in the other side, as illustrated in Fig 10. As mentioned previously, the difference in energy caused by absorption yields information 
about the sample under observation. A principle that is of fundamental importance to quantitative analysis in transmission spectroscopy is Beer-Lambert's Law.

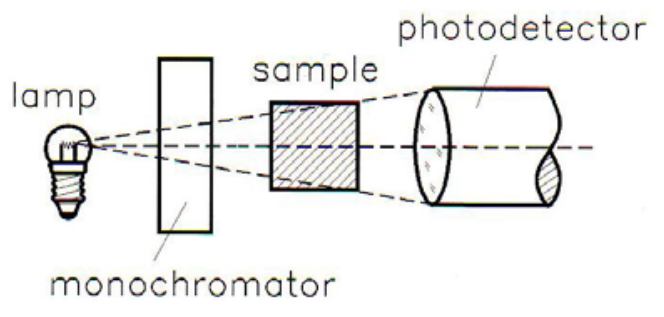

Fig 10. Simple schematic of the absorption by transmission method (Workman Jr., 1998).

\section{II.4.1.1. Beer-Lambert Law}

The Beer-Lambert's Law states that the amount of absorption suffered by an infrared beam of radiation when transmitted through a sample is proportional to the path traveled and the sample's concentration. According to Beer-Lambert's Law, absorption $A$ relates to concentration $c$ and path length $l$ by a molar absorptivity coefficient $\varepsilon$, which is unique to a particular sample, as follows

$$
A=\varepsilon c l
$$

Absorption also relates to intensity $I$ and transmittance $T$ as follows

$$
\begin{gathered}
A=\log I_{o}-\log I=\log \left(I_{o} / I\right) \\
T=I / I_{O} \\
A=-\log T
\end{gathered}
$$

where $I$ and $I_{o}$ are the intensities of the infrared radiation bean before and after being subjected to absorption. As we can observe, absorption is dimensionless and provides quantitative means to derive concentration. 
There are several limitations to Beer-Lambert's Law that can make the empirical relationship between infrared light and absorption become non-linear:

- electrostactic interactions between nearby molecules can cause deviations in absorptivity coefficients at high concentrations

- particulates in the sample, fluoresecence or phosphorescence of the sample can cause the scattering of light

- high analyte concentration can cause changes in refractive index

- concentration changes can cause shifts in chemical equilibria

- $\quad$ stray light

Under certain operating conditions, Beer-Lambert's Law must be modified to accommodate other important properties of the interaction between light and matter. For example, it may be important to consider scattering when calculating the infrared absorption spectrum of micro- and macroscopic cell tissue because of membrane boundaries, or when using infrared spectroscopy in astronomical observations because of clouds or other natural phenomena. Typically, the term scattering is used to collectively describe various physical phenomena which distort a light beam in its geometrically correct light path, possibly changing its wavelength, such as refraction, diffraction, reflection. A detailed discussiong of scattering in spectroscopy is presented elsewhere (Schmidt, 2005). Unless otherwise stated, references to Beer-Lambert's Law will assume it to be in its simple (i.e. linear) and unmodified form. The extent to which these issues apply in our experiments will be discussed in Chapter V. 


\section{II.4.2. Reflectance}

Reflectance spectroscopy methods are also used to obtain the infrared spectrum of solids, gases or liquids, but are specially useful in some instances when normal absorption spectroscopy is not feasible (Schmidt, 2005). Reflectance methods allows for non-invasive, contact-free measurements of solid materials on the surface of complex shaped objects.

Photons that are incident on a material can suffer specular, diffuse or internal reflection. Reflectance spectroscopy concerns itself with the study of infrared radiation that undergoes any of such events. Typically, the type of sample determines what technique of reflectance can be used. In specular reflectance, also known as external reflectance, the instrument can measure infrared light that is reflected from smooth surfaces, as illustrated in Fig 11. Diffuse reflection methods can be used to measure the infrared absorption spectra of powders and rough solids. In diffuse reflection, a sample's surface is illuminated with infrared radiation and the scattered light is collected via appropriate optical components to generate its absorption spectrum. Internal reflection method is the most flexible in terms of sample preparation, and can be used, for instance, to determine the infrared absorption fingerprint of fats. Internal reflection at boundary layers of materials with different refractive indices is accompanied by a small displacement (approximately 1 wavelength) into the thinner medium (Schmidt, 2005). In the presence of a potential absorber, this displacement may cause a small attenuation of the signal. The most widely used technique of internal reflectance is known as attenuated total reflectance (ATR). Total reflectance refers to a method where energy is completely 
reflected when incident at a material at an angle larger than a critical angle. A critical angle is derived from the refractive indices of the materials. In ATR a sample is placed in contact with a crystal, and an infrared radiation beam is aligned in an angle larger than that of the critical angle, such that it undergoes total reflectance. However, a small portion of energy is lost due to absorption by the sample at particular wavelengths. ATR measures the difference in energy caused by the absorption at the sample surface.

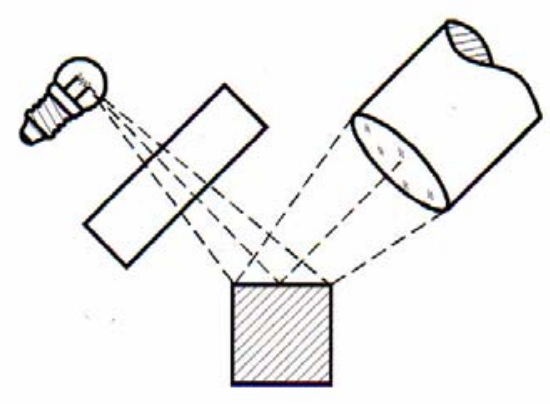

Fig 11. Simple schematic of the spectral reflection method for infrared absorption spectroscopy (Workman Jr., 1998).

\section{II.5. Conclusion}

The objective of this chapter was to introduce the reader to the fundamentals of infrared absorption spectroscopy. We presented a theoretical background into the field, and elucidated the various component technologies (i.e., infrared source, chamber; detector) and infrared spectroscopy methods (i.e., transmission, reflectance) that are important for understanding infrared absorption spectroscope instruments. In the next chapter we present a new infrared absorption spectroscope system, named Infrared Integrated 
System (IRIS), based on LVF optics and 1D array of pyroelectric detectors for the automated detection of gases, vapours and gaseous mixtures. 


\section{CHAPTER III}

\section{INFRARED INTEGRATED SYSTEM}

The Infrared Integrated System (IRIS) that has been developed as part of this thesis is a dispersive analytical spectroscope designed for automated measurements of the infrared absorption spectra of concentrated gases, gas mixtures and vapors, and for the semiautomated measurement of liquids. It is intended to be a low-cost alternative to typical dispersive and non-dispersive infrared spectroscopes, and be capable of functioning in rugged operating conditions with minimal maintenance requirements. In this chapter we discuss the architecture, the system characterization and the performance analysis of IRIS.

\section{III.1. System components}

The system is composed primarily of an infrared source, chopper, gas chamber, infrared detector, valves and a pressure sensor. Fig 12 illustrates a high-level schematic diagram of IRIS. In this section we discuss the details of each component, and its purpose in the overall execution of the system. 


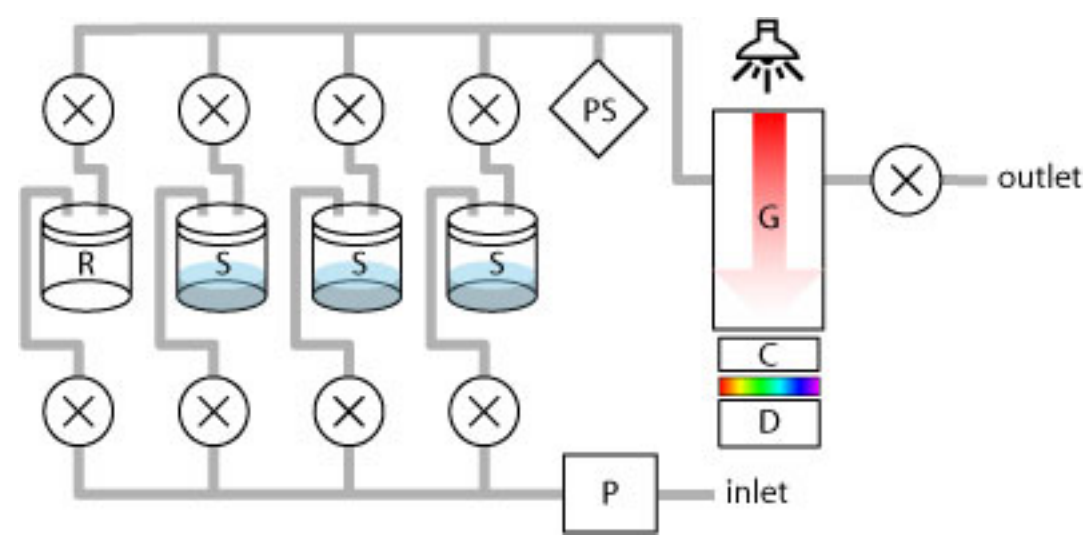

Fig 12. High-level schematic diagram; P: pump, R: reference gas, S: samples, PS: pressure sensor, G: gas cell, C: mechanical chopper, and D: detector.

\section{III.1.1. Detector}

The infrared detector combines a linear variable filter (LVF) and an array of 64 pyroelectric detectors; it can be purchased from IR Microsystems (www.irmicrosystems.com). As illustrated in Fig 13, the LVF is a wedge-shaped optical filter that functions as a wavelength dispersive optical component, allowing certain wavelengths to pass through at certain positions in a linear fashion. Due to optical limitations, a manufactured LVF can only range from an initial wavelength to twice that wavelength; in the case of IRIS, the LVF covers the range 5.27-10.5 $\mu$ m (i.e. 1898-955 $\mathrm{cm}^{-1}$ ), which spans a large portion of the mid infrared fingerprint and functional regions. The LVF sits atop a 64-pixel pyroelectric detector array, which has a surface area of 14.7 $\mathrm{x} 3.5 \mathrm{~mm}^{2}$ and pixel pitch of $0.2 \mathrm{~mm}$ (interlaced). Each pixel in the array extends an area of $0.12 \times 1 \mathrm{~mm}^{2}$. 

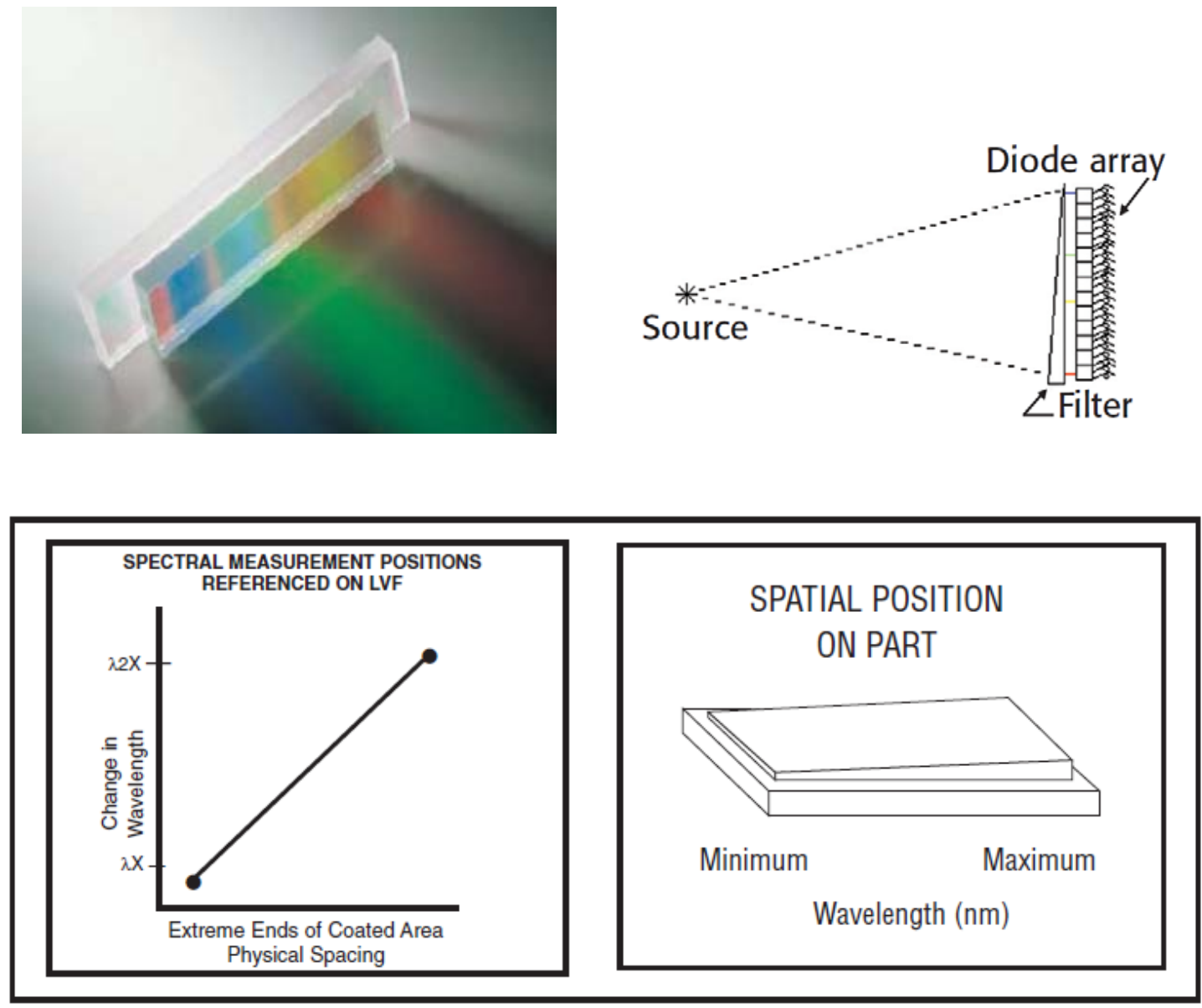

Fig 13. Top-left: Photograph of an LVF. Top-right: a diagram of the infrared radiation path as it passes through the LVF and reaches a detector array. Bottom-left: a display of the linear wavelength output characteristic of the LVF. Bottom-right: rendering of the LVF's physical aspects (JDSU, 2006).

The detector offers a direct PC connection through a RS232 port, and comes with a device driver for rapid integration with National Instruments' Labview software. The device driver consists of a so-called virtual instrument (vi) module of the 64-channel parallel readout, which can be polled in synchronization with the chopper via software configuration directives. 


\section{III.1.2. Source and chopper}

The infrared source is an $11 \mathrm{~W}$ thermal emitter supplied by Boston Electronics (model IR-12K). This source has $80 \%$ emissivity in the infrared region, and is typically operated at $825 \mathrm{C}^{\circ}$ temperature with a $5 \mathrm{~V}$-power supply. As shown in Fig 14 , the coil is mounted horizontally on a cylindrical alumina substrate, and is suited for use with a parabolic mirror. In IRIS, a parabolic mirror is necessary to obtain collimated infrared beams at the LVF; collimation affects the resolution power of the system, as discussed later in section III.3.4. In a nutshell, the more perpendicular the incident infrared light is with respect to the LVF surface, the higher the capability of IRIS to discriminate absorption at different, yet close wavenumbers.

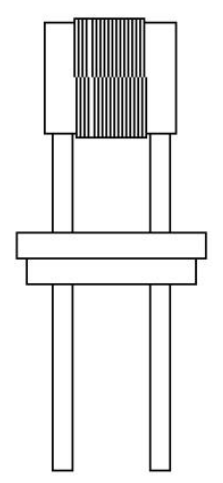

Fig 14. Schematic of the infrared source (model IR-12K from Boston Electronics).

Pyroelectric detectors require a modulated signal for correct operation, which can be accomplished by means of chopper or a trigger. The trigger transforms the emitter power supply from a DC into an AC power supply, and is typically modulated by an 
external clock frequency. In contrast, the chopper is a mechanical device consisting of a rigid and flat vane mounted on a motor that rotates with a limited angle, opening and closing the light path with a given frequency. The movement of the blade follows the direction of the current flow in the motor winding. Thus, alternating the current causes the blade to open and close the chopper at a frequency range from DC to $50 \mathrm{~Hz}$. In order to avoid jitter, IRIS employs a chopper for signal modulation. Another advantage of a chopper based system is that it enables the use of sources with high thermal mass for high wattage. In the IRIS configuration, the chopper is operated by a driver, which provides a $10 \mathrm{~Hz}$ TTL signal, and allows the chopper to have a frequency stability of 0.005\% (IR Microsystems, 2004).

\section{III.1.3. Chamber and windows}

IRIS uses a gas cell from Buck Scientific (model Beta Gas Cell) as the gas chamber. The gas cell is $100 \mathrm{~mm}$ long and $25 \mathrm{~mm}$ in diameter. Shown in Fig 15, the gas cell has a Pyrex body and two Pyrex filling stems that are used as inlet and outlet in the gas circuit. Two zinc selenide $(\mathrm{ZnSe})$ windows $(25 \mathrm{~mm}$ diameter, $4 \mathrm{~mm}$ thickness) were special ordered for the ends of the gas cell (Cradley Crystals Corporation; Nizhni Novgorod, Russia); though significantly more expensive than other materials (e.g., $\mathrm{NaCl}, \mathrm{KBr}$ ), $\mathrm{ZnSe}$ can withstand exposure to most non-acidic solvents, and has low absorption in the mid infrared region, making it ideal for our purposes. A leak-tight assembly, capable of withstanding 15-psig pressure, was achieved by using $1 \mathrm{~mm}$-thick rubber washers between each ZnSe window and the Pyrex body. 


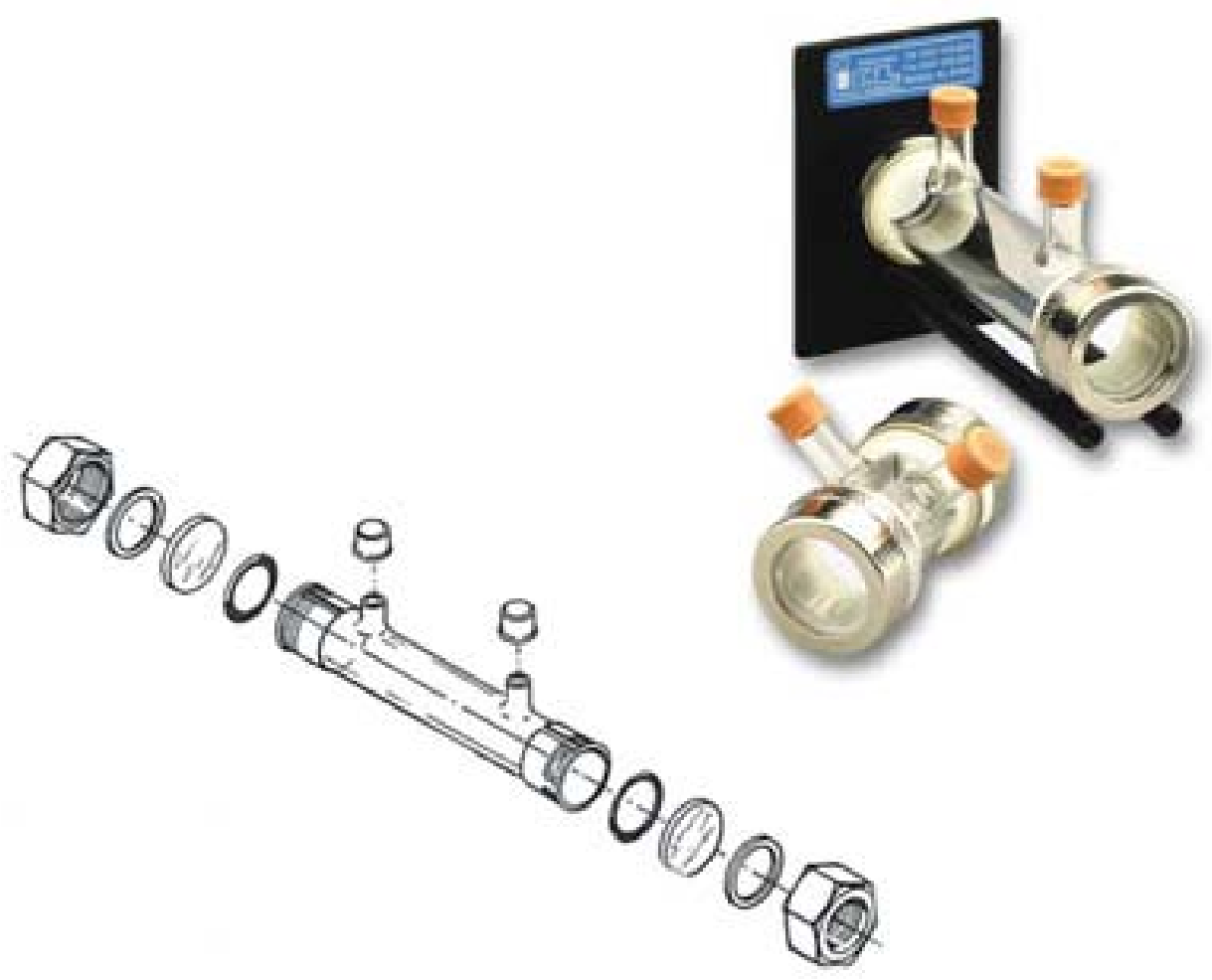

Fig 15. Top: photograph of two gas cells of different lengths. Bottom: a gas cell's component arrangement (model Beta Gas Cell from Buck Scientific).

\section{III.1.4. Pump, valves, samples and other components}

IRIS uses an oil-less diaphragm pump from GAST (model 10D1125-101-1052). This pump features a DC motor capable of 15-psig maximum pressure. IRIS employs the pump in pressure mode in order to pressurize the gas chamber which leads to improvement in signal response for low-concentrated gases.

The valves used are from Clippard (model EC-2M-12). These valves have a fast response time, and operate in a quiet fashion. The valves' moving parts travels less than 0.007 inches, and sustain pressures up to $100 \mathrm{psi}$ to ensure a secure seal. The valves are mounted onto 4-channel manifolds, as shown in Fig 16. 


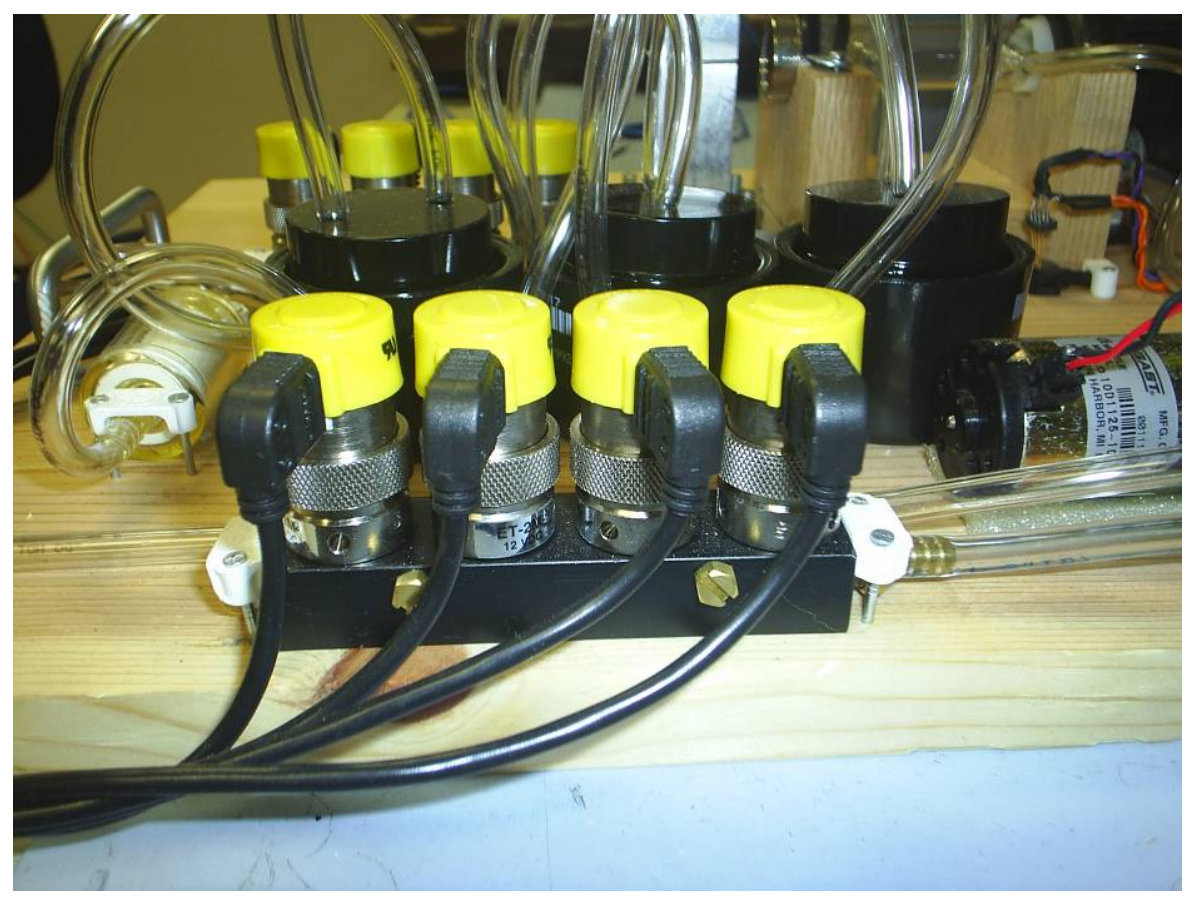

Fig 16. Valves mounted onto a 4-channel manifold.

As illustrated also in Fig 17, samples are placed inside 200ml glass containers, which are enclosed with lids that have inlet and outlet openings. A separate sample container is destined for a gas filter that is used in the system for the measurement of reference gases. 


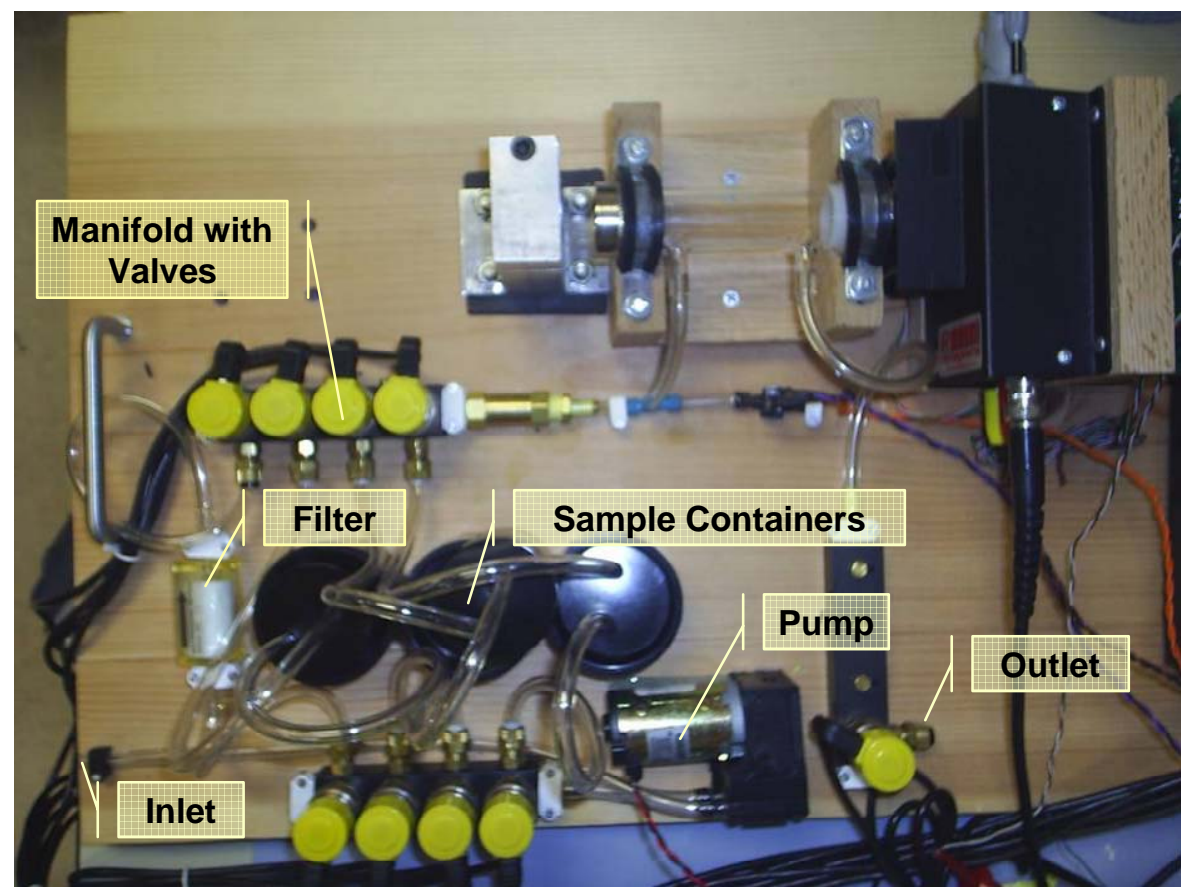

Fig 17. Close-up view of the gas circuit in IRIS.

\section{III.1.5. Electronic circuitry}

The electronic circuits designed to drive all the IRIS components are described in this section. In essence, a host computer controls all operations in IRIS through a data acquisition card (DAQ) from National Instruments (model USB-6009). Two analog input channels in the DAQ are utilized to read the amplified pressure sensor signal, and six digital output channels are utilized for controlling the pump and valves. As discussed earlier, data from the LVF are read in through an RS-232 interface.

To measure pressure inside the gas chamber we used a differential pressure sensor (Honeywell model 24PCCFA6D). This piezoresistive device has two ports for differential sensing. In IRIS, one of the ports is connected to the gas circuit and the other 
is left open, such that the sensor provides gauge measurements with ambient pressure as the reference. The pressure sensor provides a stable $\mathrm{mV}$ output for the $0.5-250 \mathrm{psi}$ range, with a linear relationship of $15 \mathrm{mV}$ per psi. As mentioned earlier, the maximum pressure provided by the pump is 15 -psi, which is well in the range of the pressure sensor. The $\mathrm{DAQ}$, however, is unable to operate in the $\mathrm{mV}$ range, so an amplification circuit, illustrated in Fig 18, is required. IRIS utilizes the pump, valves and the output of the pressure sensor to pressurize the gas chamber to a desired level with precision.

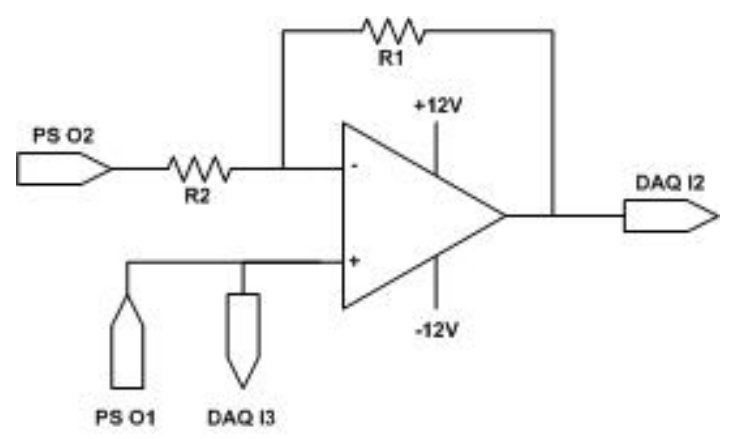

Fig 18. Pressure sensor signal amplification circuit. PS 01 and PS 02 are the differential pressure sensor output. The values of the resistors R1 and R2 are $1 \mathrm{~K} \Omega$ and $100 \mathrm{~K} \Omega$, respectively. DAQ I2 and DAQ I3 are the amplified differential input to the DAQ. The operation amplifier is a standard 741.

A driver circuit is also used to control the valves, as illustrated in Fig 19. Except for the exit valve downstream from the gas cell, all valves in IRIS are used in pairs to seal the inlet and outlet of each sample container (refer to Fig 12). In the case of the exit valve, $\mathrm{V} 2$ in the driver circuit is not connected. 


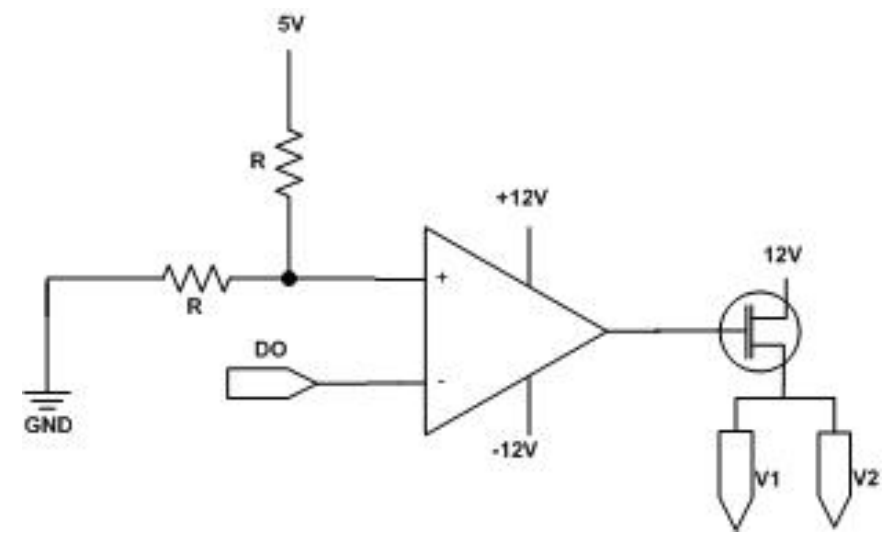

Fig 19. Valve driver circuit. DO is the DAQ output control for the valves. V1 and V2 are the output of the circuit. Note that V1 and V2 are operated simultaneously. The operation amplifier used is a 741; the MOSFET is an ECG261.

\section{III.2. Configuration}

The IRIS configuration enables a completely automated method of measuring the infrared absorption spectrum of gases or gas mixtures. Fig 20 illustrates a detailed diagram of the IRIS setup. The air pump is placed upstream from the sample containers, and used to provide air flow to or pressurize the gas cell. A maximum pressure of 15psig can be achieved in the gas chamber. IRIS allocates one inlet channel to a dry reference gas (filtered room air). Liquid or solid samples can be placed in any of three different sample containers, which are individually enclosed by a pair of valves. By enclosing the containers, contamination between samples is minimized. The gas in the headspace of the sample container is pumped to the gas chamber, where it is typically pressurized. The infrared source is powered when the system is turned on, and provides the infrared radiation necessary for the measurement of the infrared absorption spectra of the pressurized gas in the gas cell. As with the source, the chopper and the detector are 
also powered when the system initializes. The detector automatically takes one measurement every second, and transmits the data through the 64-channel parallel readout circuit and serial port to the computer.

IRIS can be operated by the user through a graphical user interface, as illustrated in Fig 21. There are 3 modes of operation: manual, automated and scripted. A user can manually save and load data from a reference gas to obtain an infrared absorption spectrum of a sample. The interface allows the user to manually control the pump and any of the valves with the click of a button. In the automated measurement mode, complete measurement cycle processes are prerecorded, and can be played from a pulldown menu. Examples of such processes are system cleaning or individual sample measurements. Scripted execution mode provides the user with the ability to execute complex sequence of events from a spreadsheet file (CSV format), thus allowing for enormous flexibility in experimental design.

There are two configuration parameters of interest to the operation of IRIS: phase angle and integration time. The phase angle determines the phase offset between the chopper period and the polling of data from the detector array. Thus, by adjusting the phase angle parameter, the user can set the detector to poll data in precise synchronization with the chopper. The integration time parameter allows the user to specify the number of periods in which measurements are averaged. This is particularly useful when attempting to identify gases close to the detection limits of the instrument. All things being equal, longer integration time yields more precise measurements as a result of averaging out uncorrelated noise. 

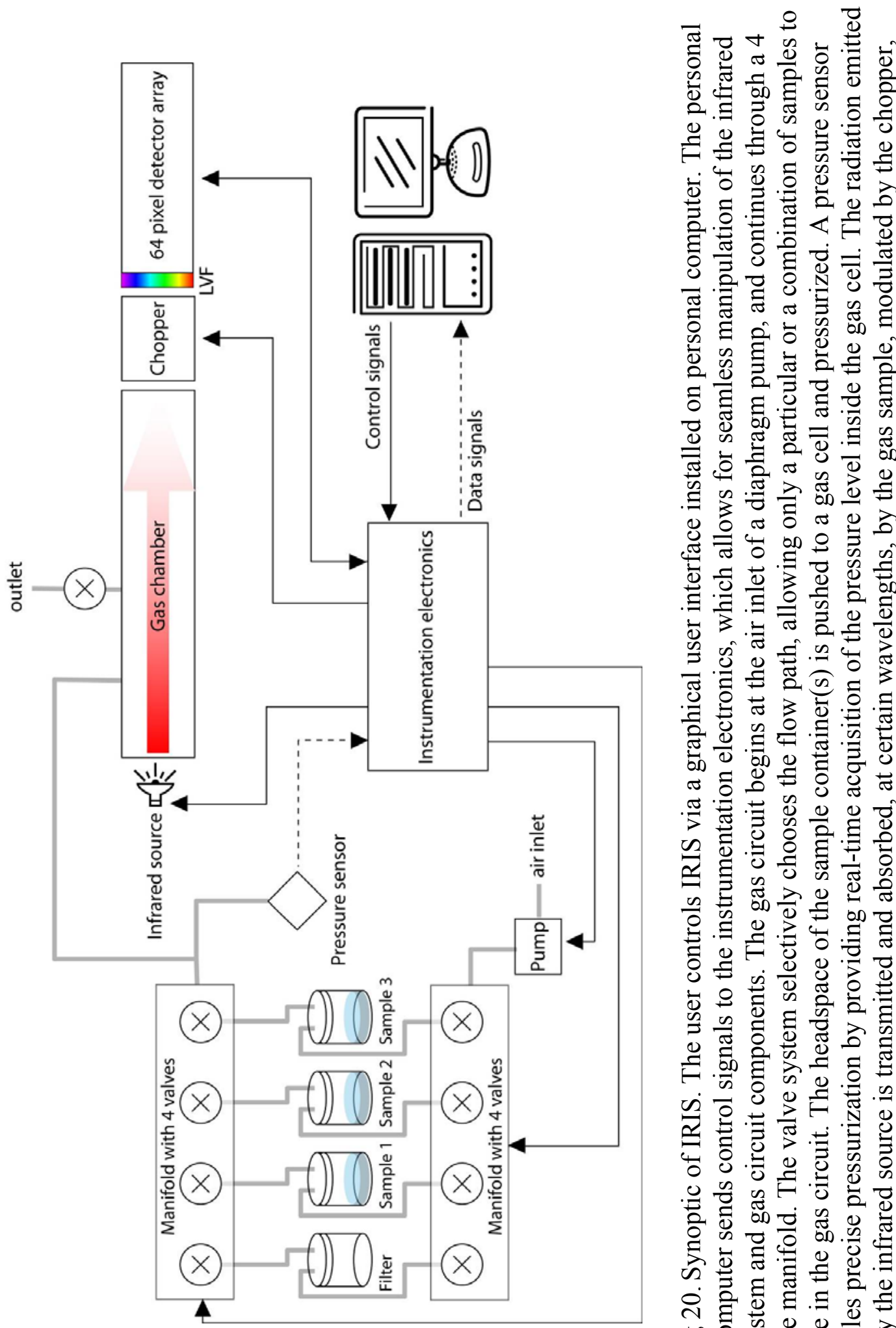

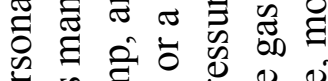

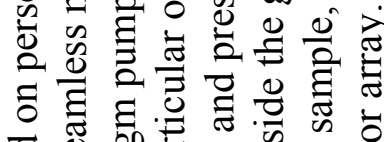

ठ্

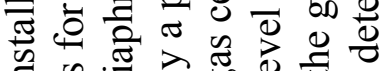

.

远先

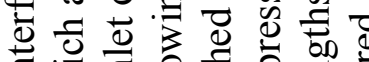

.$\Xi \Xi$.

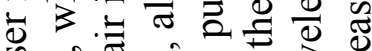

की बै ही.

శึ

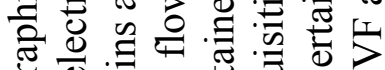

के

펵

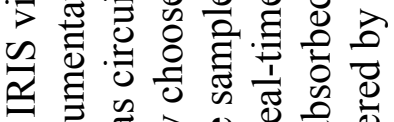

可

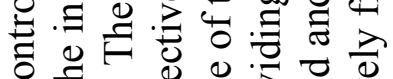

$\delta \doteq \dot{0} \frac{0}{0}$ ठ

ธ。

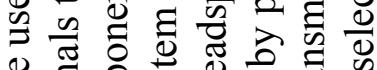

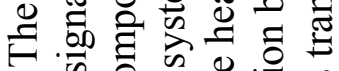

न क क त क 0 .

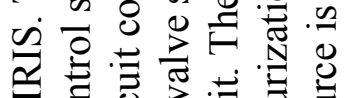

廿

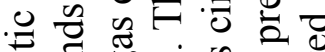

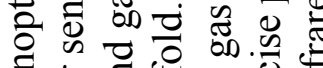

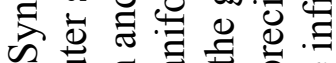

ণ

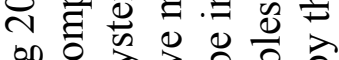

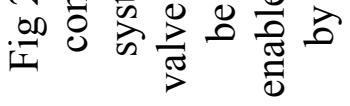




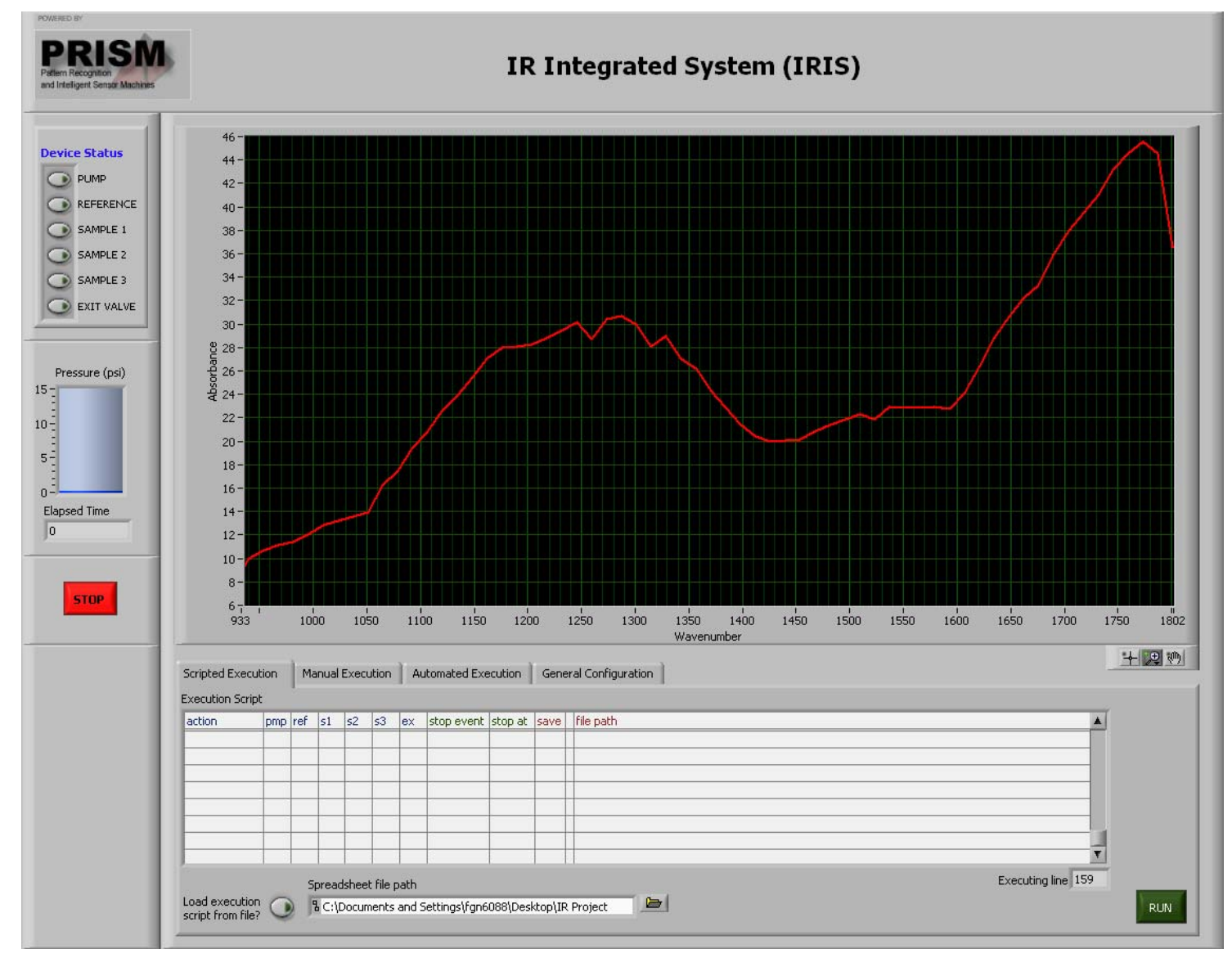

Fig 21. IRIS software interface. On the left, the device status panel displays the state of the pump and valves, and allows their manual control. The pressure indicator displays real-time information about the pressure level inside the gas chamber. On the top-center, the virtual spectroscope displays the intensity of the signal captured at each wavelength in the detector array. On the bottom-center, the tabbed panel allows for rapid access to the three execution modes available (i.e. scripted execution, manual execution, automated execution) and to the configuration settings.

\section{III.3. Characterization}

In order to determine whether IRIS can be used in a specific laboratory or field application, it is necessary to understand its capabilities. Knowledge about the system's limitations is also required prior to experimental planning, analytical process or protocol 
design. In addition, it is important to provide the means to evaluate the level of confidence of a measurement for risk assessment, and to make the user aware that in some cases (e.g. when near detection thresholds) it may be necessary to have an independent measurement technique for validation. In this section, we provide the system characterization by evaluating a number performance metrics; uncovering the detection limits and resolving power of IRIS.

\section{III.3.1. Performance metrics and analysis}

In this section we present a number of performance metrics used to evaluate gas detection systems (Chou, 1999), and analyze IRIS based on them. These metrics can be used to establish qualitatively or quantitatively the performance of most gas detection technologies.

\section{III.3.1.1. Accuracy, precision and repeatability}

Accuracy is the correctness of a measurement, which is assessed by comparing the measurement with the ground truth or an accepted value. Currently, there is no standard in which to compare gas detection systems. Thus, the term accuracy is often widely but inappropriately used. Precision is a quantifiable measure that indicates the system's ability to produce repeatable results. In the context of chemical detection systems, repeatability refers to the systems' ability to provide similar measurements of concentrations when subjected to exactly calibrated gas samples. A system can therefore be precise but not necessarily accurate, since accuracy can only be determined when compared to a standard (Chou, 1999). We will limit our evaluation in terms of the 
capability of the system to obtain consistent and precise measurements, and compare it to high-resolution FTIR spectra from the online database at the National Institute of Standards and Technology's (NIST) Chemistry WebBook (Linstrom \& Mallard, 2003).

Another important metric that relates to precision is uncertainty. Uncertainty indicates the reliability of a single measurement output. Precision errors are typically interpreted as zero-mean random variables whose uncertainties can be reduced by replication (Tripp \& Tcheng, 1999). There can be many sources of uncertainties within an instrument, and they can be jointly represented as a combined standard uncertainty $u_{c}$. In IRIS, there are three main sources of uncertainty: fluctuation in emissivity by the source, noise at the detector, and inexact pressurization of the gas cell. Since fluctuation in emissivity by the source and in readings at the detector cannot be individually examined in our system, we investigate raw signals to derive the uncertainty that results from the joint effect of these sources.

For this purpose, raw signals were sampled every second, for approximately 15 hours. We estimated the raw signal's expanded uncertainty by calculating the positive square root of the raw signal variance multiplied by a coverage factor of 2 . This type of uncertainty estimate is consistent with NIST's guidelines for Type B evaluation of uncertainty from experimental data (Taylor \& Kuyatt, 1994). Fig 22 illustrates the system response at every second for approximately 15 hours after the device's required warm up period. Fig 23 shows the relative uncertainty, which was obtained by dividing uncertainty by the mean of the measurements. The average relative expanded uncertainty of the raw signal measurements is estimated to be $8.36 \%$. In addition, we investigate the 
uncertainty due to inexact pressurization at the chamber by performing similar procedures (i.e. Type B evaluation) for raw signals acquired from 480 same-pressureconfiguration measurements. The resulting expanded uncertainty due to inexact pressurization is estimated to be $2.76 \%$. Therefore IRIS's combined expanded uncertainty with a coverage factor of 2 is estimated to be $11.12 \%$. By analyzing the NIST Quantitative Infrared Database, we can see that on average their high resolution instrumentation and methods showed an expanded uncertainty estimate of 2.2\%. Therefore, IRIS represents a considerable precision tradeoff when compared to the instrument used to obtain the NIST Quantitative Infrared Database.

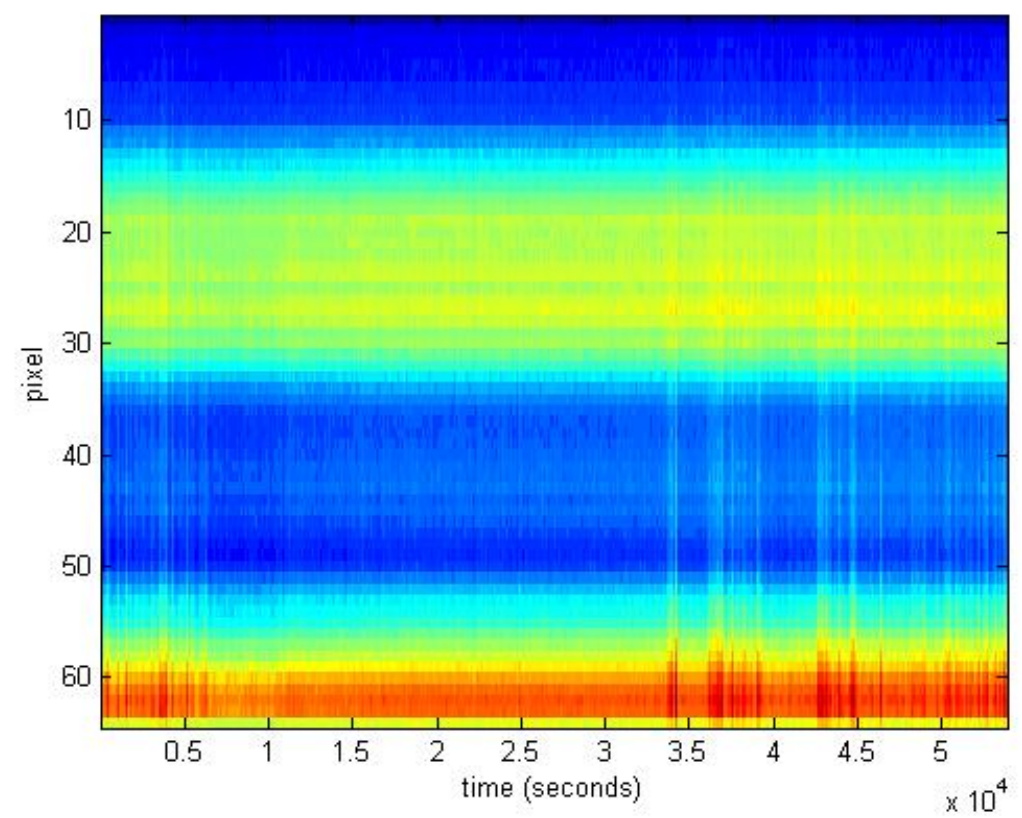

Fig 22. Raw signal measurements over an approximately 15 -hour period. Random levels of noise, represented as sudden changes in detected intensity, can be observed. 


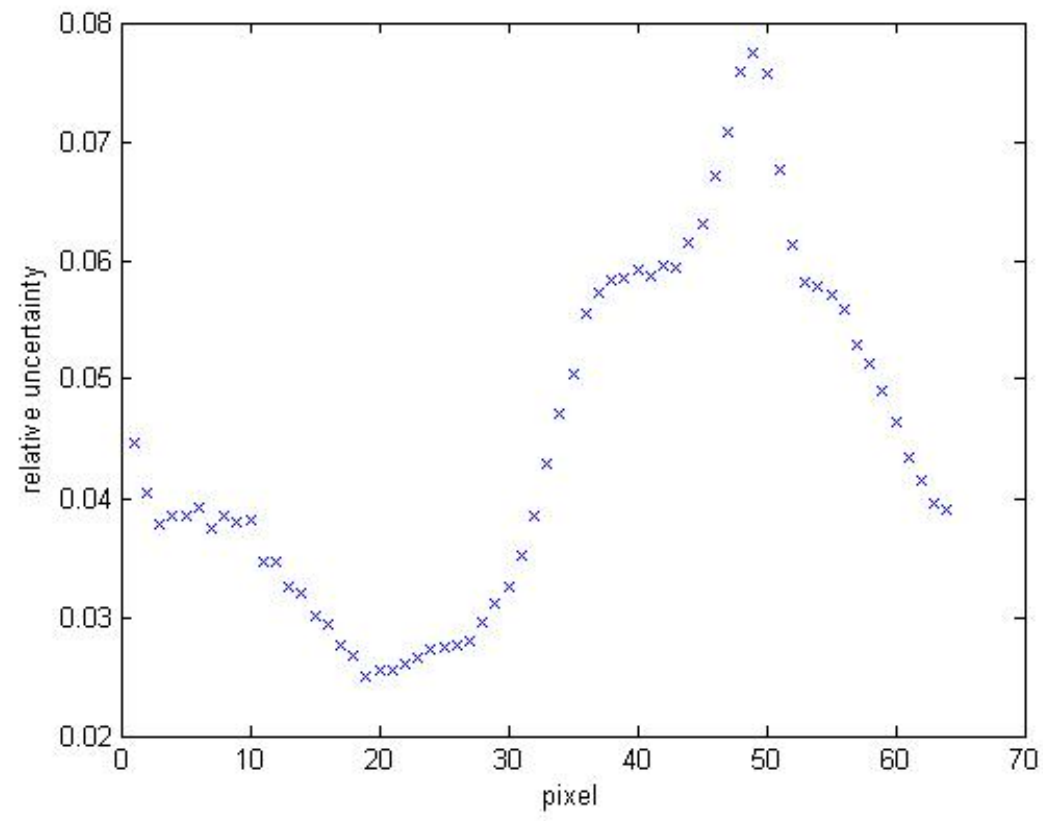

Fig 23. Relative uncertainty by pixel, calculated by dividing the expanded uncertainty by the mean of the measurements.

\section{III.3.1.2. Linearity}

Linearity refers to the extent to which the output signal of the system changes linearly when exposed to linear changes in the concentration of the sample. In order to achieve linearity, most infrared detectors need to be calibrated. It is important to notice that measurements of absorption derived from Beer-Lambert's law are restricted to a range of acceptable concentrations. For instance, when the concentration level is close to saturation, the calculated transmittance is extremely low causing unacceptably high absorption measurements. Typically, Beer-Lambert law's useful (i.e. linear) concentration range results in absorption responses from 0.15 to 0.85 (arbitrary units). 


\section{III.3.1.3. Specificity or selectivity}

The specificity or selectivity of a gas detector device indicates its ability to detect a target gas without being affected by the presence of other interfering gases. Infrared absorption spectroscopes for gas detection are among the devices with least specificity properties. In particular, IRIS will respond to any heteronuclear diatomic or polyatomic molecule in the infrared radiation path that causes sensible absorption in the 5.27$10.5 \mu \mathrm{m}$ wavelength range. Nonetheless, infrared absorption systems can accurately target specific gases by using signal processing techniques if sufficient knowledge about the matrix is available (See section IV.4).

\section{III.3.1.4. Interferences}

Gas detectors are not selective to a single gas. In terms of infrared absorption spectroscopes, when absorption bands of chemicals in a mixture overlap, they are reflected in an additive manner. Albeit chemicals have unique infrared absorption spectra, portions of spectrum may be very similar. This can be exemplified when analyzing the functional region of the spectrum of certain chemicals that belong to the same functional group. In this scenario, the absorption spectrum of the interfering gas can appear simply as an additive factor to the chemical under observation. IRIS's wavelength range comprises of only a portion of the functional and fingerprint regions. If the interfering gas have undistinguishable features in the fingerprint as well, it becomes impossible to separate the interfering gas from the measurand. In addition, limitations in resolution may also mask spectral differences in chemical mixtures. For 
this reason, it is recommended that the operator of IRIS have some prior knowledge of the mixture before performing analytical measurements.

\section{III.3.1.5. Response/recovery time}

Response/Recovery time indicates the time needed for a sensor to read a percentage of the full-scale after being exposed to full-scale concentration. For instance, $R_{90}=30 \mathrm{~s}$ indicates that $90 \%$ of the full-scale can be read in 30 seconds after exposure to saturation concentration. In infrared absorption spectroscopes, the measurand is typically contained in a remote gas cell, which is not in direct contact with the sensor. Since measurement is performed remotely, the response/recovery time is only limited by the sampling rate of the detector. In terms of response/recovery time, infrared detector technologies are generally much superior to chemical sensor technologies, whose time constants are typically one or two orders of magnitude larger, particularly in the case of recovery times. The transient response of the gas or detector array to the infrared radiation are orders of magnitude shorter than the sampling time, and can be neglected. Therefore, a steady-state response is reached in IRIS within one sampling period ( $1 \mathrm{sec})$; thus $\mathrm{R}_{100}=1 \mathrm{~s}$, which is in pair with the scan time required by most FTIR instruments on the market today.

\section{III.3.2. Detection limits and sensitivity}

Two metrics are commonly used to convey information about a device's detection limits: limit of quantitation (LOQ) and limit of detection (LOD). LOQ is defined as the lowest concentration of an analyte in a sample that can be determined with acceptable 
precision and accuracy under stated operational conditions. LOD is defined by the International Union of Pure and Applied Chemistry (IUPAC) as follows (Thomsen et al., 2003):

The limit of detection, expressed as concentration, $c_{\mathrm{L}}$, or the quantity, $q_{\mathrm{L}}$, is derived from the smallest measure, $x_{\mathrm{L}}$, that can be detected with reasonable certainty for a given analytical procedure. The value of $x_{\mathrm{L}}$ is given by the equation $x_{\mathrm{L}}=x_{\mathrm{bi}}+k s_{\mathrm{bi}}$ where $x_{\mathrm{bi}}$ is the mean of the blank measures, $s_{\mathrm{bi}}$ is the standard deviation of the blank measures, and $k$ is a numerical factor chosen according to the confidence level desired.

In general, the numerical factor is chosen as $\mathrm{k}=3$ to give a level of confidence of approximately $90 \%$. In practice, LOD is typically determined by following one of two approaches: method of detection limit (MDL) or instrument detection limit (IDL). MDL refers to the lowest concentration of analyte in blank or sample that a method can detect reliably, whereas IDL is defined as the smallest signal above background noise that an instrument can detect reliably. Fundamentally, MDL and IDL differ in the sampling method. In MDL we examine a spiked solution with a small concentration of an analyte (approximately $5 \%$ above noise level) in a sample matrix, whereas in IDL we examine a blank (e.g. reagent water). In other words, IDL considers clean matrices, whereas MDL considers real-life matrices. Therefore, MDL is typically found to be more realistic but 2-5 times worse than IDL. LOD indicates the level at which we can distinguish a signal from the background noise, but does not necessarily indicate the smallest concentration that can be measured. It is agreed that, in order to measure concentration levels, the solution must yield a signal that is 10 standard deviations from the blank (Thomsen et 
al., 2003). Thus LOQ $=10 \times s_{\mathrm{bi}}=3.3$ LOD. Fig 24 shows the IRIS detection limits obtained by using IDL and tap water as the blank.

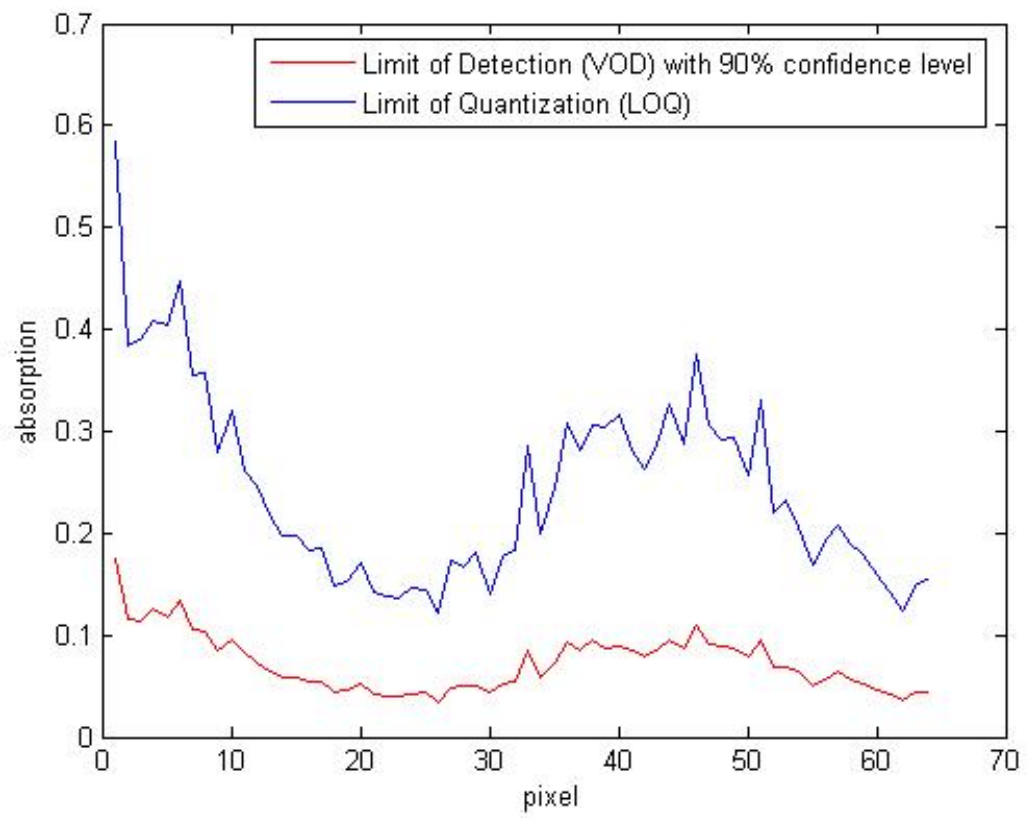

Fig 24. IRIS absorption detection limits.

In our case, because of the unavailability of an instrument (e.g. photoionization detector) to measure concentrations or a chemical process (e.g. gravimetric) to generate a gas with precise sample concentration, and because gas measurements in IRIS are obtained from volatile-organic-compounds (VOC) of liquid samples inside a sample compartment delivered to the gas cell, we will discuss sensitivity in terms of the concentration of the sample in the liquid phase. Sensitivity to a particular chemical depends on its concentration in a matrix and its extinction (i.e. absorptivity) coefficient profile. To exemplify this concept, consider the absorption spectra of acetone in water 
matrix at 1:100 dilution - using water in a separate sample compartment to acquire a reference gas - as illustrated in Fig 25. This solution was chosen because of acetone's high evaporation rate (i.e. 7.7 w.r.t. n-Butyl Acetate) and defined absorption peaks in the LVF region. The solution has a total volume of $100 \mathrm{ml}$, and is placed in a cylinder shaped glass compartment ( $4.4 \mathrm{~cm}$ in height by $4.4 \mathrm{~cm}$ in diameter).

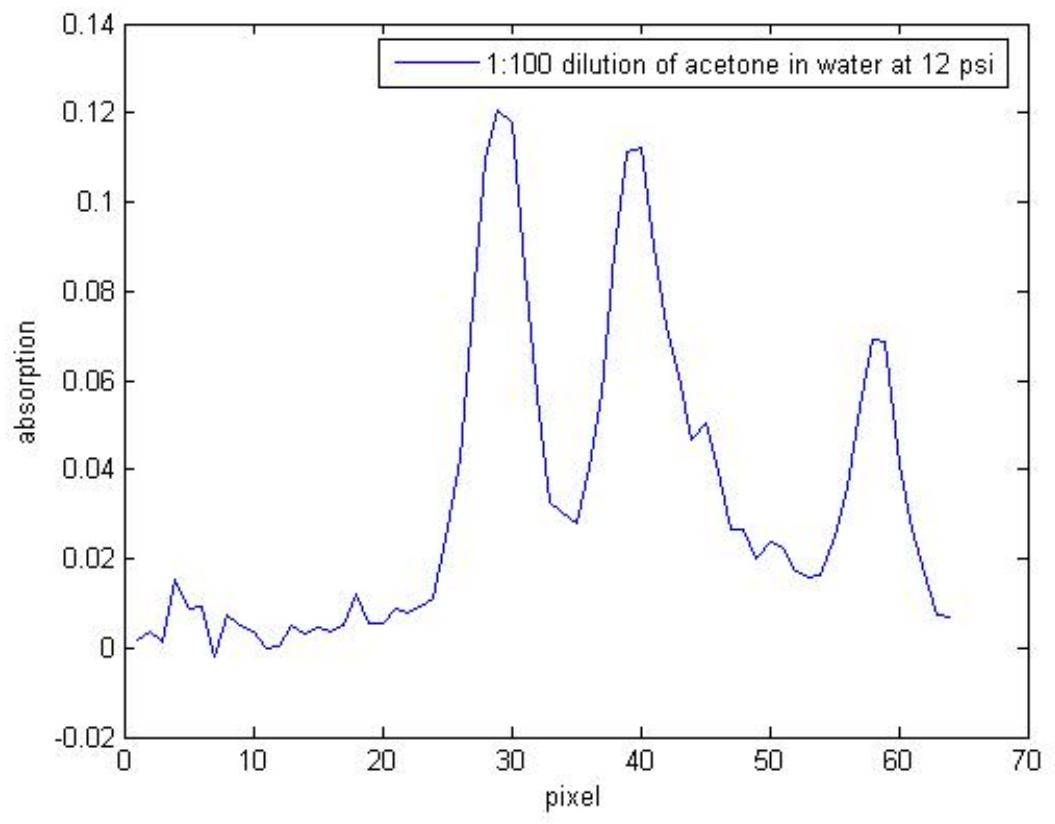

Fig 25. Average of 47 measurements of the infrared absorption spectra of VOC components for 1:100 dilution of acetone in water at 12 psi. Notice that, in order to obtain just the peaks that are due to acetone, we have taken water vapor as a reference spectrum.

In order to express detection limits $x_{\mathrm{L}}$ in terms of concentration, we use sensitivity $S$, which is the slope of the calibration curve, i.e., $S=$ $\Delta$ concentration/ $\Delta$ absorption

$$
\mathrm{c}_{\mathrm{L}}=k \times s_{b i} \times S
$$


Using the largest response (pixel 29), the sensitivity for the liquid solution above is estimated as:

$$
S=(1 \%-0 \%) /(0.1204 \mathrm{abs}-0)=8.3056 \% / \mathrm{abs}
$$

which, using $\mathrm{S}_{\mathrm{bi}}=0.0186$ (sample standard deviation of the blank samples), yields a detection limit for acetone (in water matrix) of:

$$
\mathrm{c}_{\mathrm{L}}=3 \times s_{b i} \times S=3 \times 0.0186 \times 8.3056=0.46 \%
$$

\section{III.3.3. Resolving power}

Resolving power $R$ is a metric used to compare different spectrometers, and is defined as $\lambda / \delta \lambda$, where lambda is the wavelength range, and $\delta \lambda$ is the smallest wavelength interval that can be resolved (Passerini et al., 2003). Generally, this interval is determined by the limitation imposed from a specific component in the system. In IRIS, the two elements that impose the most limitations are the LVF filter and the number of pixels in the detector array. The LVF manufacturer reports that the filter has a resolution of $1.5 \%$ of the transmitted wavelength, when the illuminating beam is collimated and perpendicular to the filter plane (Passerini et al., 2003). The resolution power of the LVF is therefore $R=\lambda / \delta \lambda=\lambda /(1.5 \times \lambda)>66$. However, employing an LVF with a larger resolving power would not improve the resolving power of IRIS, since the detector array imposes the main limitation to the system (i.e. $R=64$ ). It is important to note that LVFs with much larger resolving powers (e.g. $R=20000$ ) have been reported (JDSU, 2006), which would warrant the use of larger (e.g. 128-pixel) detector arrays. 


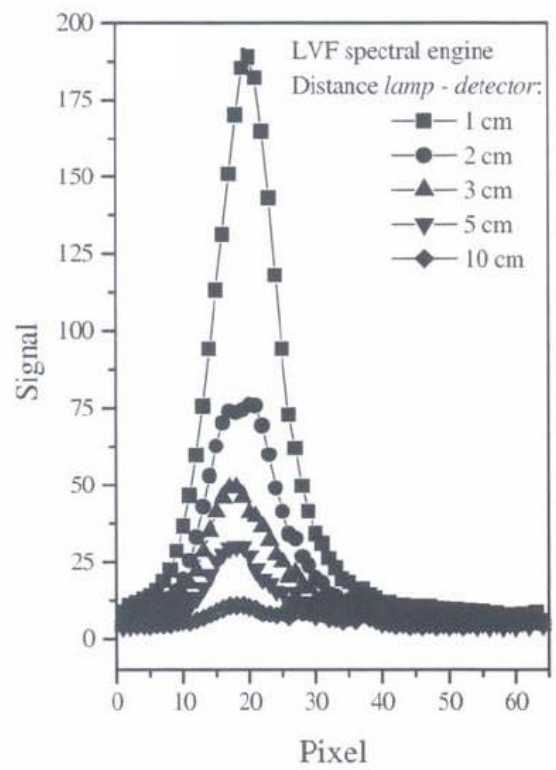

Fig 26. Signal intensity as a factor of source-detector distance. Adapted from Passerini et al., 2003.

\section{III.3.4. Resolution and intensity losses}

Placement of the paraboloidal reflector plays an important role in the resolution of IRIS. As stated in (Passerini et al., 2003), resolution is dependent on the quality of light collimation. The ability of the system to deliver collimated infrared beams that are perpendicular to the detector depends not only on the quality of the paraboloidal reflector, but also on the distance between the paraboloidal reflector and the LVF. Fig 26 shows the relationship between signal intensity and source-detector distance, when a $3.46 \mu \mathrm{m}$ filter is introduced on the light path. Notice that the tradeoff between intensity and distance is also reflected in the signal's full-width at half-maximum (FWHM), which can be used as an indicator of resolution. For instance, at a $2 \mathrm{~cm}$ distance from the detector, the paraboloidal reflector poses the FWHM across more than 4 pixels, resulting 
in a resolution of $0.096 \mu \mathrm{m}$ and resolving power $\mathrm{R}=36$ (Passerini et al., 2003). Although moving the source away from the detector may improve resolution, ultimately, improvement is bound by the resolution power of IRIS (i.e. $R=64$ ).

\section{III.3.5. Steady state}

IRIS reaches a steady delivery state at approximately 20 minutes after it is powered. Fig 27 shows the response of each pixel with respect to time after initial power-up as a false color image.

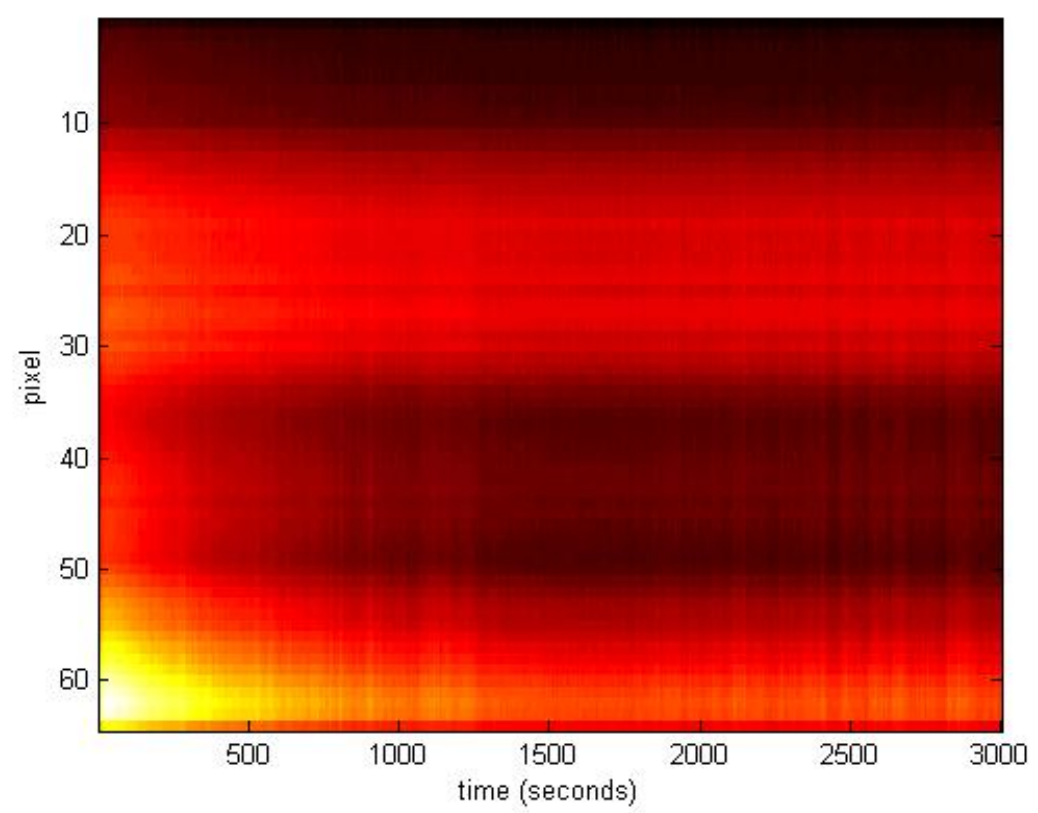

Fig 27. False color image of raw signal response after initial power-up.

Notice that the intensity at most pixels is higher initially than after it reaches stability. This can be explained by principles of self-emission (Couture, 2001): although detector materials are designed to have low absorption, and low corresponding emission, the emissivity of all materials is a function of temperature and wavelength. As the 
temperature at the detector rises because of exposure to the infrared source, so does its self-emission. As a result, a decrease in the AC amplitude of the signal generated by source and chopper can be observed. Furthermore, during this initialization period the source reaches its final and stable operating temperature (i.e. $850 \mathrm{C}^{\circ}$ ).

\section{III.4. Conclusion}

Recent advances in pyroelectric detector array and LVF technologies have inspired the development of IRIS (Infrared Integrated System). IRIS is a low-cost spectroscope designed for the measurement of infrared absorption spectra in gases, vapours and gas mixtures in the mid infrared region. Though a bench-top configuration at its present stage, the prototype can be easily enhanced to yield a compact and ruggedized instrument. In this chapter, we have explained the system configuration details, and discussed the system characteristics in terms of performance metrics commonly used to describe gas detection systems. 


\section{CHAPTER IV}

\section{SPECTRAL SIGNAL PROCESSING}

The objective of the spectral signal processing techniques presented in this chapter is to assist with the detection, identification and concentration analysis of chemicals present in a sample. The class of signal processing problems that involves characterization of the input signal is referred to as signal interpretation (Oppenheim \& Schafer, 1996). In infrared absorption spectroscopy, signal interpretation for the purpose of concentration analysis is referred to as calibration, which can be simply viewed as a multivariate regression problem. Detection and identification are signal interpretation problems that can be solved through pattern recognition. As illustrated in Fig 28, there are typically five stages in a pattern recognition system: data acquisition, preprocessing, feature selection, classification and validation.

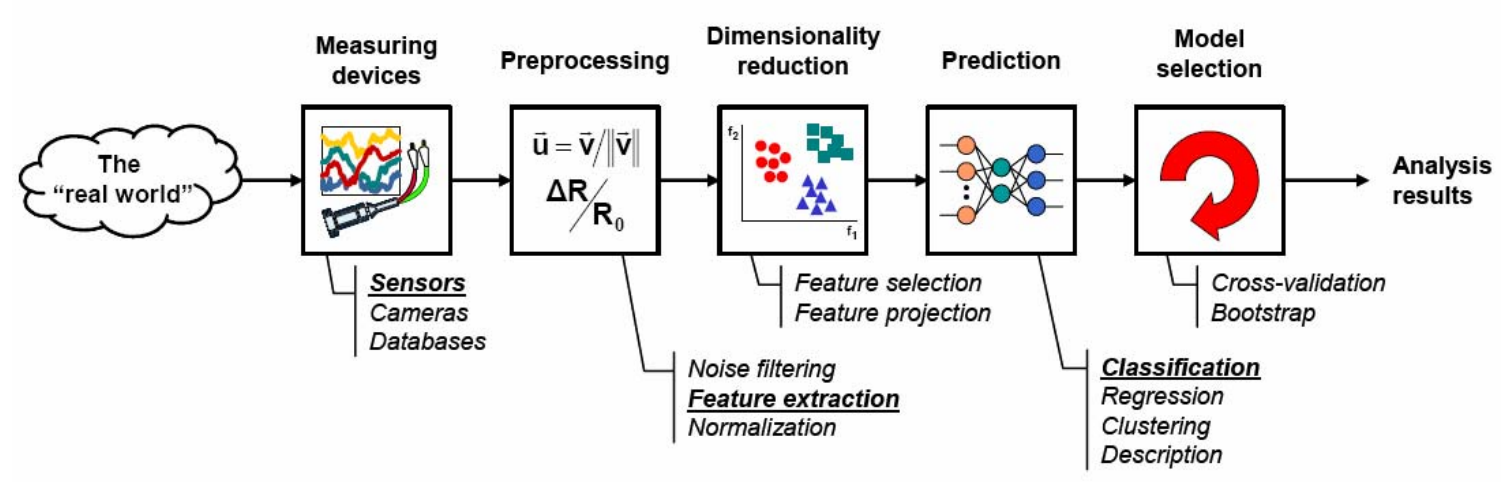

Fig 28. Stages of a pattern recognition problem (Gutierrez-Osuna, 1998). 
This chapter is not intended as a complete reference for techniques that can be utilized at each stage. Instead, our discussion simply focuses on those techniques that will be employed on the experimental analyses performed in the following chapter. Most of the algorithms mentioned in this chapter, and many other mathematical and statistical techniques for analytical chemistry, can be found under the denomination of chemometrics. Nevertheless, we will retain the term pattern recognition when referring to such techniques.

Successful detection and discrimination of analytes rely heavily upon the choice of signal processing technique employed. Therefore, bearing an understanding about signal processing approaches is critical for the correct interpretation of the data generated by IRIS. We organize the discussion of calibration and pattern classification as follows. In sections IV.1 through IV.3, we discuss techniques for spectral acquisition, preprocessing, and feature selection which are important for both calibration and classification problems. Subsequently, in section IV.4, we discuss calibration methods which are used to build predictive mathematical models for concentration on an analyte in a matrix of absorbing species. Section IV.5 provides classifiers for pattern recognition. Finally, in section IV.6, we present a validation strategy that is employed in calibration and classification techniques used throughout the experimental chapter of this manuscript. 


\section{IV.1. Acquisition of spectra}

\section{IV.1.1. Chemical modulation with lock-in amplification}

Lock-in amplifiers are commonly used to extract signals with a known carrier from a noisy environment. The signal to be measured is modulated with a carrier frequency, then amplified with a lowpass filter tuned to the carrier frequency (EG\&G Princeton Applied Research, 1984).

In addition to the LVF and 64-pixel pyroelectric detector, the $\mu$ Array device integrated in IRIS includes a built-in lock-in amplification circuit (IR Microsystems, 2006). This circuit uses the frequency from the modulated light (obtained by chopping or pulsing), which is necessary for the normal operation of the pyroelectric sensor array, as a carrier frequency to also to cancel the effect of high frequency noise captured by the detector array. Specifically, this lock-in amplifier circuit uses the $10 \mathrm{~Hz}$ frequency of the chopper as a carrier frequency. The amplified signal is averaged over a period of 1 second and transmitted via the RS232 cable to the computer.

The idea of lock-in amplification can be further extended as a sampling technique by intermittently delivering the chemical vapors to the gas cell. In this case, the carrier frequency is determined by the frequency of chemical modulation. Signal acquisition needs to be performed continuously, and the chemical modulation frequency has to be lower than the sampling or noise frequency, so that the latter can be averaged out. Throughout this manuscript, we will refer to this technique as chemical modulation with lock-in amplification (CM/LA). 


\section{IV.1.2. Signal integration}

In the signal-integration mode of operation, the reference spectrum is acquired and averaged for a certain period of time, followed by acquisition and averaging of sample spectra for a same length period. The rationale behind this method is that averaging of each spectra over a (relatively long) period of time eliminates uncorrelated noise (See section III.3.1.1). The resulting signals are used to calculate the infrared absorption of the measurand according to Beer-Lambert's Law.

\section{IV.1.3. CM/LA vs. integration time}

As previously discussed in section III.3.1.1, precision errors are typically interpreted as zero-mean random variables whose uncertainties can be reduced by replication. Here we discuss the merits of the two methods that utilize replication as a way to decrease precision error: $\mathrm{CM} / \mathrm{LA}$ and signal integration.

In IRIS, the use of $\mathrm{CM} / \mathrm{LA}$ as a sampling technique can be particularly beneficial during the device's initialization period (See section III.3.5). During initialization, sensor drift prevents long interval periods between reference and sample measurements, thus typically making $\mathrm{CM} / \mathrm{LA}$ a more attractive technique to achieve signal averaging than integration. However, when the system reaches stability, there is a tradeoff between $\mathrm{CM} / \mathrm{LA}$ and integration. For the same number of measurements, $\mathrm{CM} / \mathrm{LA}$ takes longer because of the additional time needed to clean and pressurize the system, which are required twice with every modulation cycle. However, since the averaging of repeated measurements leads to cancellation of random noise, $\mathrm{CM} / \mathrm{LA}$ also results in a signal that is less affected by imprecise pressurization. 


\section{IV.1.4. Difference spectra}

Difference spectra is a technique commonly used to uncover embedded infrared absorption spectra from a matrix or mixture. This is carried out by simply subtracting the infrared spectrum of one or more components from the combined system (Stuart, 2004). For instance, the infrared absorption spectrum of a sample contained in a mixture can be revealed by subtracting the infrared absorption spectrum of the matrix from the mixture. It is important to notice that if mixture components interact, the difference spectrum may reflect this behavior as positive or negative peaks.

In IRIS, difference spectra is particularly useful if the matrix has strong absorption peaks in LVF region, which makes it difficult to interpret visually the sample's infrared absorption spectrum. For instance, water and water vapor are common matrix components that show significant infrared absorption in the LVF region, and can be subtracted from the mixture by way of difference spectra.

\section{IV.1.5. Deconvolution}

Deconvolution aims to remove distortions caused by the instrument on the underlying signal. The process of deconvolution for an infrared absorption spectroscope consists of compensating for the intrinsic linewidths of bands, making it possible to resolve overlapping bands. In IRIS, moving the infrared emitter closer to the detector leads to worse collimation and increased full width at half maximum (FWHM) of wavelength coverage at every pixel (See section III.3.4). Therefore, in a situation where the source and detector must be close (i.e. less than $10 \mathrm{~cm}$ ), such as for the analysis of liquid samples, deconvolution may reveal spectral features which are otherwise blurred away. 


\section{IV.2. Preprocessing}

Preprocessing the data is a critical step for spectral analysis and classification. Preprocessing can be employed as a preliminary step for exploratory data analysis, or as a tool for transforming the data prior to calibration/classification. In this section, we will outline and discuss the preprocessing techniques that are used for the analysis of experimental data obtained with IRIS.

\section{IV.2.1. Normalization}

Normalization is the last preprocessing step prior to classification (Gutierrez-Osuna et al., 2003). The goal of normalization is to adjust the measurand (i.e., range, offset, variance) in order to obtain the most meaningful sample inter-comparisons. Three normalization procedures are briefly discussed in this section: autoscaling, sensor normalization, and vector normalization. It is important to understand which normalization procedure to use, since using the wrong technique may remove or mask critical information in the spectra. Furthermore, there are instances when more then one technique is appropriate (Egan et al., 2003).

Autoscaling performs centering and normalization to unit variance on a pixel-by-pixel basis. Centering is performed by calculating a set-mean spectrum $\bar{x}_{k}$ for each wavenumber, and subtracting it from each sample:

$$
x_{i, k}^{\text {centered }}=x_{i, k}-\bar{x}_{k}
$$


where $x_{i, k}$ represents the recorded absorption value from the $\mathrm{i}^{\text {th }}$ sample at the $\mathrm{k}^{\text {th }}$ wavelength. Autoscaling is then completed by dividing the centered spectrum by the standard deviation $s_{k}$ calculated at each wavelength:

$$
x_{i, k}^{\text {autoscaled }}=\frac{x_{i, k}^{\text {centered }}}{s_{k}}
$$

Autoscaling is typically used when we want to promote that each wavelength in the spectrum has the same influence upon the classifier, regardless of its dynamic range. On the downside, autoscaling can introduce noise from wavenumbers with weak absorption.

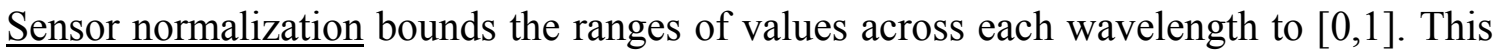
is achieved by subtracting the minimum and dividing by the range of the wavelength across the entire dataset (Gutierrez-Osuna et al., 2003):

$$
x_{i, k}^{\text {normalized }}=\frac{x_{i, k}-\min _{\forall i}\left[x_{i, k}\right]}{\max _{\forall i}\left[x_{i, k}\right]-\min _{\forall i}\left[x_{i, k}\right]}
$$

where $x_{i, k}$ refers to the absorption value of the $\mathrm{i}^{\text {th }}$ sample at the $\mathrm{k}^{\text {th }}$ wavelength. Similarly to autoscaling, sensor normalization ensures that the magnitude of each wavelength is comparable, thus preventing large absorptions at a single wavelength from overwhelming the classifier. Likewise, it can also introduce noise from weak absorption lines. Unlike autoscaling, however, sensor normalization is sensitive to outliers. 
In vector normalization, each spectrum is scaled such that it becomes a unit vector (i.e. a vector whose length is 1 ). This is achieved by dividing the value at each wavelength $\mathrm{k}$ in a spectrum $i$ by the norm of the spectrum:

$$
\hat{x}_{i, k}=\frac{x_{i, k}}{\left\|x_{i}\right\|}=\frac{x_{i, k}}{\sqrt{\sum_{k \in i} x_{i, k}^{2}}}
$$

Imprecision in the instrumentation may lead to experimental variations of concentration in the sample cell for repeated measurements. Since concentration effects are linear with respect to infrared absorption measurements (See section II.4.1.1), these unintentional variations of concentrations results in a proportional variation in the infrared absorption measurements. A treatment of the data using vector normalization removes these concentration effects, and is particularly useful in pattern classification, where concentration information is irrelevant. However, vector normalization should not be employed in applications where concentration effects carry important information (i.e., calibration).

\section{IV.3. Feature selection and extraction}

Each wavenumber in a spectrum can be treated as an independent variable that conveys unique information about the chemical structure of an analyte or the composition of a mixture. However, a typical FTIR spectroscope, which can record spectra at resolutions of $0.125 \mathrm{~cm}^{-1}$, produces tens of thousands of unique infrared absorption measurements at different wavenumbers in the mid infrared region $\left(4000-600 \mathrm{~cm}^{-1}\right)$. In order to make the classification of spectral patterns computationally efficient, dimensionality reduction 
techniques should be employed in these cases (See sections IV.3.1 and IV.3.2). In addition, dimensionality reduction techniques are useful in exploratory data analysis because they assist with the visualization of high-dimensional data, i.e., by transformation into a lower-dimensional space (Brereton, 2002).

IRIS generates infrared absorption information on 64 wavenumbers, which implies 64 dimensions. Therefore, IRIS suffers much less from dimensionality problems than high-resolution infrared spectrometers. Nonetheless, these techniques are still extremely useful to highlight differences or provide visual cues as to what are the main relationships between spectra and analytes.

\section{IV.3.1. Feature extraction}

In this section we discuss two popular feature extraction techniques: Principal Component Analysis (PCA) and Linear Discriminant Analysis (LDA). PCA is a dimensionality reduction technique that attempts to retain as much of the variance in the original high-dimensional space as possible (Loeve, 1963). Fundamentally, this is achieved by projecting the original data onto the eigenvectors corresponding to the largest eigenvalues of the covariance matrix. As a result, the coordinate axes are transformed and aligned with the directions of highest variance. The eigenvectors and the projections of the data onto them are commonly referred to as the loadings and the scores, respectively. A detailed algorithmic discussion of PCA for spectral analysis can be found in (Fukunaga, 1990).

In contrast to PCA, LDA takes class labels into account. LDA is a dimensionality reduction technique that attempts to retain as much class discriminatory information as 
possible (Fisher, 1936). This is achieved by finding a projection that maximizes the ratio of between-class $S_{\mathrm{B}}$ to within-class $S_{\mathrm{W}}$ scatter. Specifically, LDA tries to maximize the objective function:

$$
J(W)=\frac{\left|W^{T} S_{B} W\right|}{\left|W^{T} S_{W} W\right|}
$$

where $\mathrm{W}$ is the projection matrix, and the scatter matrices $S_{\mathrm{W}}$ in defined as follows:

$$
S_{W}=\sum_{i=1}^{C} \sum_{x \in \omega_{i}}\left(x-\mu_{i}\right)\left(x-\mu_{i}\right)^{T}
$$

where $\mu_{i}=\frac{1}{N} \sum_{x \in \omega_{i}} x$ is the mean of the $\mathrm{i}$-th class, $\mathrm{C}$ is the total number of classes, $\mathrm{N}$ is the total number of observations, and $\omega_{\mathrm{i}}$ refers to the $\mathrm{i}^{\text {th }}$ class. In turn, $S_{\mathrm{B}}$ is defined as follows:

$$
S_{B}=\sum_{i=1}^{C} N_{i}\left(\mu_{i}-\mu\right)\left(\mu_{i}-\mu\right)^{T}
$$

where $\mu=\frac{1}{N} \sum_{\forall x} x=\frac{1}{N} \sum_{x \in \omega_{i}} N_{i} \mu_{i}$ is the pooled mean, and $\mathrm{N}$ is the total number of observations.

Thus, the directions that maximize the objective function are those that maximize between-class separability while minimizing within-class variability. Furthermore, since the rank $S_{B}$ is at most C-1 (i.e., because of the constraint $\mu=\frac{1}{N} \sum_{x \in \omega_{i}} N_{i} \mu_{i}$ ), the data can be projected onto a space with at most $\mathrm{C}-1$ dimensions. 


\section{IV.3.2. Feature selection}

The goal of feature selection is to find the best subset of features that explains the data. Unlike feature extraction, this dimensionality reduction technique does not perform a transformation of the coordinate space, and is particularly useful if further manipulation requires that features retain interpretable measurement units.

Albeit the best subset could be obtained by investigating every possible combination features, this is typically computationally unfeasible for a large feature space. In such case, suboptimal strategies which favor computational efficiency should be employed. The selection criteria of features in pattern-recognition problem generally focus on the minimization of classification error rate or the maximization of inter-class distances. In this section we discuss two popular feature selection methods: sequential forward selection, and sequential backward selection.

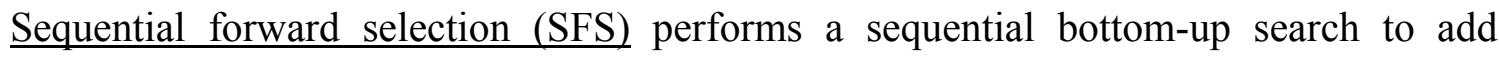
features to a feature set, one at a time, until a final feature set is reached. Specifically, SFS initializes a feature set $X$ to null, and proceeds by iteratively adding features that maximizes the following criterion function (Webb, 2002):

$$
J_{j}=J\left(X+\xi_{j}\right)
$$

where $\xi_{j}$ refers to the $\mathrm{j}^{\text {th }}$ feature (i.e. the $\mathrm{j}^{\text {th }}$ pixel of the detector array in IRIS). As mentioned above, the specific function $J()$ is typically based on classification error rate or class separability (Webb, 2002). The main drawback of SFS is that once features are 
added, they cannot be removed from the subset. This becomes a problem if previously added features become redundant due to newly added features.

Sequential backward selection (SBS) performs a top-down elimination of features from the feature set until a final feature set is reached. SBS estimates how each feature contained in the feature set affects the system, and eliminates the feature that contributes the least. Specifically, SBS initializes the feature set to contain all features, and proceeds by iteratively removing the features that maximizes the following criterion function (Webb, 2002):

$$
J_{j}=J\left(X-\xi_{j}\right)
$$

where $\xi_{j}$ refers to the $\mathrm{j}^{\text {th }}$ feature (i.e. the $\mathrm{j}^{\text {th }}$ pixel of the detector array in IRIS). Similarly to SFS, the execution steps can not be retracted, and therefore once a feature is eliminated, it can not be reinserted.

Floating versions of these algorithms have been shown to provide near-optimum results (Pudil et al., 1994). Such methods are freely allowed to correct wrong decisions in regards to adding or removing features made in previous steps. Thus, this backtracking process enables the algorithm to find a near-optimal solution.

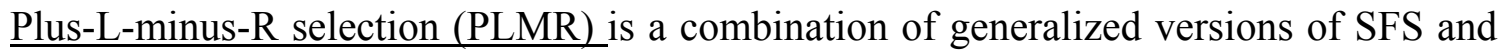
SBS. In a forward search (i.e. $\mathrm{L}>\mathrm{R}$ ), the algorithm consists of first adding $\mathrm{L}$ and then removing $\mathrm{R}$ features that maximizes some criteria function. The algorithm initializes the feature subset to null, and proceed by iteratively performing SFS for L times, followed 
by SBS for $\mathrm{R}$ times. In a backward search (i.e. $\mathrm{L}<\mathrm{R}$ ), the algorithm proceeds in the reverse order. Unlike SFS and SBS, the algorithm can retract steps and avoid redundancy of features to a degree of selectivity specified in the L and R parameters.

\section{IV.4. Calibration}

Calibration is one of the most important tasks in quantitative infrared absorption analysis. It seeks to uncover the relationship between absorption and concentration in the presence of instrumental or experimental artifacts, and typically involves regression analysis for the development of a predictive mathematical model. Beer-Lambert's Law (See section II.4.1.1) predicts a linear relationship between absorption and concentration, which has been proven empirically for low absorption values (Adams, 2004). In a simple linear model, the estimated absorption $\hat{y}_{i}$ of a given analyte depends on its concentration $x_{i}$ as follows:

$$
\hat{y}_{i}=a+b x_{i}
$$

where $a$ and $b$ are constant coefficients that represent the bias and the gradient of the regression line, respectively. This model can be further simplified by assuming that the matrix containing the analyte is the reference, which leads to the elimination of the bias coefficient and a regression through the origin:

$$
\hat{y}_{i}=b x_{i}
$$

The relationship between absorption $\hat{y}_{i}$ and concentration $x_{i}$ is given by the coefficient $b$, which can be used to estimate unknown concentration from recorded spectra: 


$$
\hat{x}_{i}=\frac{y_{i}}{b}
$$

The assumption of a linear model is not always correct (See section III.3.1.2). Discussions about more complex non-linear models, such as polynomial regression, as well as details about errors and goodness of fit can be found elsewhere (Adams, 2004; Mark \& Workman Jr., 2003).

Up to this point, the discussion has focused on estimating concentration $\hat{x}_{i}$ from a single independent variable $y_{i}$. In reality, the spectrum obtained by IRIS contains simultaneous measurements at 64 different wavelengths, which requires the use of a more sophisticated approach, termed multivariate regression. In multivariate regression, not all wavelengths in the spectrum contribute in an equal manner to the construction of a good prediction mathematical model. In particular, when calibrating for analytes in the presence of other absorbing species, using certain wavelengths may in fact hinder the performance of the final model. Furthermore, with high resolution spectroscopes, the use of the entire spectrum for calibration becomes computationally intensive. Therefore, performing a selection of variables for regression is often important. The treatment of variable (a.k.a. feature) selection is deferred to section IV.3.

Commonly used statistical methods for multivariate regression include classical least squares (CLS) (Adams, 2004), inverse least squares (ILS) (Adams, 2004), principal component regression (PCR) (Vigneau et al., 1997), partial least squares regression (PLSR) (Geladi \& Kowalski, 1986) and soft independent modeling of class analogy (SIMCA) (Wold, 1976) . A brief presentation of each method follows. 


\section{IV.4.1. Classical least squares (CLS)}

In CLS (also known as the K-Matrix method), a regression model is built based on the recorded mixture matrix and its corresponding composition matrix, as follows:

$$
Y=K \times X
$$

where each column in $Y(\mathrm{n} \times \mathrm{m})$ represents the measured spectrum for a mixture, each column in $X(\mathrm{c} \times \mathrm{m})$ represents the concentration of each mixture, $K(\mathrm{n} \times \mathrm{c})$ is the sensitivity matrix we want to obtain, $n$ represents the number of wavelengths (i.e. 64 in IRIS), $m$ represents the number of mixtures, $c$ represents number of analytes in a mixture. $K$ is obtained via inverse squares as follows:

$$
K=Y \times X^{T} \times\left(X \times X^{T}\right)^{-1}
$$

The estimated concentration $\hat{X}$ of an unknown mixture $Y u$ can then be calculated as follows:

$$
\hat{X}=\left(K^{T} \times K\right)^{-1} \times K^{T} \times Y u
$$

CLS is a intuitive extension of ordinary least squares applied to multivariate regression, but in order to be used it requires knowledge of the complete composition of the calibration mixture (i.e. the concentration all absorbing species). When there is only partial information about the composition of the mixture, an alternative method such as ILS must be applied. 


\section{IV.4.2. Inverse least squares (ILS)}

In the ILS regression model (also known as the P-Matrix method), the dependent variable is the concentration matrix and the independent variable is the mixture matrix as follows:

$$
X=P \times Y
$$

where columns in $Y(\mathrm{n} \times \mathrm{m})$ represents the measured spectrum for a mixture, each column in $X(\mathrm{c} \times \mathrm{m})$ represents the concentration of each mixture, $P(\mathrm{c} \times \mathrm{n})$ is the calibration matrix we want to obtain, $n$ represents the number of wavelengths (i.e. resolution) of the spectrum, $m$ represents the number of mixtures and $c$ is the number of known analyte concentrations in the mixture. $P$ is obtained via inverse squares as follows:

$$
P=X \times Y^{T} \times\left(Y \times Y^{T}\right)^{-1}
$$

The estimated concentration $\hat{X}$ of the mixture $Y u$ of unknown composition can then be estimated by using $P$ as follows:

$$
\hat{X}=P \times Y u
$$

In ILS, concentration is defined as a function of the recorded response spectra, and knowledge of the complete composition of the calibration mixture is not necessary. However, it requires that the number of calibration mixtures be larger than the number of features (i.e. wavelengths), and only the composition (i.e. concentration) information about analyte to be predicted is needed. To avoid overfitting, the number of samples generally has to be much larger then the number of variables. When there is a limitation to the number of samples, a feature selection technique (See section IV.3) is typically 
employed to select only the features that are relevant to the regression analysis. Furthermore, collinearity of features may add redundancy and hinder the performance of the resulting ILS prediction model. If collinearity of variables poses a problem, an alternative regression analysis method such as PCR should be used.

\section{IV.4.3. Principal component regression (PCR)}

Fundamentally, PCR combines PCA (See section IV.3.1) with a multivariate regression analysis technique such as ILS. PCR uses PCA to obtain the principal components of the mixture matrix, and performs ILS on the transformed coordinate space. Consequently, since the principal components are orthogonal and in the direction of highest variation, PCR avoids feature collinearity issues that might be present if only ILS was used. Specifically, the PCR heuristic is as follows:

Step 1. Perform mean-centering for the concentration vector $x$ and spectral matrix $Y$.

$$
\begin{aligned}
& x^{\text {centred }}=x-\bar{x} \\
& Y^{\text {centred }}=Y-\bar{Y}
\end{aligned}
$$

Step 2. Perform PCA on $Y^{\text {centred }}$, and retrieve the loadings (eigenvectors) and scores (principal components) for the desired number of principal components $q$.

$$
\begin{gathered}
\text { loadings, scores } \leftarrow P C A\left(Y^{\text {centered }}\right) \\
S=\text { scores }_{1 . . q} \\
L=\text { loading }_{1 . . q}
\end{gathered}
$$




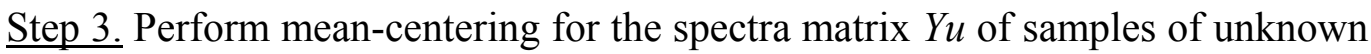
composition.

$$
Y u^{\text {centred }}=Y u-\bar{Y}
$$

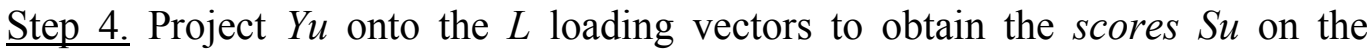
principal components subspace.

$$
S u=Y u^{\text {centred }} \times L
$$

Step 5. Perform ILS and adjust the bias to obtain the estimates for concentration.

$$
\begin{gathered}
\hat{x}=\operatorname{ILS}\left(x^{\text {centred }}, S, S u\right) \\
\hat{x}=\hat{x}+\bar{x}
\end{gathered}
$$

where the column vector $x(\mathrm{~m} \times 1)$ contains the concentration of the analyte for $\mathrm{m}$ examples; rows of matrix $Y(\mathrm{~m} \times \mathrm{n})$ are the corresponding spectra; rows of matrix $Y u(\mathrm{r} \times$ n) are the spectra of $r$ examples of unknown composition; $\hat{x}(r \times 1)$ is the estimated concentration vector corresponding to $Y u ; L(\mathrm{n} \times \mathrm{q})$ and $S(\mathrm{~m} \times \mathrm{q})$ contain the ordered (i.e. by decreasing variability) $q$ principal components coefficients and projections, respectively; $\mathrm{n}$ represents the number of wavelengths of the spectrum (i.e. resolution, which in IRIS corresponds to the number of pixels: $n=64), m$ represents the number of mixtures and $c$ is the number of known analyte concentrations in the mixture.

PCR generally works well when variability in the mixture matrix $Y$ can be strongly attributed to the calibration analyte. If the calibration analyte does not provide a major source of variability in the multi-component mixture, an alternative regression model, such as PLSR, may be more appropriate. 


\section{IV.4.4. Partial least squares regression (PLSR)}

Analogous to PCR, PLSR combines some aspects of principal component analysis with multivariate regression analysis. PLSR is particularly useful when trying to predict a small set of concentrations from high-resolution spectra (Abdi, 2003). PLSR differs from PCR by attempting to minimize the potential effects of wavelengths having large variances but which are irrelevant to the calibration model. This is achieved by finding a set of components, referred to as latent vectors, which decompose the concentration matrix $X$ and the spectral matrix $Y$, with the constraint that they explain as much about the covariance between $X$ and $Y$ as possible. In order to achieve this, a stepwise algorithm calculates the loading and score matrices one vector at a time in the order of their contribution to the variance until the desired model has been obtained. There are many implementations for PLSR, which typically target either prediction or calibration problems. Since we are concerned with calibration, we present a PLSR algorithm, named PLS1, for calibration (Haaland \& Thomas, 1988). The PLS1 algorithm is as follows

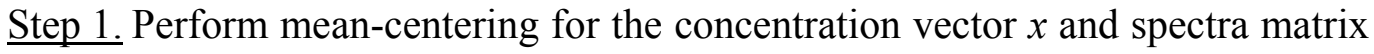
$Y$. Centering eliminates the need to fit a nonzero intercept and can reduce the number of factors required to construct a model.

$$
\begin{aligned}
& x=x-\bar{x} \\
& Y=Y-\bar{Y}
\end{aligned}
$$

Step 2. Form a weight vector $\hat{w}_{h}$ by obtaining a least squares solution to $Y=x \times w_{h}^{T}+E_{Y}$. 


$$
\hat{w}_{h}=Y^{T} \times x \times\left(x^{T} x\right)^{-1}
$$

$$
\text { normalize } \hat{w}_{h}
$$

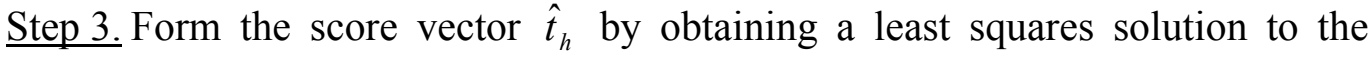
model $Y=t_{h} \times \hat{w}_{h}^{T}+E_{Y}$.

$$
\hat{t}_{h}=Y \times \hat{w}_{h} \times\left(\hat{w}_{h}^{T} \times \hat{w}_{h}\right)^{-1}=Y \times \hat{w}_{h}
$$

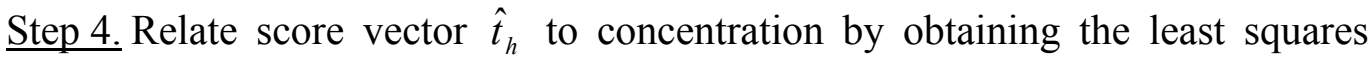
solution to $x=v_{h} \times \hat{t}_{h}+e_{x}$.

$$
\hat{v}_{h}=\hat{t}_{h} \times x \times\left(\hat{t}_{h}^{T} \hat{t}_{h}\right)
$$

Step 5. Form the PLS loading vector for $Y, \hat{b}_{h}$, by obtaining the least squares solution to the model $Y=\hat{t}_{h} \times b_{h}^{T}+E_{Y}$.

$$
\hat{b}_{h}=Y \times \hat{t}_{h} \times\left(\hat{t}_{h}^{T} \hat{t}_{h}\right)
$$

Step 6. Calculate the spectral $E_{Y}$ and concentration e residuals for $\mathrm{Y}$ and $\mathrm{x}$, respectively.

$$
\begin{gathered}
E_{Y}=Y-\hat{t}_{h} \times \hat{b}_{h}^{T} \\
e_{x}=x-\hat{v}_{h} \times \hat{t}_{h}
\end{gathered}
$$

Step 7. Increment h, substitute $E_{Y}$ for $Y$ and $e_{x}$ for $x$ in Step 2 and continue for desired number of loading vectors 
The concentration $\hat{x}$ of an analyte embedded in a mixture of unknown composition can then be estimated by using $B$ and $b_{0}$ as follows:

$$
\begin{aligned}
& b_{0}=\bar{x}-\bar{Y} \times \hat{b}_{h} \\
& \hat{x}=b_{0}+\hat{b}_{h} \times Y u
\end{aligned}
$$

The selection of the optimum number of factors is important for the performance of the prediction model. As with the number of principal components in PCR, the use of too many latent vectors may lead to overfitting. Thus, an optimum number of factors consists of typically the least number of factors that does not significantly increase prediction error variance. An optimal number of factors can be selected via crossvalidation, in a manner described in Section IV.6.1.

\section{IV.5. Classification}

As mentioned previously, detection, identification and classification of analytes in a blank or a mixture can be solved with pattern-recognition techniques. Classification is an integral part of any pattern-recognition problem. Classification consists of assigning a physical object or event to one of several pre-specified categories (Duda et al., 2001). A myriad classification techniques have been developed in the statistical and neural machine learning domains. Typically, the optimal choice for a classifier is problemdependent. In this section, we will discuss classification methods employed in the experimental evaluation of IRIS: the quadratic classifier, which belongs to the family of parametric techniques, and the nearest neighbor classifier, which is a representative from non-parametric techniques. 


\section{IV.5.1. Quadratic classifiers}

A quadratic classifier assumes that each class-conditional density is normally distributed (i.e., in a 64-dimensional space for IRIS). A minimum-error-rate classification can be achieved by classifying the analyte $x$ to the class $\omega$ the maximizes the following discriminant function (Duda et al., 2001):

$$
g_{i}(x)=\ln p\left(x \mid \omega_{i}\right)+\ln P\left(\omega_{i}\right)
$$

where

$$
\ln p\left(x \mid \omega_{i}\right)=-\frac{1}{2}\left(x-\mu_{i}\right)^{t} \sum_{i}^{-1}\left(x-\mu_{i}\right)-\frac{d}{2} \ln 2 \pi-\frac{1}{2} \ln \left|\sum_{i}\right|
$$

Which, after dropping the constant term $-\frac{d}{2} \ln 2 \pi$, can be expressed as

$$
g_{i}(x)=x^{t} W_{i} x+w_{i}^{t} x+w_{i 0}
$$

with

$$
\begin{gathered}
w_{i}=\sum_{i}^{-1} \mu_{i}, \\
W_{i}=-\frac{1}{2} \sum_{i}^{-1}, \text { and } \\
w_{i 0}=-\frac{1}{2} \mu_{i}^{t} \sum_{i}^{-1} \mu_{i}-\frac{1}{2} \ln \left|\sum_{i}\right|+\ln P\left(\omega_{i}\right)
\end{gathered}
$$

where $x$ is the 64-component column vector that represents the infrared absorption spectrum; $\mu_{i}$ is the 64-component mean vector of the $i^{\text {th }}$ class; $\sum_{i}$ is the covariance matrix of the $\mathrm{i}^{\text {th }}$ class; and $P\left(\omega_{i}\right)$ is the prior probability of the $\mathrm{i}^{\text {th }}$ class. 


\section{IV.5.2. K-nearest neighbors (KNN)}

In contrast to the quadratic classifier, the $\mathrm{KNN}$ algorithm does not assume an underlying probability distribution. KNN estimates the posteriori probability that class $\omega_{\mathrm{i}}$ is the correct class for sample $x$ by calculating the fraction of the $k$ nearest samples to $x$ that are labeled $\omega_{\mathrm{i}}$, as follows (Duda et al., 2001):

$$
P_{n}\left(\omega_{i} \mid x\right)=\frac{p_{n}\left(x, \omega_{i}\right)}{\sum_{j=1}^{c} p_{n}\left(x, \omega_{j}\right)}=\frac{k_{i}}{k}
$$

For all applications of KNN in this thesis, we assume the Euclidean distance to be the metric for the scalar distance between any two patterns.

\section{IV.6. Validation}

Validation is typically the last stage in a pattern classification or calibration problem. The validation stage seeks to determine the extent to which a trained model generalizes well for previously unseen data. It is particularly useful for model and parameter selection, as well as for estimating the true error rate of a classifier (Gutierrez-Osuna, 2002). In general, validation consists of splitting the data into training and validation sets. The training set is used to train the model, enabling it to adapt a set of metaparameters, while the validation set is used to measure the performance of the model. Among the most commonly used validation methods are holdout, bootstrap, k-fold and leave-one-out cross-validation. In this section we will discuss the leave-one-out crossvalidation method, which is the validation technique used throughout the experimental chapter of this manuscript. 


\section{IV.6.1. Leave-one-out cross-validation (LOO)}

As with other validation methods, LOO seeks to determine the extent to which a trained model generalizes well for previously unseen data. In LOO, an error rate is estimated by training the classifier with $n-1$ samples, and testing it with the remaining sample. This procedure is repeated $n$ times, until every sample in the dataset has been used as a testset (Webb, 2002). The final model is selected based on the average performance over all partitions. 


\section{CHAPTER V}

\section{EXPERIMENTAL}

Infrared absorption spectroscopy has proven to be a powerful analytical method for a vast number of applications, including quality control, industrial process monitoring, failure analysis, forensic identification and food fraud. The goal of this chapter is twofold. The first objective is to demonstrate the efficacy of IRIS as an analytical tool for gases. In this realm, we will show how IRIS can be used to estimate the concentration of an analyte in the presence of other infrared absorbing species in the gas phase. The second objective is to demonstrate the use of IRIS on real-world applications. In particular, we have selected two foodstuffs applications in the domain of edible oils and fats: oil adulteration and trans fatty acid detection. We chose this domain because oils and fats have well-resolved absorption bands in the mid infrared region, which can be assigned to chemical functional groups.

\section{V.1. Calibration}

In this section, we demonstrate the use of CLS, ILS, PCR and PLSR calibration techniques (See section IV.4) to build predictive mathematical models for the concentration of an analyte in a matrix of interfering absorbing species. In particular, we will demonstrate the calibration for acetone embedded in a matrix of water, isopropyl alcohol and witch hazel; these analytes were chosen so that we could obtain a mixture of simple (e.g. acetone) and complex (e.g witch hazel) absorbing species in highly concentrated gas form. We choose to focus on one analyte in the mixture in order to 
keep the discussion concise, but the model can be built for any of the mixture components. A comparison of the calibration results will also be provided.

\section{V.1.1. Experimental procedures}

Mixtures were prepared in liquid phase. No treatment of the analyte or mixture prior to sampling was performed. Each analyte was inserted with a $10 \mathrm{cc}$ syringe into a glass vial, whose headspace was directly placed in the gas flow path.

IR spectra were recorded from the headspace of the liquid mixtures, pressurized to 12 psig in the gas cell. Chemical modulation with lock-in amplification, with a carrier frequency of $0.05 \mathrm{~Hz}$, was used as a sampling technique to improve precision. Five cycles were executed per sample, and the resulting spectra were averaged.

\section{V.1.2. Spectra of the mixture components}

The mixtures consisted of four common household chemicals and solvents: isopropyl alcohol, acetone, witch hazel and water. Fig 29 through Fig 32 show the infrared absorption spectrum of acetone, isopropyl alcohol, water and witch hazel, respectively. For comparison purposes, the figures display the infrared spectra obtained by a highresolution FTIR instrument, as well as the spectra retrieved by IRIS. From these figures, we observe that the center wavelength of some strong absorption bands vary from one chemical to another, while others are very close, which enables the formulation of a quite interesting mixture problem. 


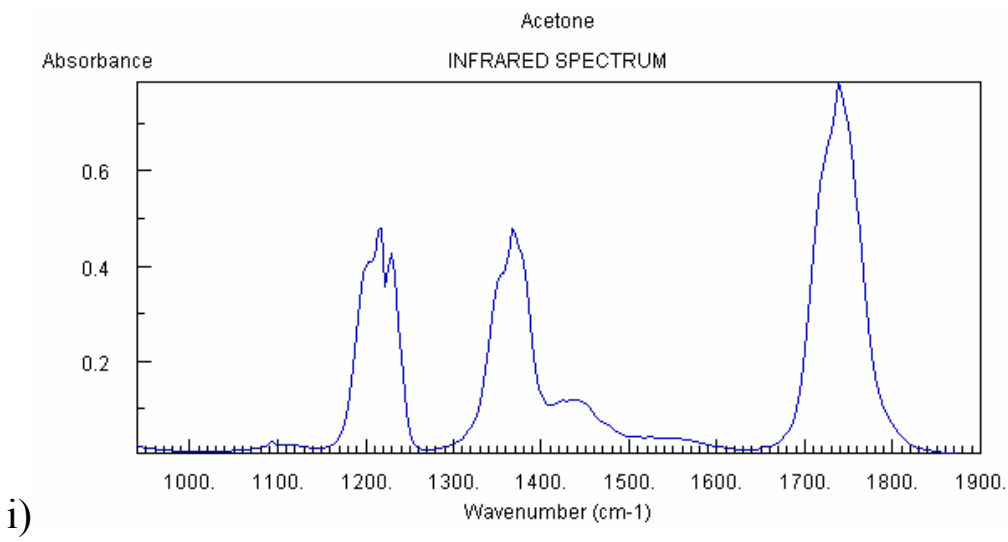

ii)

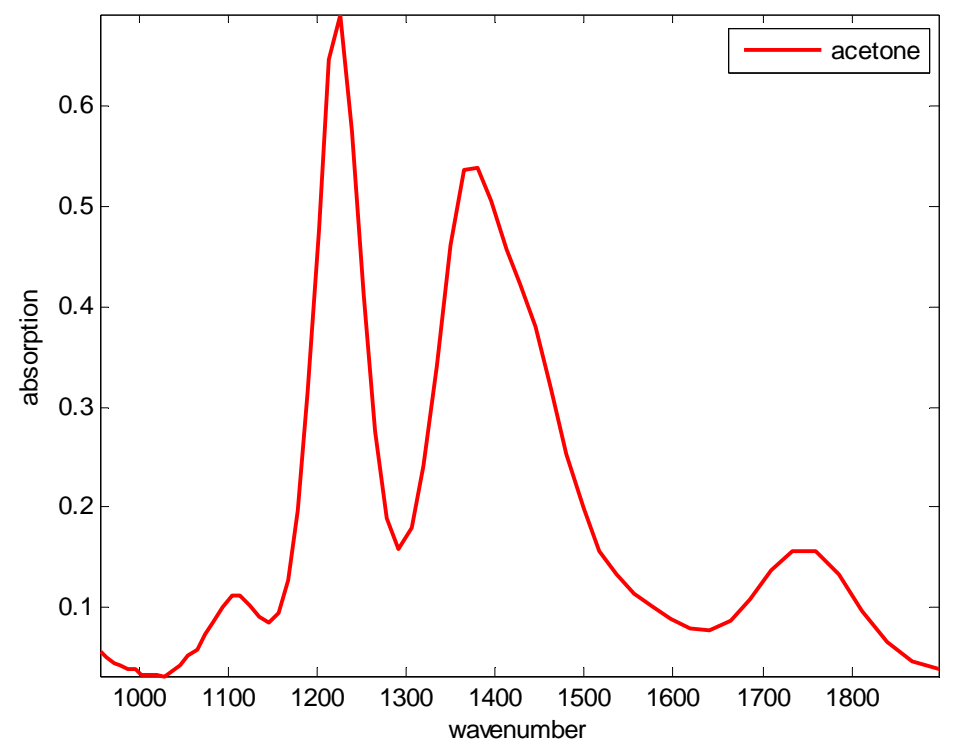

Fig 29. Infrared absorption spectrum of acetone in gas phase. i) High-resolution spectrum obtained via FTIR. Adapted from NIST (Linstrom \& Mallard, 2003). ii) Infrared absorption spectrum obtained by IRIS. 


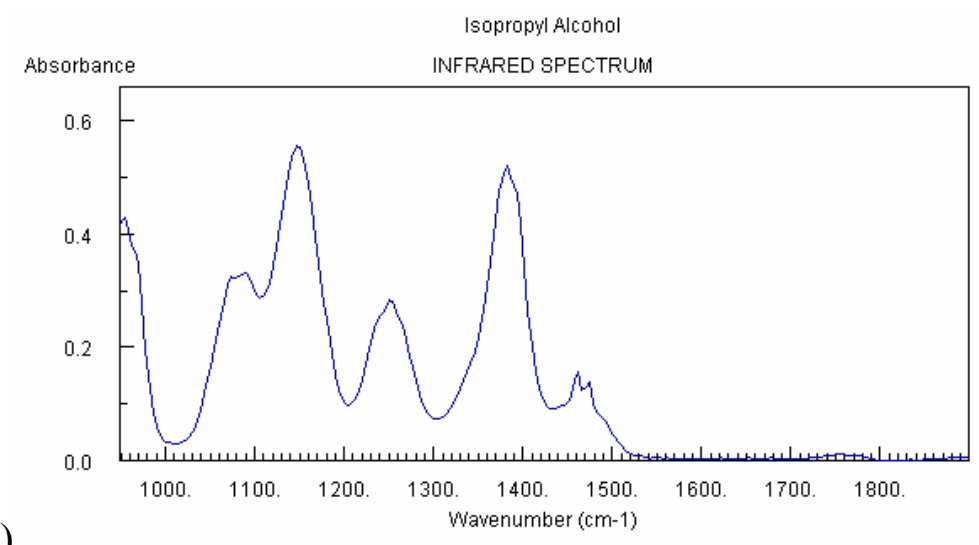

i)

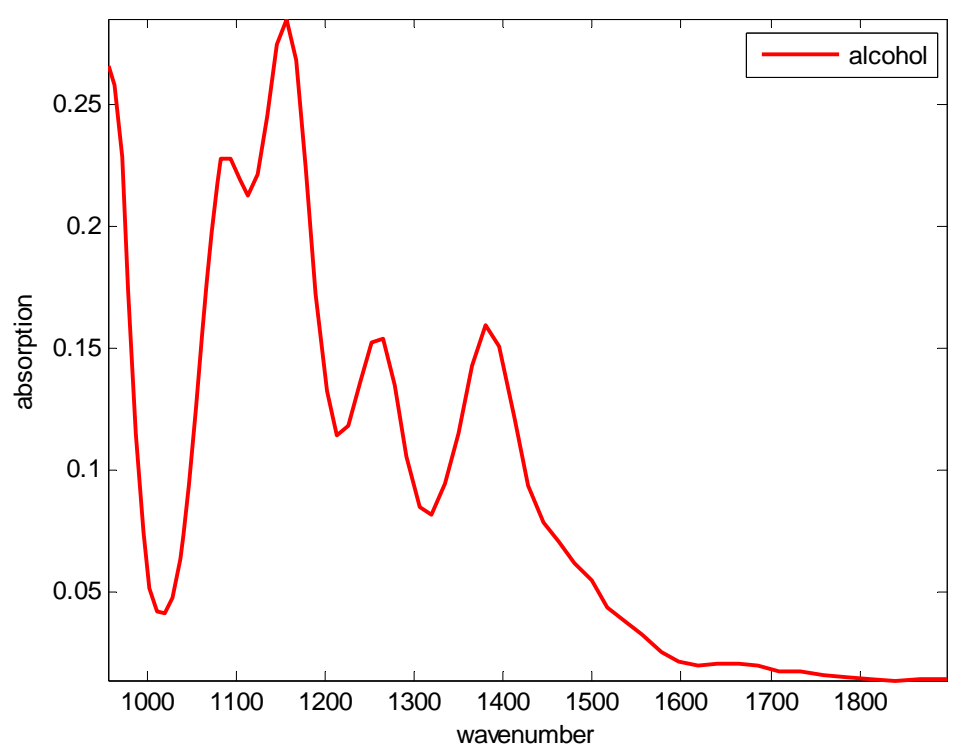

ii)

Fig 30. Infrared absorption spectrum of isopropyl alcohol in gas phase. i) Highresolution spectrum obtained via FTIR. Adapted from NIST (Linstrom \& Mallard, 2003). ii) Infrared absorption spectrum obtained by IRIS. 


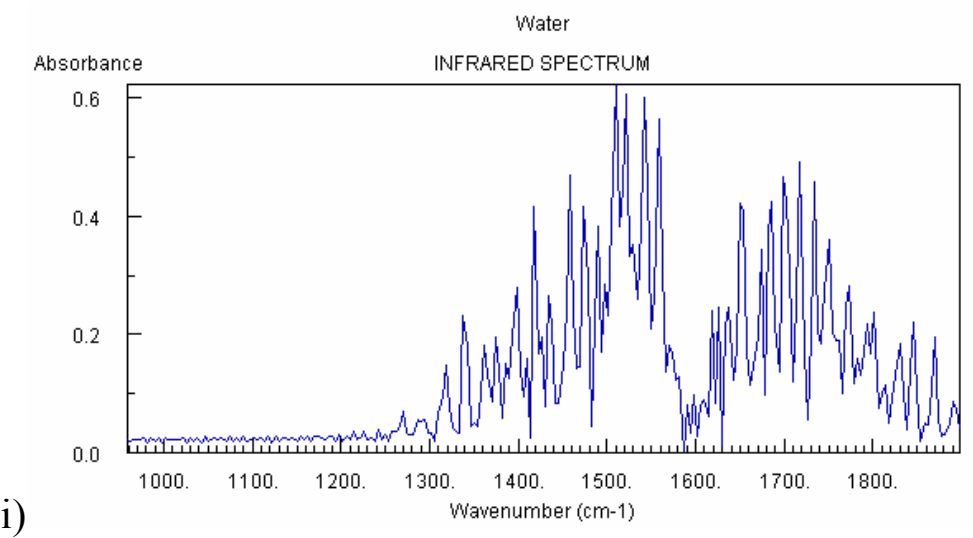

i)

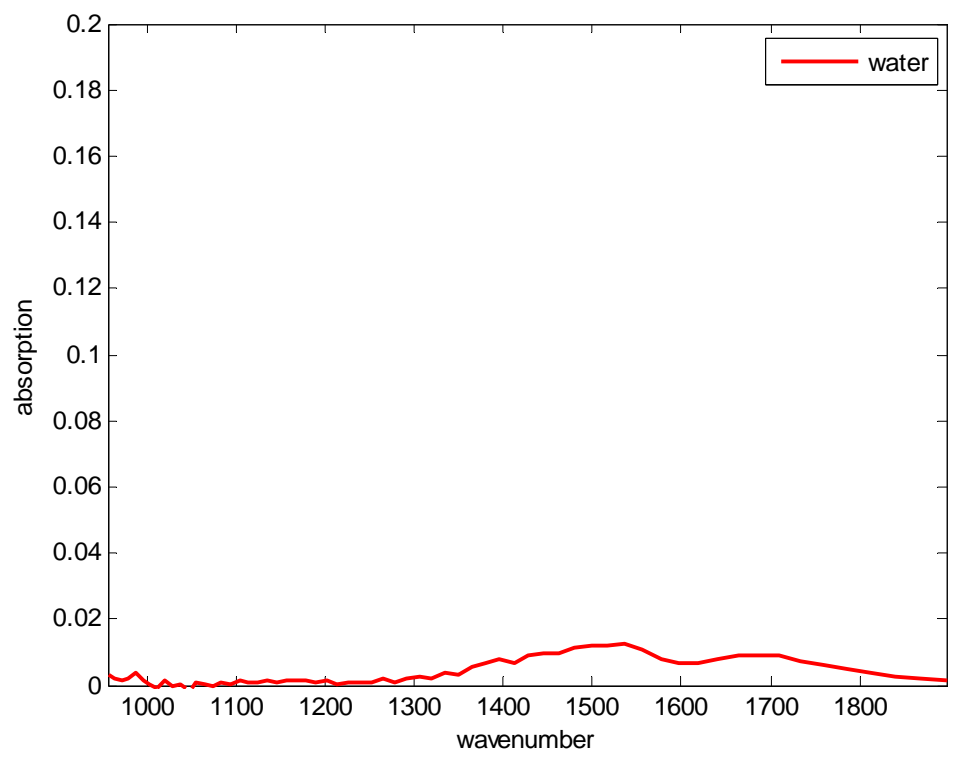

Fig 31. Infrared absorption spectrum of water in gas phase. i) High-resolution spectrum obtained via FTIR. Adapted from NIST (Linstrom \& Mallard, 2003). ii) Infrared absorption spectrum obtained by IRIS. 


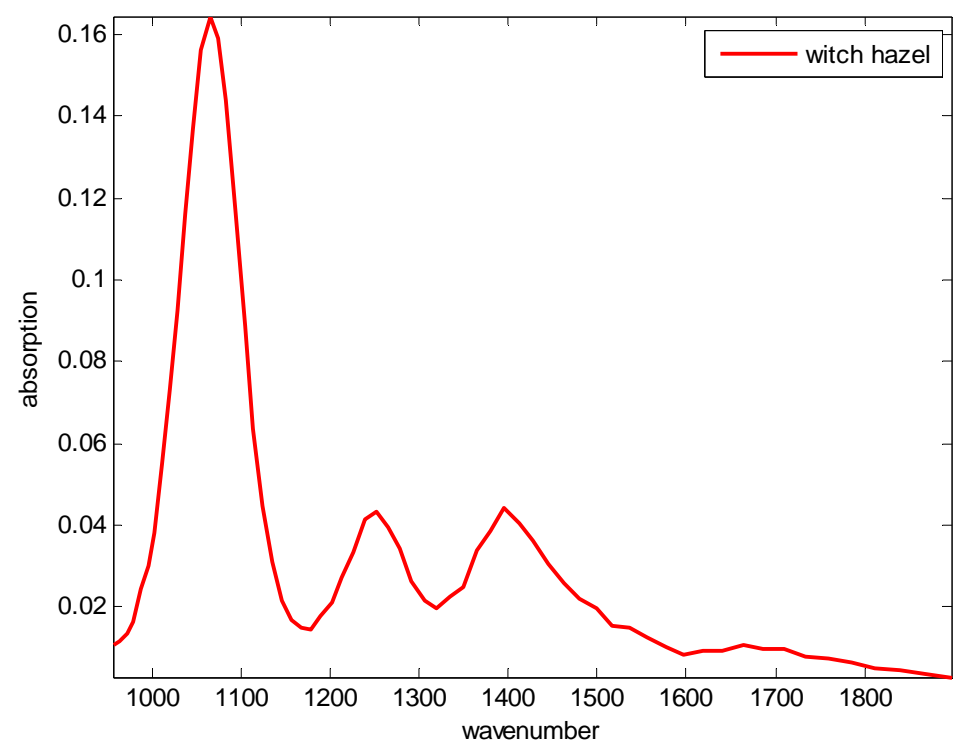

Fig 32. Infrared absorption spectrum of witch hazel in gas phase obtained by IRIS. FTIR spectra was not available at the NIST site.

All elements in the matrix showed important infrared absorption peaks centered at different wavenumbers in the LVF region. The application of the four calibration methods mentioned in section IV.4 follows.

\section{V.1.3. Results and discussions}

Thirteen mixtures with a range of acetone concentrations and random proportions of the matrix components were prepared, as summarized in Table 2. Fig 33 shows the corresponding infrared absorption spectra of the thirteen mixtures studied. 
Table 2 Mixture composition. The values in each column below the analytes indicate the percentage by weight of each component in the corresponding mixture.

\begin{tabular}{ccccc}
\hline No. & Acetone $(\%)$ & Witch hazel $(\%)$ & Water $(\%)$ & Alcohol $(\%)$ \\
\hline 1 & 6 & 5 & 90 & 0 \\
2 & 2 & 18 & 80 & 0 \\
3 & 4 & 0 & 50 & 46 \\
4 & 1 & 0 & 70 & 29 \\
5 & 8 & 0 & 80 & 12 \\
6 & 8 & 0 & 90 & 2 \\
7 & 2 & 0 & 80 & 18 \\
8 & 6 & 0 & 34 & 60 \\
9 & 10 & 30 & 60 & 0 \\
10 & 0 & 40 & 30 & 30 \\
11 & 0 & 40 & 30 & 30 \\
12 & 0 & 60 & 40 & 0 \\
13 & 10 & 10 & 70 & 10 \\
\hline
\end{tabular}

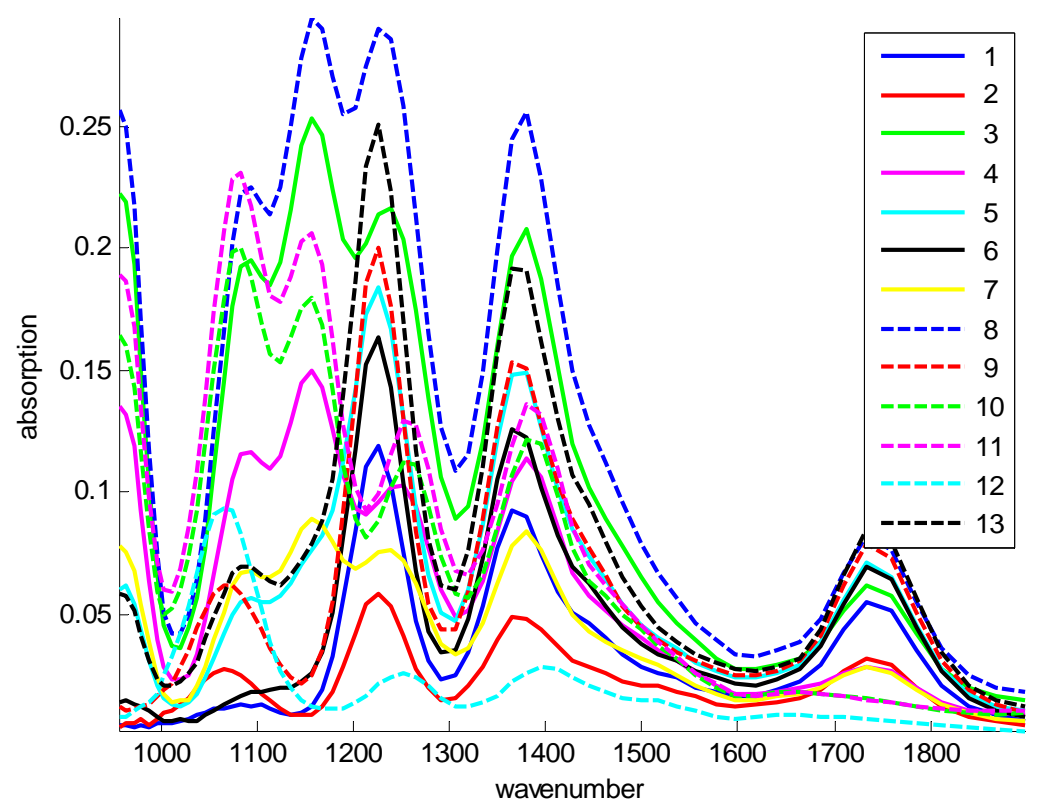

Fig 33. Infrared absorption spectra of thirteen mixtures with dissimilar proportions of acetone, alcohol, witch hazel and water obtained by IRIS.

We can observe that the absorption bands of the different components in the mixture overlap in many instances. Thus, the exact contribution of each component to 
the infrared absorption profile of the mixture cannot be determined simply by visual inspection. Nonetheless, the intensity of the major peaks can serve as evidence for presence and concentration of the different mixture components

\section{V.1.3.1. Calibration results with CLS}

Since the concentration of all interfering spectral species are known, CLS calibration can be performed. As described in section IV.4.1, the mixture composition information (Table 2) is used as the composition matrix $X$, and the recorded mixture spectra is used as our mixture matrix $Y$. We utilize leave-one-out (LOO) cross-validation (See section IV.6.1) to estimate the concentration of acetone for every spectrum in $Y$. The resulting estimate for the concentration of acetone from all mixtures against its true value is illustrated in Fig 34. The calculated MSE error estimate for this model is 0.59 , whereas the correlation coefficient between true and estimated concentrations is 0.9786 . 


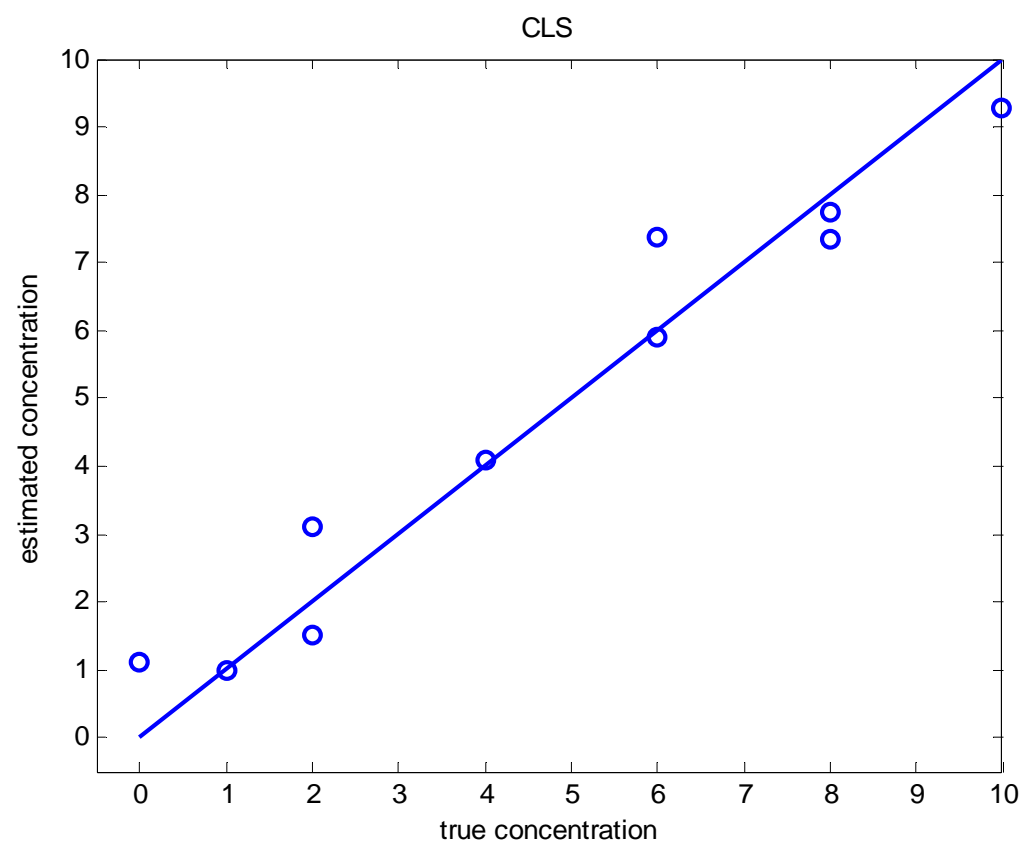

Fig 34. Estimated concentration of acetone from the mixtures described in Table 2 using classical least squares calibration and leave-one-out cross-validation.

\section{V.1.3.2. Calibration results with ILS}

In this experiment, we assume that only the concentration of acetone is known. As described in section IV.4.2, we build the concentration vector for acetone from the composition information in Table 2, and the recorded mixture spectra is used as our mixture matrix $Y$. We utilize leave-one-out (LOO) cross-validation to estimate the concentration of acetone for every spectrum in $Y$. The resulting estimate for the concentration of acetone from all mixtures against their true value is illustrated in Fig 35 . The calculated MSE error estimate for this model is 0.46 , whereas the correlation coefficient between true and estimated concentrations of acetone is 0.9834 . 


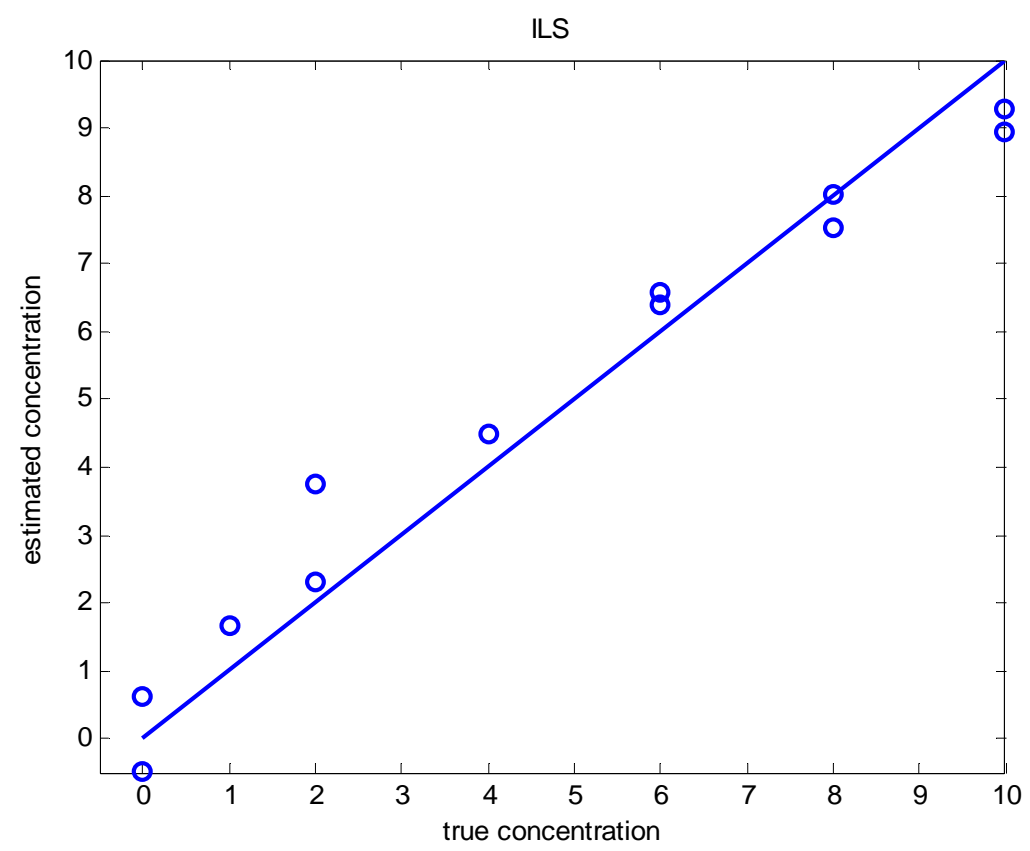

Fig 35. Estimated concentration of acetone from the mixtures described in Table 2 using inverse least squares calibration and leave-one-out cross-validation.

\section{V.1.3.3. Calibration results with PCR}

As before, we build the concentration matrix from the mixture composition information in Table 2, and the mixture matrix $Y$ from the recorded mixture spectra, as described in section IV.4.3. We utilize a two-loop LOO cross-validation process to identify the optimal number of latent vectors for the calibration model. The outer LOO loop evaluates the MSE estimate according to the optimal number of principal components returned by the inner LOO loop. Fig 36 illustrates the average behavior of the MSE estimate as a function of the number of principal components obtained by double loop LOO cross validation; the optimal number of principal components is 4 , which is the same as the number of mixture components. This is attributed to the fact that infrared 
absorption responds linearly and in an equal manner across all wavenumbers to changes in concentration (See section II.4.1.1), thus the total number of degrees of freedom (i.e. sources of variability) equals the number of mixture components, provided that the mixture components have distinct spectra.

In Fig 37, we can observe high similarities between the spectra of the mixture components (See section V.1.2) and the principal component loadings. For instance, the location and shape of the prominent peaks of the first and second principal component loading vector closely resembles the absorption spectrum of IPA and acetone, respectively. The correlation between the principal component scores and the true concentration of acetone is shown in Fig 38; the second principal component is the most correlated with acetone concentration.

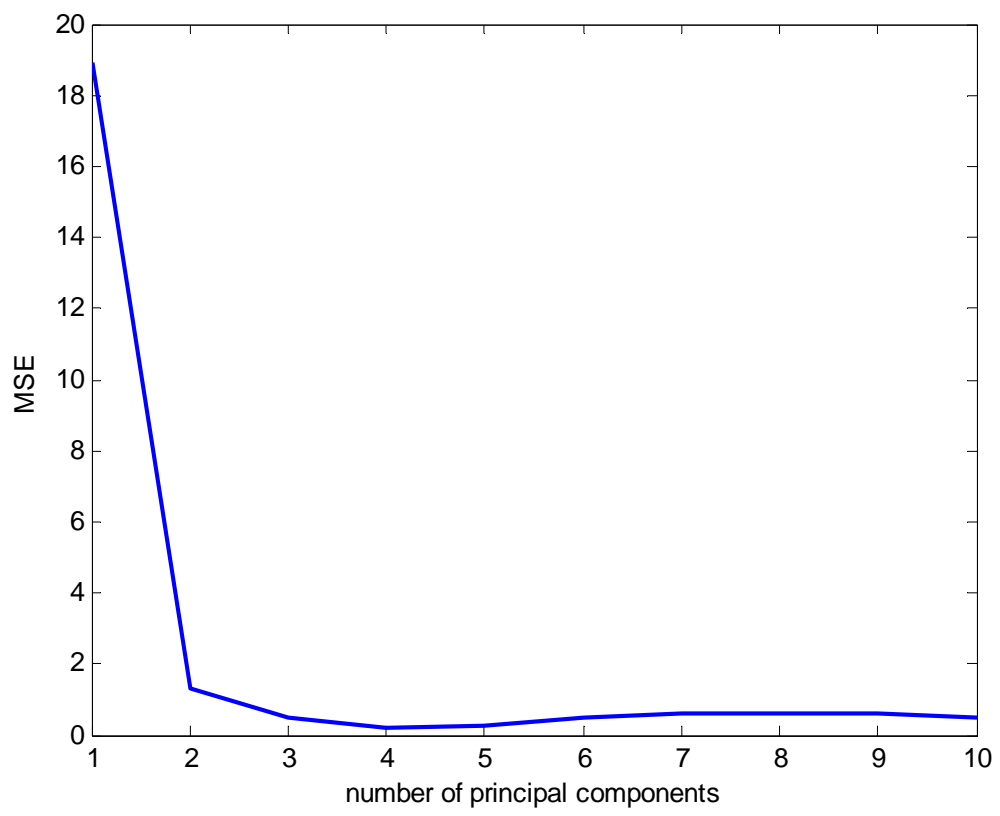

Fig 36. Average behavior of mean square error (MSE) estimate as a function of the number of principal components obtained for PCR via double loop LOO cross validation. 

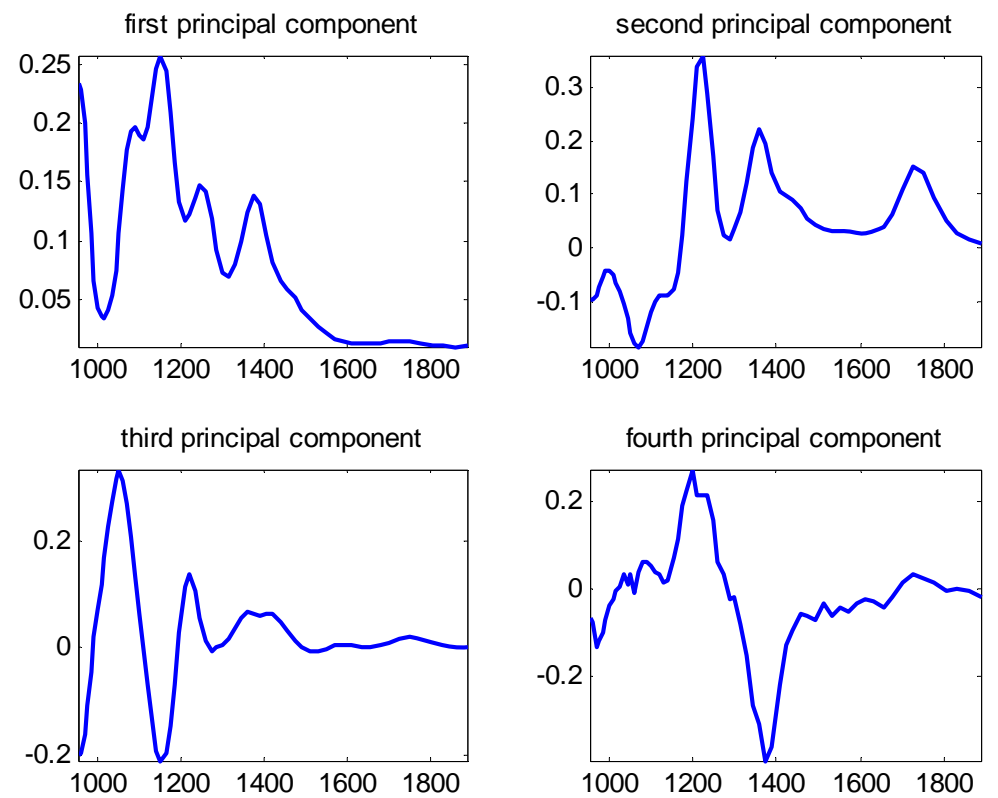

Fig 37. First four principal component loadings.

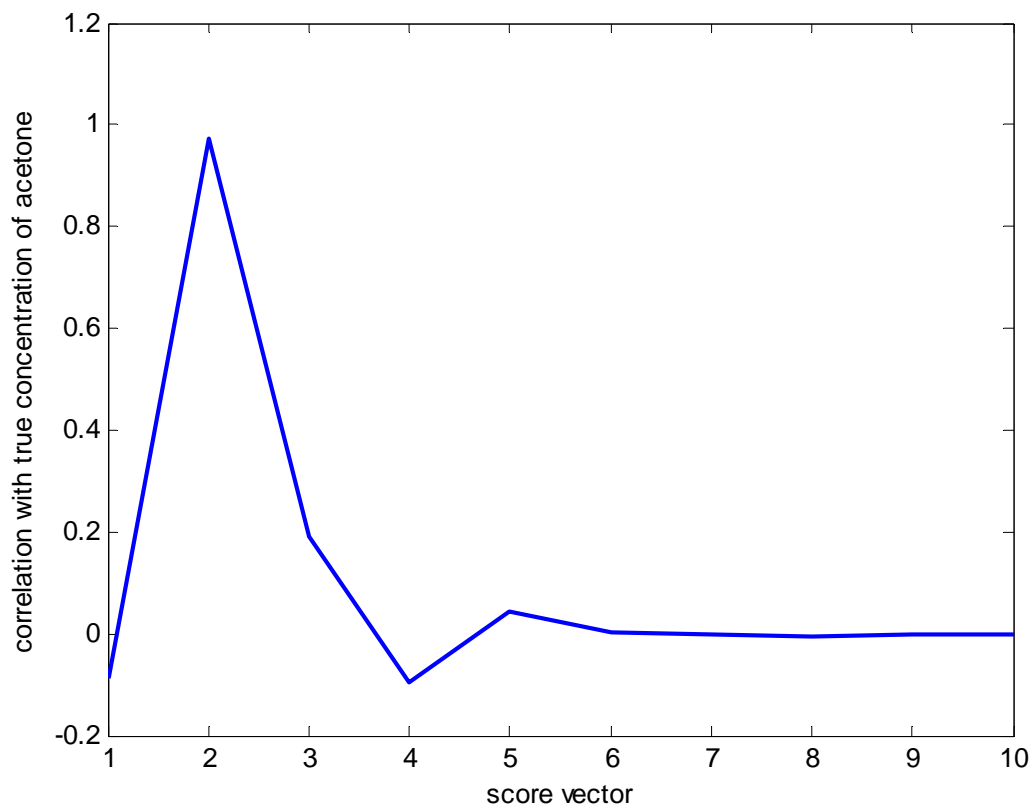

Fig 38. Correlation between principal component score vectors and true concentration of acetone. 
The resulting estimate for concentration of acetone from all mixtures against its true value is illustrated in Fig 39. The calculated MSE error estimate for this model is 0.50 , whereas the correlation coefficient between true and estimated concentrations is 0.9837 .

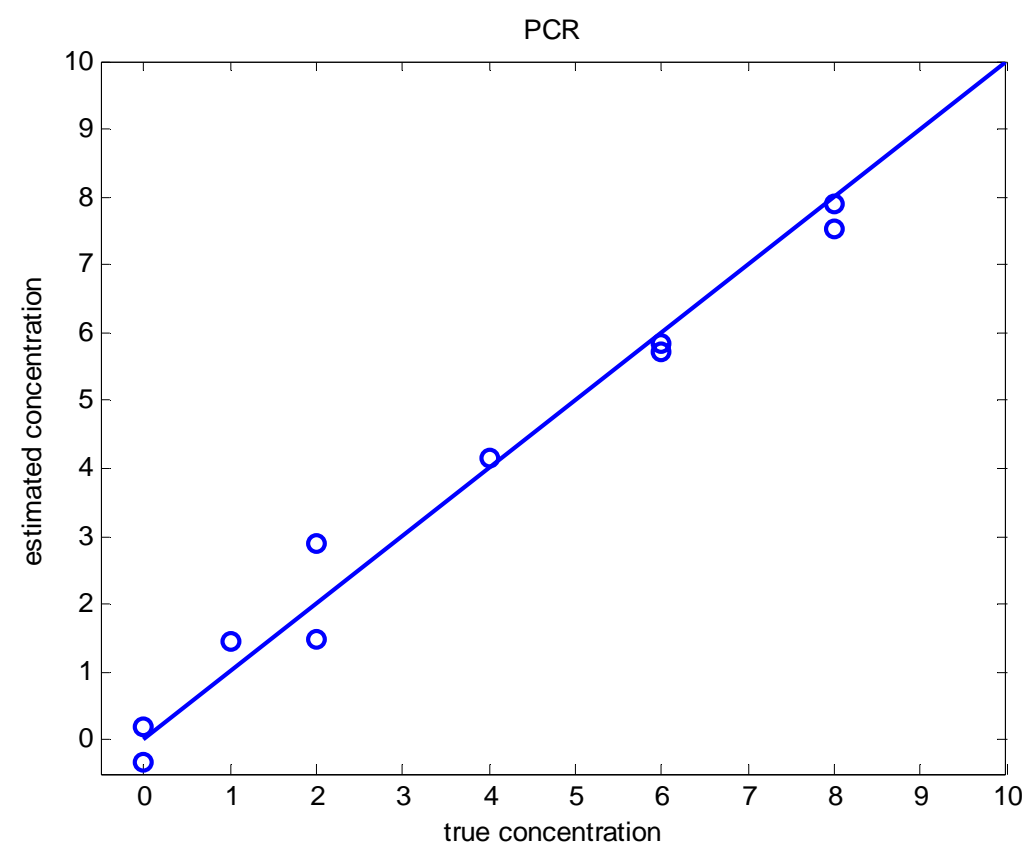

Fig 39. Estimated concentration of acetone from the mixtures described in Table 2 using principal component regression calibration and leave-one-out cross-validation.

\section{V.1.3.4. Calibration results with PLSR}

In this case, we build the concentration matrix from the mixture composition information in Table 2, and the mixture matrix $\mathrm{Y}$ from the recorded mixture spectra, as described in section IV.4.4. We utilize a two-loop LOO cross-validation technique to identify the optimal number of latent vectors for the calibration model. The outer LOO loop 
evaluates the MSE estimate according to the number of latent vectors returned by the inner LOO loop. Fig 40 illustrates the average behavior of the MSE estimate as a function of the number of latent vectors obtained by double loop LOO cross validation; the optimal number of latent vectors is 4 , which is the same as the number of mixture components. This is attributed to the fact that infrared absorption responds linearly and in an equal manner across all wavenumbers to changes in concentration (See section II.4.1.1), thus the total number of degrees of freedom (i.e. sources of variability) equals the number of mixture components, provided that the mixture components have distinct spectra.

In Fig 41, we can observe high similarities between the spectra of the mixture components (See section V.1.2) and the latent vector's loadings. For instance, the location and shape of the prominent peaks in the first latent vectors closely resembles the absorption spectrum of acetone. The correlation between the latent vector scores and the true concentration of acetone is shown in Fig 42. For this model, the first latent vector is the most correlated with acetone concentration. 


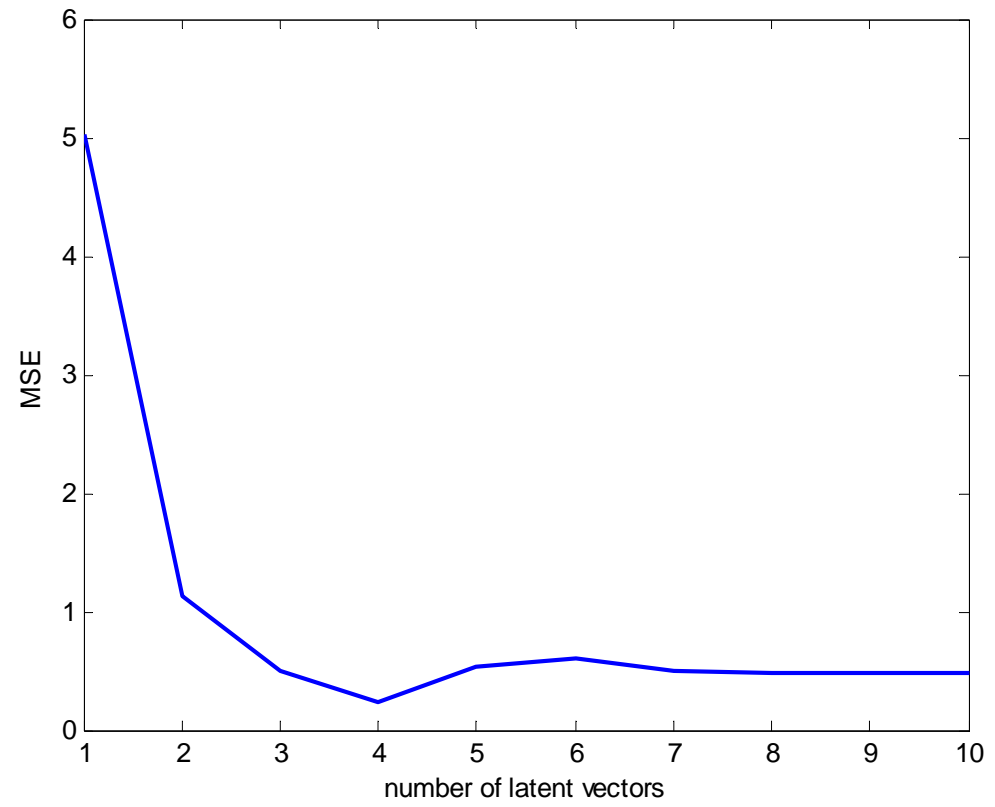

Fig 40. Average behavior of the mean square error (MSE) estimate as a function of the number of latent vectors obtained for PLSR via double loop LOO cross validation.
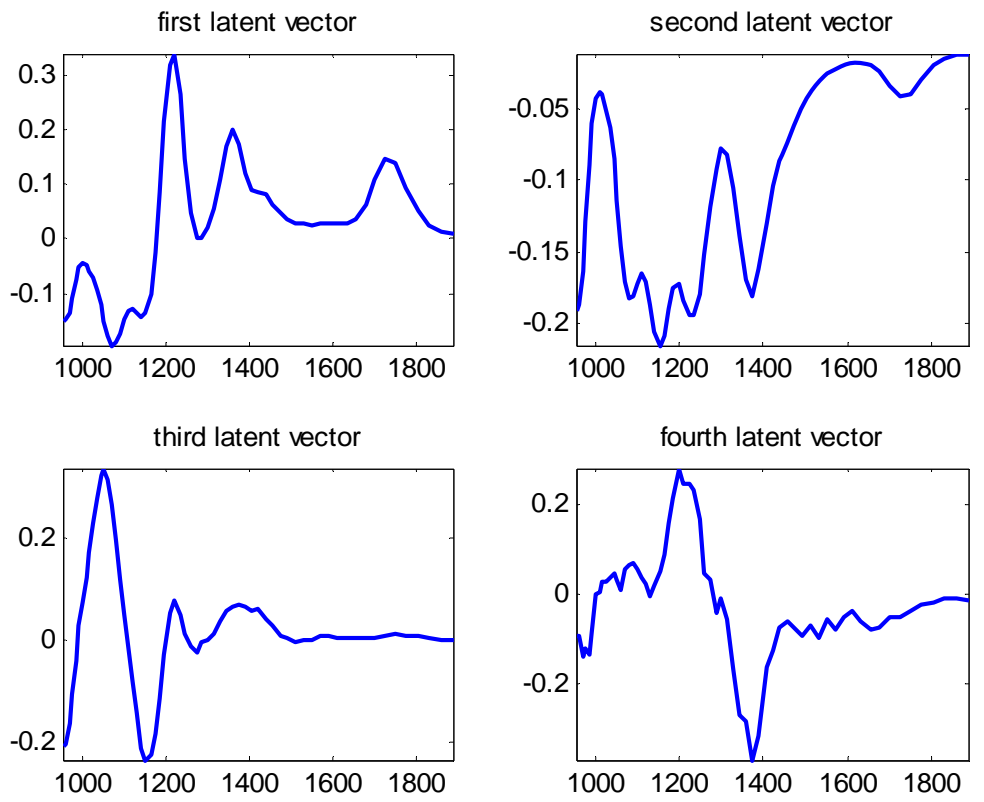

Fig 41. First four latent vectors. 


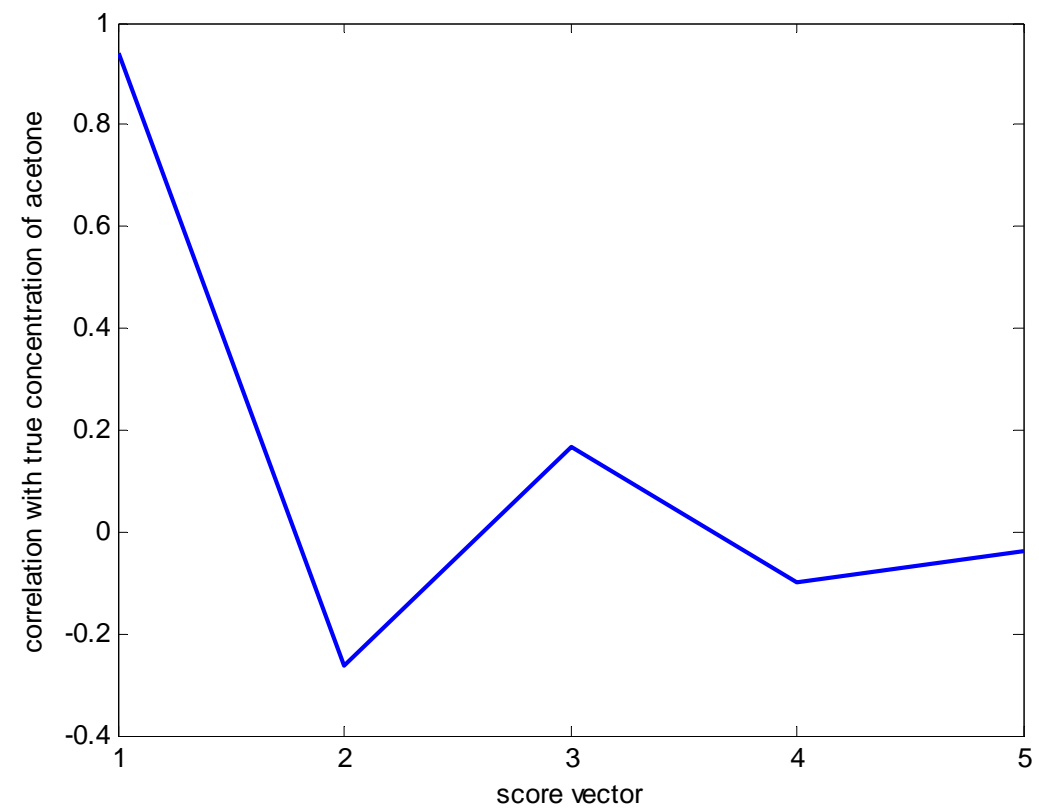

Fig 42. Correlation coefficient between PLSR score vectors and true concentration of acetone.

The resulting estimate for the concentration of acetone from all mixtures against its true value is illustrated in Fig 43. The calculated MSE error estimate for PLSR is 0.47 , whereas the correlation coefficient between true and estimated concentrations for this model is 0.9836 . 


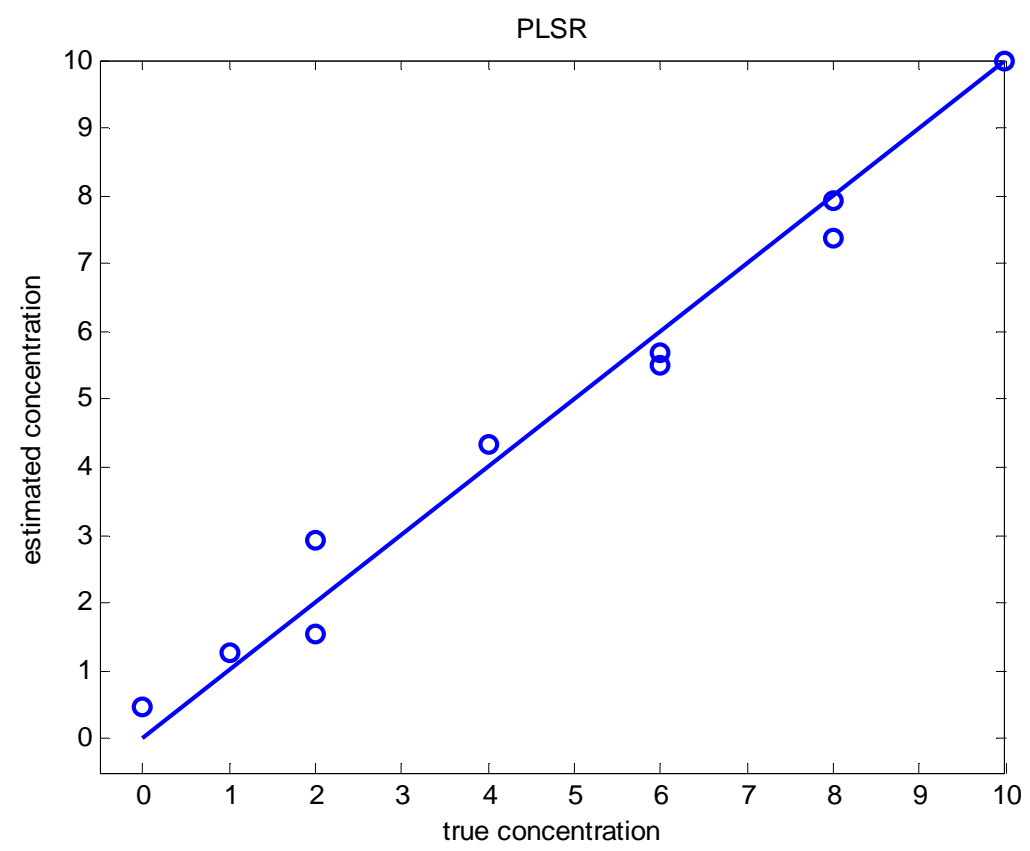

Fig 43. Estimated concentration of acetone from the mixtures described in Table 2 using partial least squares regression calibration and leave-one-out cross-validation.

Using the correlation coefficient between the true and estimated concentrations as a comparison metric, the ILS, PCR and PLSR calibration models demonstrated similar efficiency, outperforming PCR by a small margin.

\section{V.2. Edible oils and fats}

In the second part of this chapter, we evaluate IRIS on two real-world applications of infrared absorption spectroscopy in the edible oils and fats domain: adulteration of oils and trans fatty acid detection. We selected two important applications in this domain because oils and fats have well defined infrared absorption peaks in the LVF region. In this section, we provide a general background on the chemical properties and infrared 
absorption of oils and fats, and defer further the discussion about the two particular applications to sub-sections V.2.2 and V.2.3.

Edible oils and fats are composed primarily of saturated and unsaturated fatty acids. A fatty acid is a carboxylic (i.e. organic) acid with a typically long aliphatic (i.e. open-chain) structure. A fatty acid is said to be saturated if it contains no double bonds between carbon atoms in the aliphatic chain, thus becoming fully saturated with hydrogen atoms. On the contrary, unsaturated fatty acids contain carbon chains with double bonds. If an unsaturated fatty acid contains only one double bond in the aliphatic structure, it is referred to as monounsaturated. Likewise, if it contains more than one double bond between the carbon atoms, it is referred to as polyunsaturated. Unsaturated fatty acids in naturally-occurring edible oils are found in the cis ("on the same side", in Latin) configuration, as illustrated in Fig 44. The presence of double bonds in unsaturated oils makes them more chemically reactive than saturated fats. In fact, the reactivity is proportional to the number of double bonds in the carbon chain. Therefore, unsaturated fatty acids, particularly polyunsaturated, are extremely vulnerable to heat, oxygen and light, which makes them unsuitable for the use in non-perishable food products (Milosevic \& Kocak, 2004).

In order to make oils and fats more resistive to oxidation and rancidity, manufacturers typically employ an industrial process called hydrogenation, which consists of adding hydrogen atoms to unsaturated double bond sites. Specifically, in this process, hydrogen atoms are added to oils at elevated temperatures in the presence of a metal catalyst. The hydrogenation process results in either partially or fully 
hydrogenated fatty acids. Partial and full hydrogenation differ mainly in the number of unsaturated fatty acids in the trans ("on the opposite side", in Latin) configuration left as a byproduct of the hydrogenation process, and in the degree of saturation achieved. The trans fatty acid configuration is also illustrated in Fig 44. Fully hydrogenation yields a higher percentage of saturated fats per volume than partial hydrogenation with almost no trans fatty acids. Furthermore, hydrogenation raises the melting point of oils, and the resulting substance is typically in a semisolid or solid state at room temperature, such as margarine and shortening.

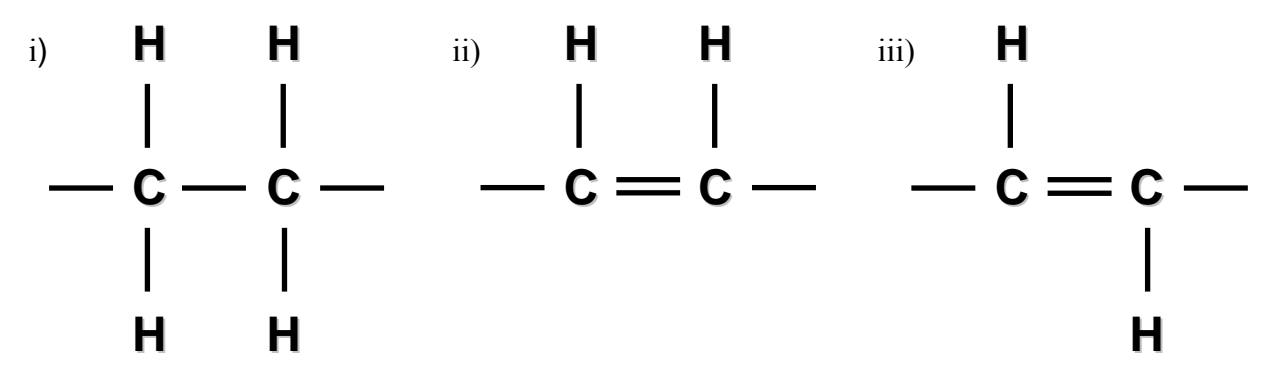

Fig 44. i) Saturated fatty acid. ii) Unsaturated fatty acid in cis configuration. iii) Unsaturated fatty acid in trans configuration.

Most of the commercially-available margarines, spreads and shortenings are manufactured through the partial hydrogenation of soybean oil, and contain a considerable amount of trans fatty acids. The consumption of trans fatty acids increases the risk of coronary heart disease, and has been in the center stage of debate by health activists. We defer further discussions about trans fatty acids, as well as the experimentation with margarine and spreads, to section V.2.3. An overview of the characterization of edible oils by means of mid infrared spectra using IRIS follows. 


\section{V.2.1. The mid infrared spectra of oils and fats}

The mid infrared region contains a wealth of information about the characteristic molecular vibration frequencies of edible oils and fats. Table 3 lists the frequency bands and shoulders of edible oils in the mid infrared region, along with the assigned functional group, mode of vibration and the intensity (Guillen \& Cabo, 1997).

Fig 45 illustrates the infrared absorption spectrum of olive oil in the mid infrared region, obtained by an FTIR instrument, as well as the spectra obtained by IRIS. The numbers atop each major peak indicate the frequency band assignment according to Table 3. Six vibration frequencies seem to strongly contribute to the infrared absorption spectrum obtained by both instruments. Although at smaller resolution, IRIS is able to capture the influence of all the major absorption peaks, and a clear correlation can observed with the high resolution spectra from the FTIR instrument, as indicated by the numbers atop the peaks. 
Table 3 Twenty five important frequency bands and shoulders of edible oils in the mid infrared region, along with the assigned functional group, mode of vibration and the intensity. Adapted from Guillen \& Cabo, 1997.

\begin{tabular}{|c|c|c|c|c|}
\hline No. & Frequency $\left(\mathrm{cm}^{-1}\right)$ & Functional Group & Mode of vibration & Intensity $^{a}$ \\
\hline 1 & $3468(b)$ & $-\mathrm{C}=\mathrm{O}$ (ester) & Overtone & $\mathrm{W}$ \\
\hline 2 & $3025(\mathrm{~s})$ & $=\mathrm{C}-\mathrm{H}\left(\right.$ trans $\left.^{-}\right)$ & Stretching & $\mathrm{vw}$ \\
\hline 3 & 3006 (b) & $=\mathrm{C}-\mathrm{H}($ cis -$)$ & Stretching & $\mathrm{m}$ \\
\hline 4 & $2953(\mathrm{~s})$ & $-\mathrm{C}-\mathrm{H}\left(\mathrm{CH}_{3}\right)$ & Stretching (asym) & $\mathrm{m}$ \\
\hline 5 & 2924 (b) & $-\mathrm{C}-\mathrm{H}\left(\mathrm{CH}_{2}\right)$ & Stretching (asym) & vst \\
\hline 6 & $2853(\mathrm{~s})$ & $-\mathrm{C}-\mathrm{H}\left(\mathrm{CH}_{2}\right)$ & Stretching (sym) & vst \\
\hline 7 & 2730 (b) & $-\mathrm{C}=\mathrm{O}$ (ester) & Fermi resonance & $\mathrm{vw}$ \\
\hline 8 & 2677 (b) & $-\mathrm{C}=\mathrm{O}$ (ester) & Fermi resonance & vw \\
\hline 9 & 1746 (b) & $-\mathrm{C}=\mathrm{O}$ (ester) & Stretching & vst \\
\hline 10 & $1711(\mathrm{~s})$ & $-\mathrm{C}=\mathrm{O}$ (acid) & Stretching & $\mathrm{vw}$ \\
\hline 11 & 1654 (b) & $-\mathrm{C}=\mathrm{C}-(c i s-)$ & Stretching & $\mathrm{vw}$ \\
\hline 12 & 1648 (b) & $-\mathrm{C}=\mathrm{C}-($ cis -$)$ & Stretching & vw \\
\hline 13 & 1465 (b) & $-\mathrm{C}-\mathrm{H}\left(\mathrm{CH}_{2}, \mathrm{CH}_{3}\right)$ & Bending (scissoring) & $\mathrm{m}$ \\
\hline 14 & 1418 (b) & $=\mathrm{C}-\mathrm{H}-($ cis- $)$ & Bending (rocking) & $\mathrm{w}$ \\
\hline 15 & 1400 (b) & & Bending & $\mathrm{w}$ \\
\hline 16 & 1377 (b) & $-\mathrm{C}-\mathrm{H}\left(\mathrm{CH}_{3}\right)$ & Bending (sym) & $\mathrm{m}$ \\
\hline 17 & $1319(\mathrm{~b}, \mathrm{~s})$ & & Bending & $\mathrm{vw}$ \\
\hline 18 & $1238(b)$ & $-\mathrm{C}-\mathrm{O},-\mathrm{CH}_{2}-$ & Stretching, bending & $\mathrm{m}$ \\
\hline 19 & $1163(b)$ & $-\mathrm{C}-\mathrm{O},-\mathrm{CH}_{2}-$ & Stretching, bending & st \\
\hline 20 & $1118(b)$ & $-\mathrm{C}-\mathrm{O}$ & Stretching & $\mathrm{m}$ \\
\hline 21 & 1097 (b) & $-\mathrm{C}-\mathrm{O}$ & Stretching & $\mathrm{m}$ \\
\hline 22 & $1033(\mathrm{~s})$ & $-\mathrm{C}-\mathrm{O}$ & Stretching & $\mathrm{vw}$ \\
\hline 23 & $968(\mathrm{~b})$ & $-\mathrm{HC}=\mathrm{CH}-($ trans -$)$ & Bending out of plane & $\mathrm{w}$ \\
\hline 24 & $914(\mathrm{~b})$ & $-\mathrm{HC}=\mathrm{CH}-\left(\right.$ cis $\left.^{-}\right)$ & Bending out of plane & $\mathrm{vw}$ \\
\hline 25 & $723(\mathrm{~b})$ & $-\left(\mathrm{CH}_{2}\right)_{\mathrm{n}}-,-\mathrm{HC}=\mathrm{CH}-(c i s-)$ & Bending (rocking) & $\mathrm{m}$ \\
\hline
\end{tabular}

${ }^{a} \mathrm{w}$, weak; vw, very weak; m, medium; vst, very strong; st, strong. 

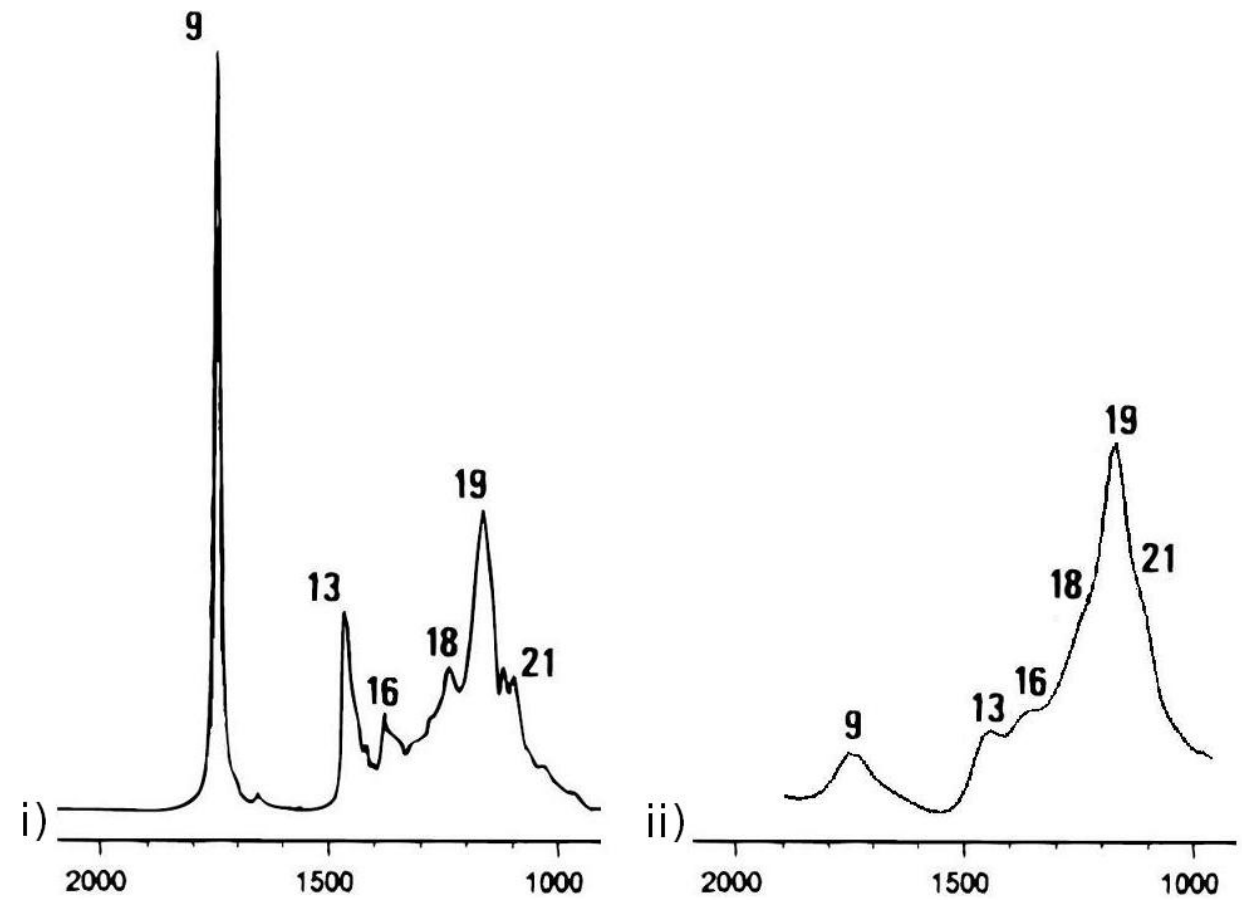

Fig 45. Infrared absorption spectrum of olive oil in the mid infrared region. i) Spectrum obtained by a high resolution FTIR spectroscope (Guillen \& Cabo, 1997). ii) Spectrum obtained by IRIS. The numbers atop each major peak indicates the frequency band assignment in 0 .

\section{V.2.2. Oils}

Five samples of commercially available edible oils were analyzed using IRIS. The objective of this study was to determine if the samples could be discriminated based on their infrared absorption spectra through statistical machine-learning techniques. Among the many applications of oil discrimination using infrared spectroscopy, the determination of authenticity for the prevention of food fraud is an important one. Food fraud consists of the adulteration of food products by substituting something of higher value with something of lower grade, typically for the purpose of economic gain. Food fraud is viewed as a serious issue by the United States Food and Drug Administration 
(FDA), since it can make it difficult for honest companies to compete in the marketplace

and, more importantly, can cause severe health problems for consumers. Because of the superior flavor and potential health benefits of olive oil, it is typically sold at a premium when compared to other vegetable oils. Many approaches for the detection of olive oil adulterations using infrared absorption spectroscopy have been previously reported (Lai et al., 1995; Ulberth \& Buchgraber, 2000). The purpose of this study is to demonstrate IRIS capabilities (and limitations) for the classification of oils.

\section{V.2.2.1. Experimental procedures}

Five samples of commercial products were obtained from local supermarkets. The collection includes all major oils used in the American diet. Table 4 and Table 5 show the composition and class assignment of each sample, respectively.

Table 4 Composition of the samples as provided by the producers.

\begin{tabular}{lrrrrr}
\hline & Canola & Peanut & Corn & Safflower & Olive \\
\hline Saturated Fat (\%) & 7.1 & 17.9 & 14.3 & 7.1 & 14.3 \\
Trans Fat (\%) & 0 & 0 & 0 & 0 & 0.0 \\
Polyunsat Fat (\%) & 28.6 & 35.7 & 57.1 & 71.4 & 10.7 \\
Monosat Fat (\%) & 57.1 & 42.3 & 28.6 & 14.3 & 71.4 \\
\hline
\end{tabular}

Table 5 Samples' producers.

\begin{tabular}{lccccc}
\hline & Canola & Peanut & Corn & Safflower & Olive \\
\hline Producer & Hill Country & LouAna & Hill Country & LouAna & Star \\
\hline
\end{tabular}

IR spectra were recorded from a film of pure oil between two zinc selenide (ZnSe) disks. Specifically, a $0.0125 \mathrm{~mm}$ Teflon spacer was placed between the two ZnSe disks (25 mm diameter), and the gap was filled with a thin (i.e. $6 \mu \mathrm{L})$ oil film. Five 
spectra were collected from each sample. Each spectrum was obtained by placing the liquid cell on the light path, and immediately recording and integrating (i.e. averaging) 250 continuous measurements at a rate of one measurement per second. Because of the relatively low SNR, high degree of perpendicularity of the infrared radiation beams in respect to the infrared windows, and small thickness of the liquid samples, we neglect artifacts that may arise due to scattering of light, and use the Beer-Lambert Law in its original form to calculate absorption.

\section{V.2.2.2. Results and discussions}

Fig 46 illustrates the acquired infrared absorption spectra of all oil samples. It can be observed that, aside from fluctuations due to experimental errors (mainly imprecision in the concentration of the samples), their spectra are nearly identical and practically indistinguishable to the naked eye. 


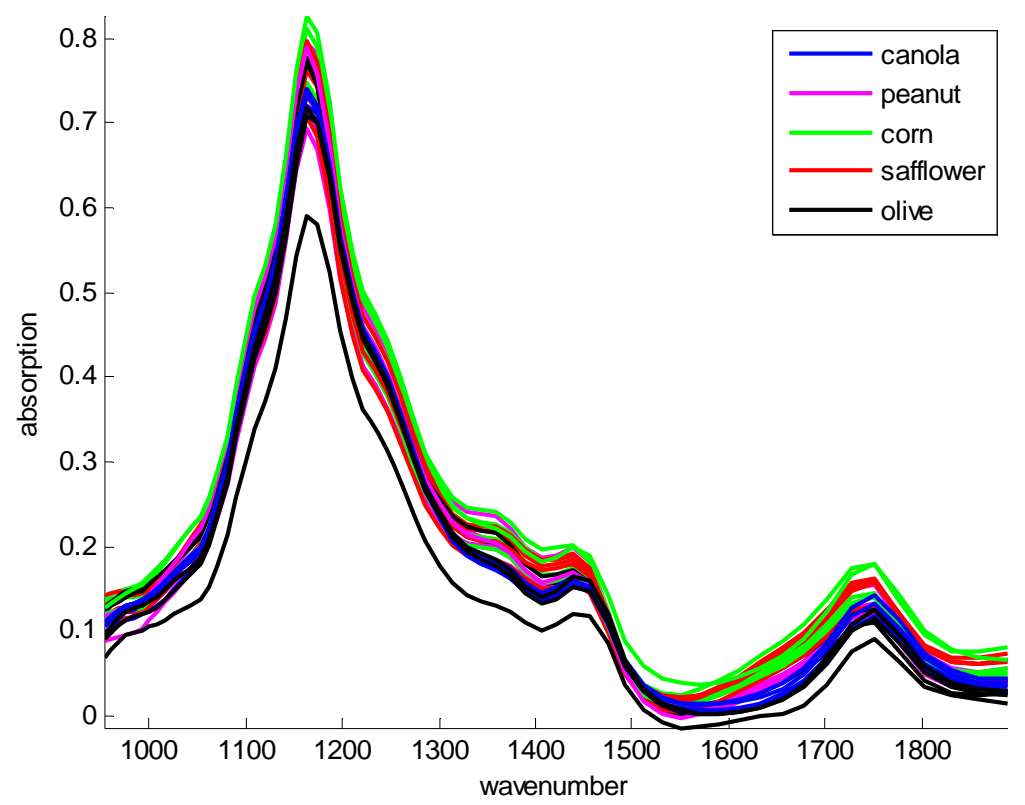

Fig 46. Infrared absorption spectra of five edible oils obtained in IRIS.

However, there are a few subtle differences in these spectra that can be amplified via preprocessing techniques. Since absolute concentration information is irrelevant to the analysis and classification of the samples, we performed vector normalization on each spectrum (See section IV.2.1); thus, all remaining manipulations were performed on normalized spectra. Additionally, in an exploratory manner, we performed differential (See section IV.1.4) and second-derivative spectroscopy. Fig 47 illustrates the result of normalizing the sample spectra to unit vector. Fig 48 shows the spectra after subtracting the canola oil infrared spectrum, and Fig 49 shows the second-derivative of the spectra. 


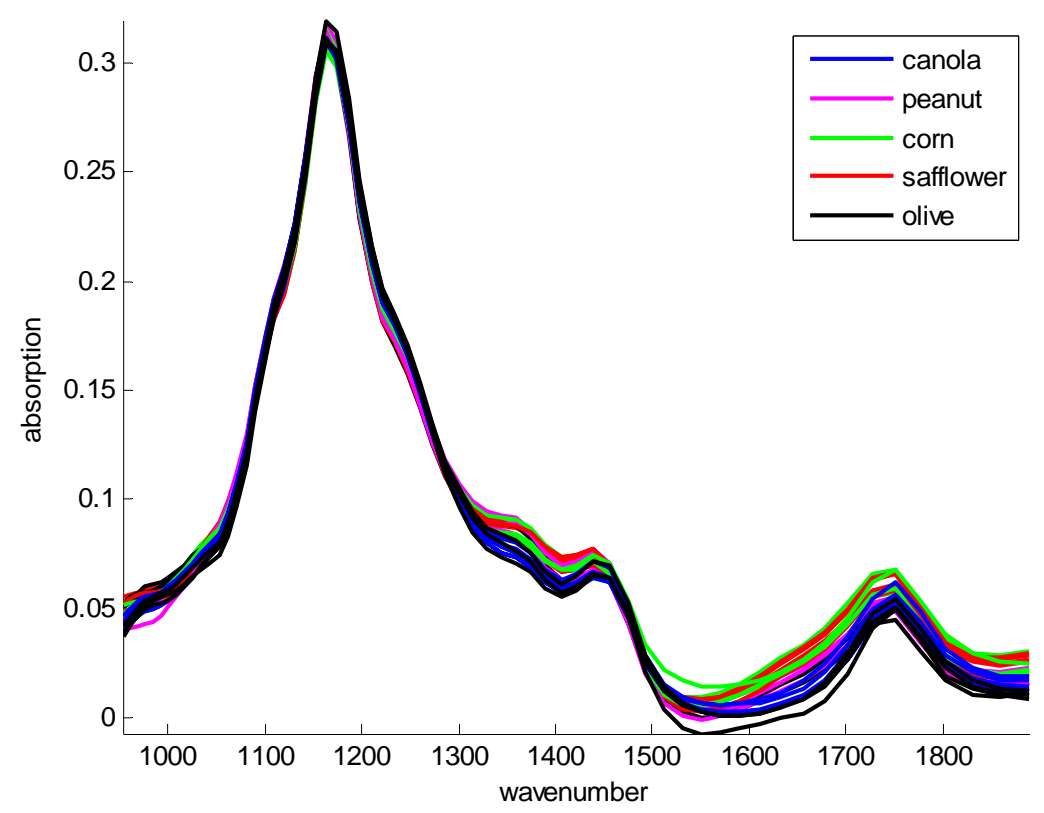

Fig 47. Spectra of edible oils normalized to unit vector.

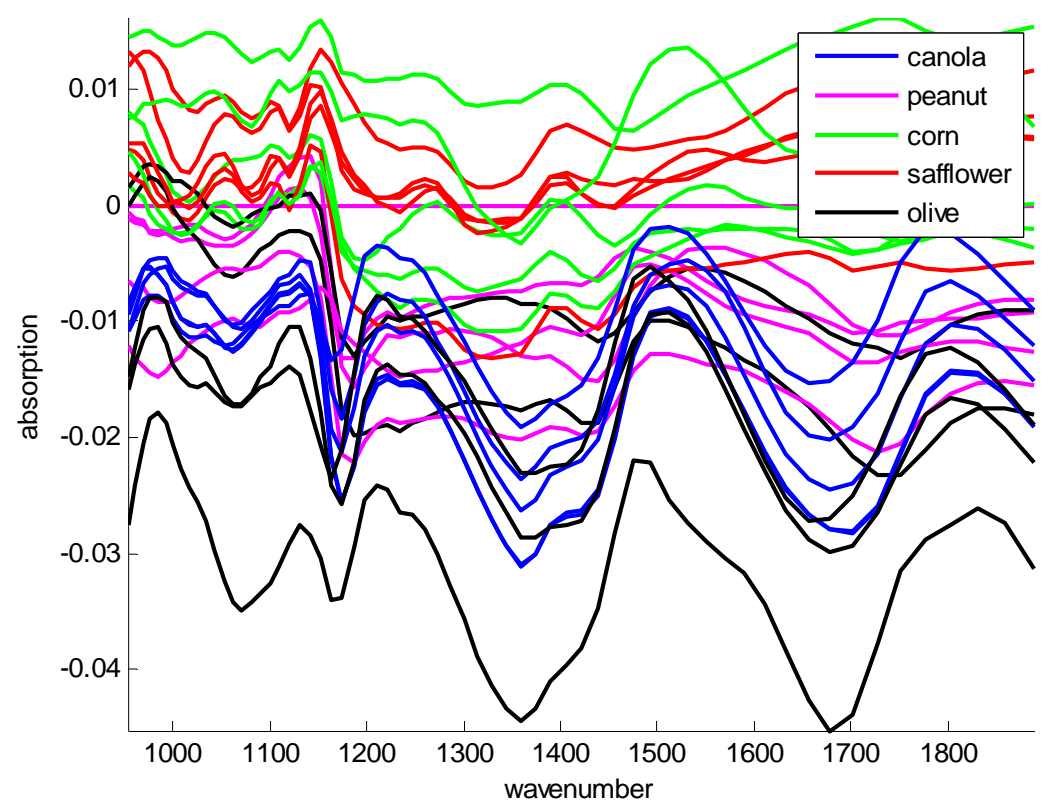

Fig 48. Spectra of edible oils after subtracting the infrared spectrum of peanut oil. 


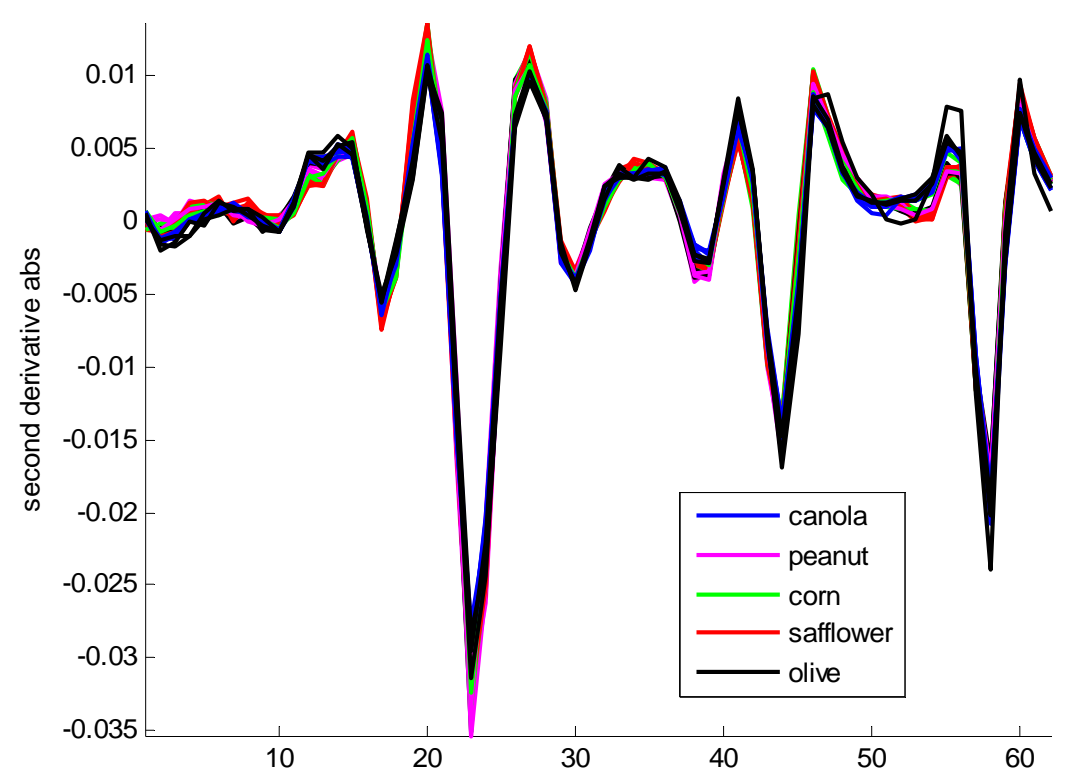

Fig 49. Second derivative spectra of edible oils.

By visual inspection of these three plots, we can observe that the classification problem is not trivial. Without regard for the physicochemical significance, we perform plus-L-minus-R (PLMR) feature selection (See section IV.3.2) to retrieve the best features (i.e. wavenumbers) for classification using proximity (i.e. Euclidean distance) of the spectra as a discriminatory criteria. The PLMR criteria for selecting or removing features are based on the best classification rate obtained via LOO cross-validation after testing every feature available for inclusion and exclusion, respectively. The parameters for the PLMR algorithm were $\mathrm{L}=4$ and $\mathrm{R}=3$, and the algorithm stops when the classification rate decreases or ceases to improve.. The selected features, as well as classification rates with a $1 \mathrm{NN}$ rule are presented in Table 6. 
Table 6 Selected features and their corresponding 1NN/LOO classification rates obtained from PLMR feature selection for the chosen preprocessing techniques. The PLMR parameters are $\mathrm{L}=4$ and $\mathrm{R}=3$.

\begin{tabular}{lcc}
\hline & Selected features & $k N N$ with \\
Vector normalization & $968,1234,1590,1777 \mathrm{~cm}^{-1}$ & LOO classification rate (\%) \\
Difference spectra & $1052,1080,1099,1109 \mathrm{~cm}^{-1}$ & 84 \\
Second derivative spectra & 967,1109 & 100 \\
\hline
\end{tabular}

As shown in Table 6, using two features of the second derivative spectra and 1NN with LOO we are able to obtain complete separability of the samples. It has been reported that the ratio between certain peaks in the mid infrared region contains information about the degree of unsaturation of vegetable oils (Guillen \& Cabo, 1998). Specifically, there is a high correlation (i.e. $>0.99$ ) between the ratio of the bands $A_{20} / A_{21}, A_{6} / A_{14}$ and $A_{6} / A_{21}$, where $A_{x}$ indicates the absorption at the wavenumber given by index $x$ in 0 , and the degree of saturated, monounsaturated and polyunsaturated acyl groups in oils and fats. The absorption bands $A_{20}$ and $A_{21}$ are located in the LVF region, and their peaks correspond to pixels 19 and 20 in the detector array, respectively. By performing feature selection in the second derivative spectra, we find (See Table 6) that the absorption bands around pixels 19, 20 and 21 (i.e. $~ 1109-1130 \mathrm{~cm}^{-1}$ ) contain rich discriminatory information. This can be attributed to differences in the percentage by weight of polyunsaturated acyl groups given by the ratio $A_{20} / A_{21}$, which is highlighted by taking the second derivative of the spectra. Fig 50 shows the second derivative of the spectra at 968 versus that at $1,110 \mathrm{~cm}^{-1}$. From this figure, a clear distinction among classes can be observed. Fig 51 shows the ratio $A_{20} / A_{21}$ on the original spectra. From this figure, clear indication of the degree of correlation between 
the ratio $A_{20} / A_{21}$ with the amount of polyunsaturated acyl groups in the oils can be observed.

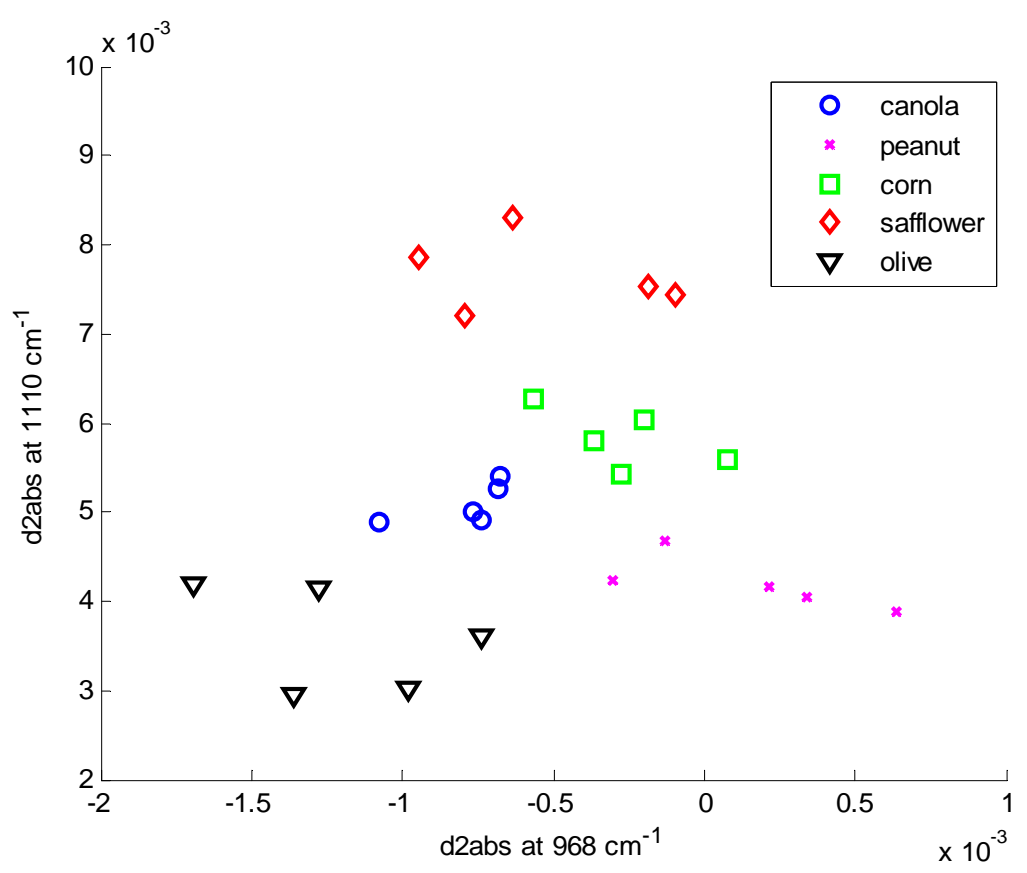

Fig 50. Second derivative at 968 versus $1110 \mathrm{~cm}^{-1}$. 


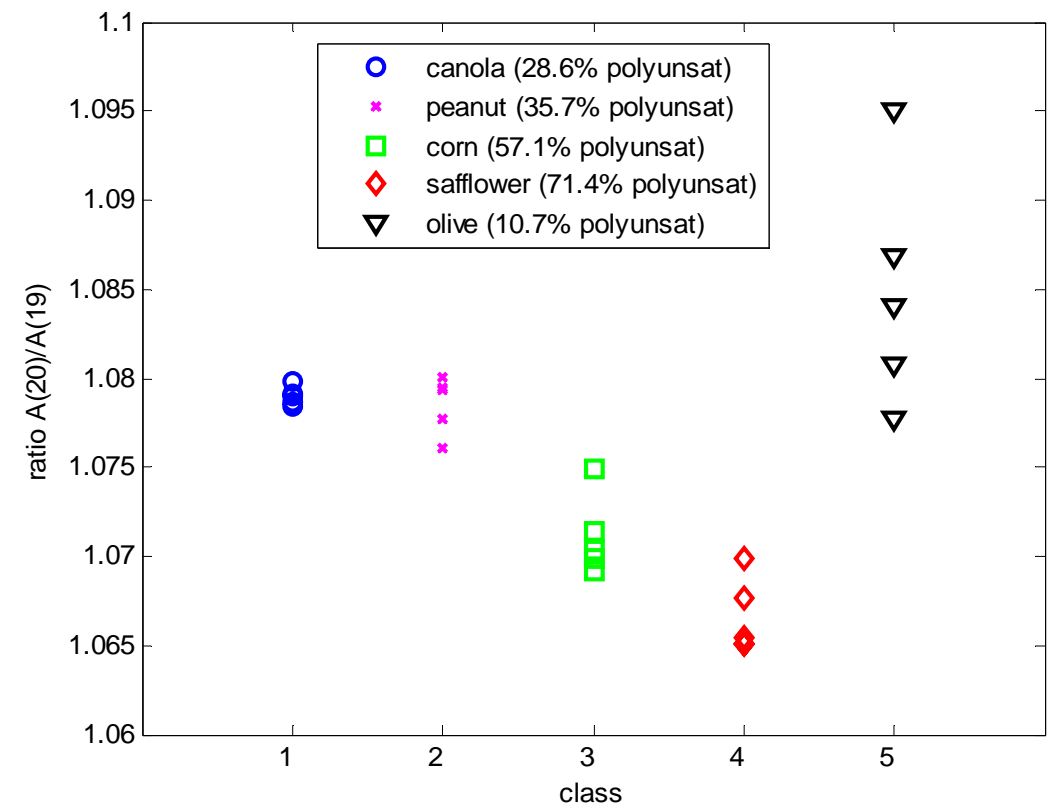

Fig 51. Ratio of absorption $A_{20} / A_{21}$ (See Table 3), which is indicative of the percentage by weight of polyunsaturated acyl groups in edible oils.

Therefore, despite the lower resolution spectra, the discrimination of edible oils is possible with IRIS. Another important application of infrared spectroscopy in the food domain is the detection trans fatty acids (TFA) in manufactured food products. Particularly, in 2006 the FDA has stipulated mandatory labeling of TFA content in food products due to increased concerns of possible health problems associated with the consumption of TFA. In the next section we investigate the potential use of IRIS for the detection of TFA in margarine and spreads.

\section{V.2.3. Trans fatty acids (TFA)}

Recently, there has been a growing concern about the increased risk of coronary heart disease (CHD) due to the consumption of trans fatty acids (TFA). Specifically, TFA has 
been shown to raise low-density lipoprotein cholesterol (LDL-C or "bad" cholesterol), which is a major risk factor of CHD. Based on metabolic studies conducted in 1997 (Ascherio, 1997), it is estimated that approximately 30.000 premature deaths per year in the United States can be attributed to consumption of TFA. TFA health threats have prompted the FDA to require mandatory labeling of TFA content in manufactured food products. The FDA allows manufacturers to choose which methodology to use for obtaining the trans fat content in foods, but indicates that the official methods from the Association of Official Analytical Chemists (AOAC) and the American Oil Chemists Society (AOCS) are considered appropriate and compliant with trans fat nutrition labeling requirements (Milosevic \& Kocak, 2004). The AOAC and AOCS organizations' official methods are based on infrared absorption spectroscopy or gas chromatography. Among the main advantages of infrared absorption spectroscopy over gas chromatography are the reduced sample preparation time and the ability to perform online measurements through the ATR method (See section II.4.2). Online measurements are particularly important for real-time monitoring of heat-induced trans fat formation in food products. However, for offline measurements, transmission methods (See section II.4.1) can also produce reliable results.

In order to comply with FDA labeling requirements, trans fatty acid content below $0.5 \mathrm{~g}$ may be reported as $0 \mathrm{~g}$. Likewise, TFA content must labeled at increments of $0.5 \mathrm{~g}$, and is considered to be misbranded if the nutrient content of the composite samples exceed $120 \%$ of labeling value (Food and Drug Administration, 2003). In this section, we will evaluate the use of IRIS for the detection of TFA content in food products. In 
order to achieve this, we will perform experiments with commercially available margarine and spreads found in two different packages: sticks and tubs. Fig 52 illustrates the two different types of packaging for spread.

i)

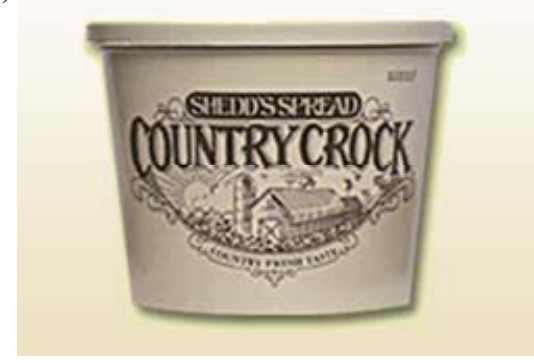

ii)

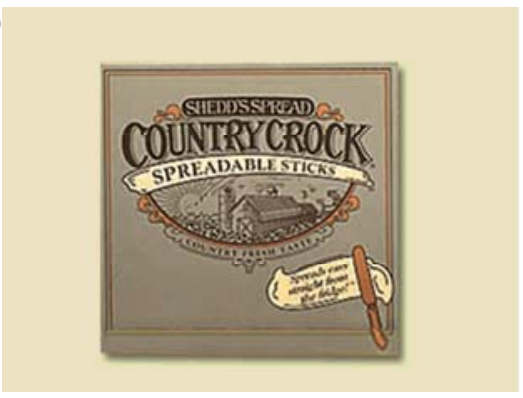

Fig 52. Two types of spread packaging. i) Tub ii) Stick

\section{V.2.3.1. Experimental procedures}

Six samples of commercial products were obtained from local supermarkets. The collection includes three pairs of spread and margarine from three major brands. Table 7 and Table 8 show the class assignment and composition of each sample, respectively. Since margarine and spreads are water-in-oil emulsions, the samples were melted to obtain a samples consisting of only oil.

Table 7 Samples' producers, type and class assignment.

\begin{tabular}{lcccccc}
\hline & HEB & HEB & HEB & HEB & IMP & IMP \\
& $\begin{array}{c}\text { Margarine } \\
\text { in tub }\end{array}$ & $\begin{array}{c}\text { Margarine } \\
\text { in stick }\end{array}$ & $\begin{array}{c}\text { Spread } \\
\text { in tub }\end{array}$ & $\begin{array}{c}\text { Spread } \\
\text { in stick }\end{array}$ & $\begin{array}{c}\text { Spread } \\
\text { in tub }\end{array}$ & $\begin{array}{c}\text { Spread } \\
\text { in stick }\end{array}$ \\
\hline Producer & HEB & HEB & HEB & HEB & Imperial & Imperial \\
Class Assignment & 1 & 2 & 3 & 4 & 5 & 6 \\
\hline
\end{tabular}


Table 8 Composition of the samples as provided by the manufacturers.

\begin{tabular}{|c|c|c|c|c|c|c|}
\hline Class & 1 & 2 & 3 & 4 & 5 & 6 \\
\hline Serving Size $(g)^{a}$ & 14.0 & 14.0 & 14.0 & 14.0 & 14.0 & 14 \\
\hline Total Fat $(g)^{a}$ & 11.0 & 11.0 & 7.0 & 8.0 & 7.0 & 9.0 \\
\hline Saturated Fat $(\%)^{b}$ & 18.2 & 18.2 & 21.4 & 18.7 & 21.4 & 22.2 \\
\hline Trans Fat $(\%)^{b}$ & 9.1 & 27.2 & 7.1 & 25.0 & 7.1 & 27.8 \\
\hline Polyunsat Fat $(\%)^{b}$ & 45.4 & 22.7 & 50.0 & 31.2 & 42.9 & 22.2 \\
\hline Monosat Fat $(\%)^{b}$ & 27.3 & 31.9 & 28.6 & 31.2 & 21.4 & 27.8 \\
\hline
\end{tabular}

IR spectra were recorded from a film of pure oil between two ZnSe disks. Specifically, a $0.0125 \mathrm{~mm}$ Teflon spacer was placed between the two ZnSe disks (25 mm diameter), and the gap filled with a thin (i.e. $6 \mu \mathrm{L})$ film. Three spectra were collected from each sample. Each spectrum was obtained by placing the liquid cell on the light path, and immediately recording and integrating (i.e. averaging) 250 continuous measurements at a rate of one measurement per second. Because of the relatively low SNR, orthogonality of the infrared radiation beams in respect to the infrared windows, and small thickness of the liquid samples, we neglect artifacts that may arise due to scattering of light, and use the Beer-Lambert Law in its original form to calculate absorption.

\section{V.2.3.2. Results and discussions}

Fig 53 shows the infrared absorption spectra of margarine and spread samples. The solid and dashed lines in the figure represent samples in stick form and tub packages, respectively. By carefully examining Fig 53, we can observe a small discrepancy on the fingerprint region between the spectra of samples from the two types of packaging. The distinction is more evident near $968 \mathrm{~cm}^{-1}$, which is precisely where the TFA infrared 
absorption band is centered (See Table 3). The type of packaging used indicates the consistency of the margarine or spread. Tub margarines are softer because they have a higher ratio of liquid oil to hydrogenated fat when compared to stick form. Since the process of partial hydrogenation yields unsaturated fatty acids in the trans configuration (which are in semisolid or solid state at room temperature), typically the more solid the margarine or spread is, the higher the TFA content present.

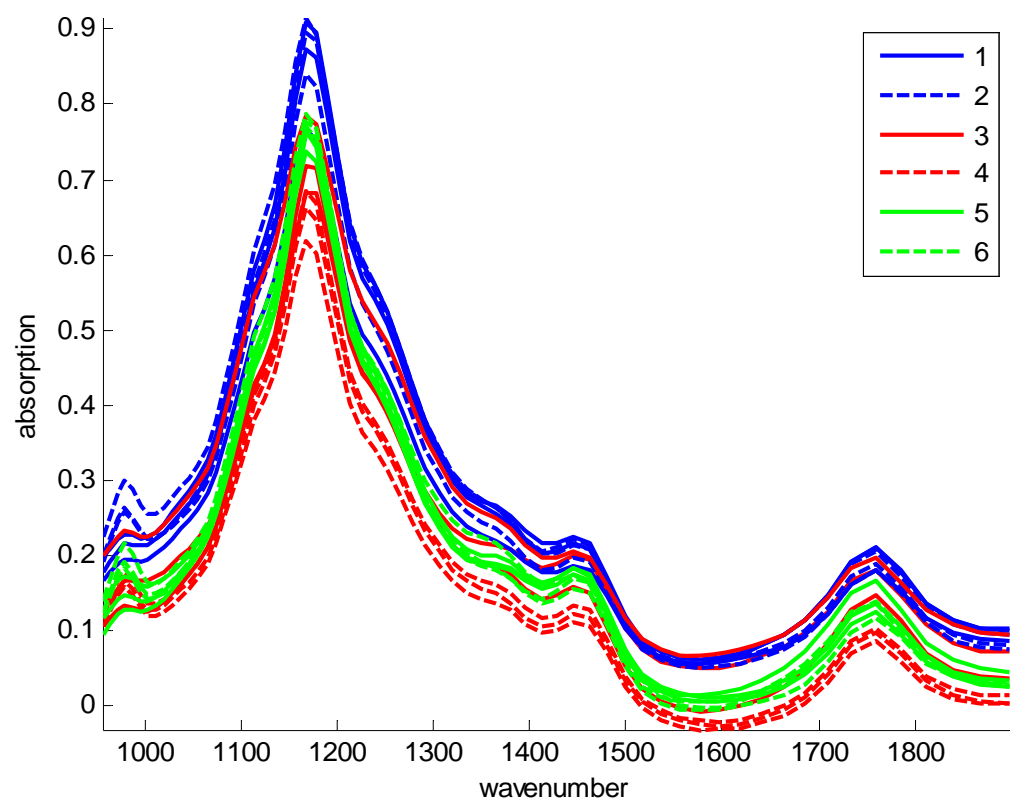

Fig 53. Infrared absorption spectra of margarine and spread samples. Dashed and solid lines represent samples in stick and tub packages, respectively. Three replicates per sample are shown.

We eliminated concentration factors from the sample spectra by performing unit vector normalization. The resulting spectra are illustrated in Fig 54. We can observe that vector normalization improves slightly the visual discernment of samples having different TFA content. 


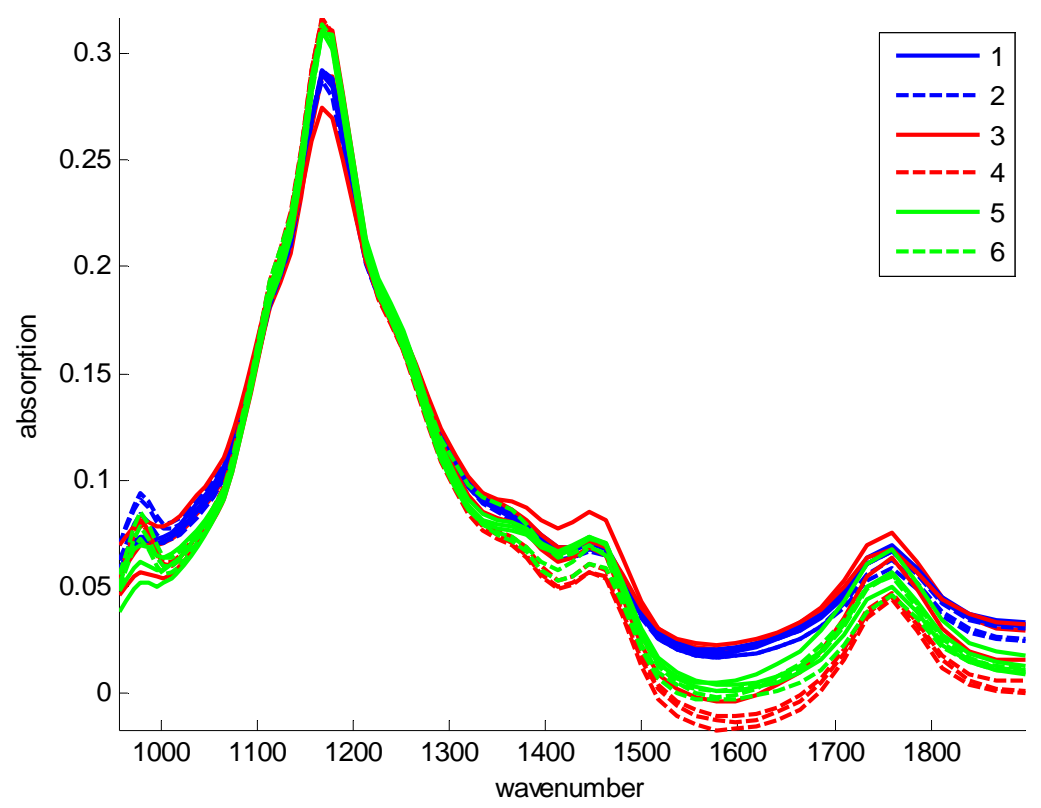

Fig 54. Infrared absorption spectra of margarine and spread samples normalized to a unit vector.

Since the main ingredient of the selected margarines and spreads is soybean oil, we use the difference spectra to remove the contribution of soybean oil from the mixture. The soybean oil spectrum is obtained in the same manner as discussed in section V.2.2.1. However, because of experimental imprecision during the acquisition of the sample spectra, we scale the soybean spectrum to fit each target sample spectrum by means of least squares optimization. The result of this difference spectra method at the TFA absorption band is illustrated in Fig 55. 


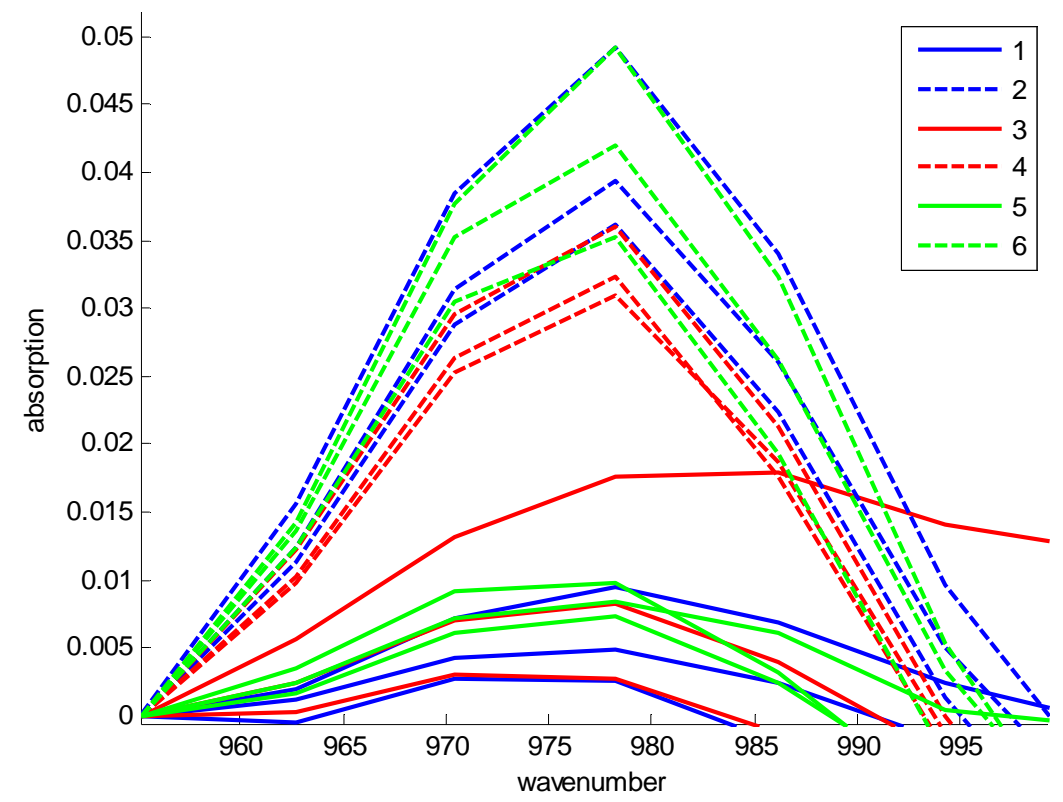

Fig 55. TFA infrared absorption band of margarine and spreads after the subtraction of the infrared spectrum of soybean oil.

The lack of samples with diverse TFA content prevents us from using calibration methods to build and evaluate a good mathematical predictive model for TFA concentration. Assuming that, after performing the difference spectra with respect to soybean oil, the peak at $978 \mathrm{~cm}^{-1}$ shows absorption only due to TFA, we estimate the TFA limit of detection (LOD) in a manner described in section III.3.2:

$$
S=(27.8 \%-7.1 \%) /(0.0421 \mathrm{abs}-0.0085)=616 \% / \mathrm{abs}
$$

where $27.8 \%$ and $7.1 \%$, and are the percentage of TFA content with respect to total fat of classes 5 and 6 . Likewise, 0.0421 and 0.0085 are the average absorptions at $978 \mathrm{~cm}^{-1}$ as illustrated in Fig 55 for classes 5 and 6, respectively.

Using $s_{b i}=0.0053$ (average sample standard deviation of all classes), yields a TFA detection limit as a percentage of total fat of: 


$$
\mathrm{c}_{\mathrm{L}}=3 \times s_{b i} \times S=3 \times 0053 \times 616=9.8 \%
$$

Thus, we estimate LOD for the class with highest fat content per serving (class 1) in terms of weight as follows: $\approx$

$$
\mathrm{LOD}=9.8 \% \times 11 \mathrm{~g} \approx 1 \mathrm{~g}
$$

The relatively high LOD can be attributed to experimental uncertainty, due mostly to imprecise adjustments of the liquid cell, which led to small variations of the path length. The estimated LOD of $1 \mathrm{~g}$ is below (by a factor of 6 ) the necessary LOD for compliance with FDA labeling requirements. However, from Fig 55, it is clear that IRIS can be used for the classification of margarine and spreads based on TFA levels. Based on the available samples, a $1 \mathrm{NN}$ classifier with LOO cross-validation is able to successfully classify all samples into either high-TFA or low-TFA content. These results indicate that the detection of high levels of TFA content in food products is possible using IRIS.

\section{V.3. Conclusion}

We have shown that concentration of acetone in the presence of other absorbing species can be estimated in IRIS using PLSR with an uncertainty of approximately $\pm 0.8 \%$ (derived from MSE), and that oils can be fully separated due to degree of polyunsaturation using pattern classification methods. Furthermore, we have demonstrated that samples of margarine and spreads can be fully classified by IRIS into groups according to their level of trans fatty acid content. 


\section{CHAPTER VI}

\section{CONCLUSIONS AND FUTURE WORK}

In this thesis, we have proposed a low-cost infrared absorption spectroscope based on linear variable filter (LVF) technology for the automated detection of gases and vapors, and the semi-automated detection of liquids. We have characterized the system on a number performance metrics, uncovering its detection limits and resolving power. Furthermore, we have presented calibration methods for building predictive mathematical models for concentration of analytes in a matrix of absorbing species, as well as the associated signal processing techniques for spectral classification.

Despite operating at low-resolution, LVF based spectroscopy is a promising method for the characterization of chemicals. We have demonstrated the use of the instrument for two real-world applications in the edible oils and fats domain: oil adulteration and trans fatty acid detection. We have shown that the spectra of peanut, corn, canola, safflower and olive oil obtained by the instrument can be fully classified via statistical machine learning techniques, and that we can successfully distinguish products (e.g. spreads and margarine) by their levels of trans fatty acid content. Furthermore, when combined with machine learning techniques, the instrument can be used to determine the concentration of an analyte (i.e. acetone) in a matrix of other simple and complex absorbing species (i.e. water, alcohol and witch hazel, in our case) with a high level of precision (i.e. uncertainty of $\pm 0.8 \%$ ). Clearly, this uncertainty estimate depends largely on the characteristic infrared spectra of the samples and matrices, and, therefore, should not be generalized to other calibration problems. 
Nonetheless, these results suggest that LVF based spectroscopy is a viable alternative to traditional high-cost Fourier-Transform Infrared (FTIR) spectroscopy for the quantitative and qualitative analysis of liquids and concentrated gases in carefullyselected niche applications. However, one notable challenge remains: the detection of trace gases.

There are several directions for improvements in the system, the most critical being enhancing the instrument's detection limit for gas measurements. Fortunately, there are several ways in which the detection limit and signal-to-noise ratio (SNR) can be improved:

- By adding a preconcentrator, the concentration of the measurand gas in the gas cell prior to measurement can be increased 10-100 times. Thus, since absorption is linearly dependent on concentration (See section II.4.1.1), detection limit could be improved by a similar factor.

- By replacing the current gas cell by a long-path gas cell, the detection limit of the system could also be improved. This can be attributed to the fact that absorption is linearly dependent on the distance traveled by the infrared radiation (See section II.4.1.1). Furthermore, instrument purging and pressurization with a non-absorbing gas in the LVF region could be useful for avoiding saturation and improving detection for trace gases in the long-path gas cell.

- By employing a pyrolyzer, we can potentially improve sample discrimination. Pyrolyzers provide sample degradation under controlled conditions, with the 
goal of rendering a sample into a more suitable form for a subsequent analytical procedure. For the purpose of trace gas detection, pyrolysis can increase the concentration of the sample's volatile and semi-volatile compounds in the gas cell. Likewise, by fractionated pyrolysis, we could analyze fractions of the sample at different temperature at different times, thus further improving the discrimination of samples.

- By using a circulation pump, we could ensure that the concentration of the measurand gas in the gas approaches the headspace saturation levels.

- By adding a temperature controller at the sample cell, we could improve SNR, particularly for liquid measurements, since infrared spectral response is greatly affected by the temperature of the analyte as well as that of the windows.

A final direction for improvement, though not one we can directly address, is towards the resolving power of the system. Resolution could be enhanced by using a larger array (e.g. 128 or 256) of pyroelectric sensors coupled with higher resolution LVFs (Passerini et al., 2003), with the tradeoff of increased cost. 


\section{REFERENCES}

Abdi, H. (2003). Partial least squares regression (PLS) regression. In: M. Lewis-Beck \& A. Bryman \& T. Futing (eds.), Encyclopedia for research methods for the social sciences (pp. 792-795). Sage, Thousand Oaks, CA.

Adams, M. J. (2004). Chemometrics in analytical spectroscopy. Royal Society of Chemistry, Cambridge, UK.

Andresen, B. F., \& Fulop, G. F. (2005). Infrared technology and applications XXXI, Orlando, FL.

Ascherio, A. (1997). Health effects of trans fatty acids. Am J Clin Nutr 66:1006S-1010S.

Bacon, C. P., Mattley, Y., \& DeFrece, R. (2004). Miniature spectroscopic instrumentation: Applications to biology and chemistry. Review of Scientific Instruments 75(1):1-16.

Badman, E. R., \& Cooks, R. G. (2000). Miniature mass analyzers. J. Mass Spectrom 35:659-671.

Brereton, R. G. (2002). Chemometrics: Data analysis for the laboratory and chemical plant. John Wiley \& Sons Ltd, Chichester, England.

Chou, J. (1999). Hazardous gas monitors: A practical guide to selection, operation and applications. McGraw-Hill Professional, New York, NY.

Couture, M. E. (2001). Challenges in IR optics. Paper presented at the Infrared Technology and Applications XXVII, Orlando, FL.

Duda, R. O., Hart, P. E., \& Stork, D. G. (2001). Pattern classification ( $2^{\text {nd }}$ ed.). John Wiley and Sons, Inc., New York, NY.

EG\&G Princeton Applied Research. (1984). The use of a lock-in amplifier for the detection and measurements of light signals (T-151A-5M/10/84-EG\&G). EG\&G Princeton Applied Research. Wellesley, MA.

Egan, W. J., Morgan, S. L., Bartik, E. G., Merrill, R. A., \& III, H. J. T. (2003). Forensic disctrimination of photocopy and printer toners. II. Discriminant analysis applied to infrared reflection-absorption spectroscopy. Analytical and Bioanalytical Chemistry 376:1279-1285. 
Fisher, R. A. (1936). The use of multiple measurements in taxonomic problems. Annals of Eugenics 7:179-188.

Food and Drug Administration. (2003). Food labeling; Trans fatty acids in nutrition labeling; Consumer research to consider nutrient content and health claims and possible footnote or disclosure statements; Final rule and proposed rule. Federal Register 21 CFR Part 10168 (133):41433-41506.

FT-IR vs. dispersive infrared (2002). TN-00128. Thermo Nicolet Corp. https://www.thermo.com/nicolet.

Fukunaga, K. (1990). Statistical pattern recognition ( $2^{\text {nd }}$ ed.). Academic Press, San Diego, CA.

Geladi, P., \& Kowalski, B. R. (1986). Partial least-squares regression: A tutorial. Anal. Chim. Acta 185:1-17.

Guillen, M. D., \& Cabo, N. (1997). Characterization of edible oils and lard by Fourier transform infrared spectroscopy. Relationships between composition and frequency of concrete bands of the fingerprint region. J am Oil Chem Soc 74:1281-1286.

Guillen, M. D., \& Cabo, N. (1998). Relationships between the composition of edible oils and lard and the ratio of the absorbance of specific bands of their fourier transform infrared spectra. Role of some bands of the fingerprint region. J. Agric. Food Chem 46:1788-1793.

Gutierrez-Osuna, R. (1998). Signal processing and pattern recognition for an electronic nose. North Carolina State University, Raleigh, NC.

Gutierrez-Osuna, R. (2002). Pattern analysis for machine olfaction: A review. IEEE Sensors Journal 2(3):189-202.

Gutierrez-Osuna, R., Nagle, H. T., Kermani, B., \& Schiffman, S. S. (2003). Signal conditioning and preprocessing. In: T. C. Pearce \& S. S. Schiffman \& H. T. Nagle \& J. W. Gardner (eds.), Handbook of machine olfaction. WILEY-VCH Verlag GmbH \& Co., Weinheim, Germany.

Haaland, D. M., \& Thomas, E. V. (1988). Partial least-squares methods for spectral analyses. 1. Relation to other quantitative calibration methods and the extraction of qualitative information. Anal. Chem. 60:1193-1202.

IR Microsystems. (2004). Chopper accessory manual. IR Microsystems. Lausane, Switzerland. 
IR Microsystems. (2006). Detector arrays for grating-based infrared spectrometry solution. IR Microsystems. Lausanne, Switzerland.

JDSU. (2006). Linear variable filters. JDSU. Milpitas, CA.

Lai, Y. W., Kemsley, E. K., \& Wilson, R. H. (1995). Quantitative analysis of potential adulterants of extra virgin olive oil using infrared spectroscopy. Food Chemistry 53:95-98.

Linstrom, P. J., \& Mallard, W. J. (2003). NIST Chemistry WebBook. NIST Standard Reference Database Number 69, National Institute of Standards and Technology (http://webbook.nist.gov).

Loeve, M. (1963). Probability theory ( $3^{\text {rd }}$ ed.). Van Nostrand, New York, NY.

Mark, H., \& Workman Jr., J. (2003). Statistics in spectroscopy ( $2^{\text {nd }}$ ed.). Academic Press, San Diego, CA.

Milosevic, V., \& Kocak, A. (2004). Analyzing trans fats in edible oils and fats using single-reflection ATR-FTIR. American Laboratory(June 2004):30-34.

Nagle, T., Schiffman, S. S., \& Gutierrez-Osuna, R. (1998). The how and why of electronic noses. IEEE Spectrum 35(9):22-34.

Oppenheim, A. V., \& Schafer, R. W. (1996). Discrete-time signal processing (3 ${ }^{\text {rd }}$ ed.). Prentice-Hall, Inc, Englewood Cliffs, N.J.

Passerini, R., Kohli, M., Ryser, P., Seifert, A., Willing, B., et al. (2003). Room temperature spectrometry in the MIR range. Paper presented at the Detectors and Associated Signal Processing, St. Etiene, France.

Persaud, K., \& Dodd, G. (1982). Analysis of discrimination mechanisms in the mammalian olfactory system using a model nose. Nature 299:352-355.

Pinheiro, C., Rodigues, C. M., Schafer, T., \& Crespo, J. G. (2001). Monitoring the aroma production during wine-must fermentation with an electronic nose. Biotechnology and Bioengineering 77(6):632-640.

Pudil, P., Novovicova, J., \& Kittler, J. (1994). Floating search methods in feature selection. Pattern Recognition Letters 15:1119-1125.

Rogalski, A. (2002). Comparison of photon and thermal detector performance. In: M. Henini \& M. Razeghi (eds.), Handbook of infrared detection technologies (pp. 581). Elsevier Advanced Technology, Oxford, UK. 
Rubio, R., Calaza, C., Santander, J., Fonseca, L., Gracia, I., et al. (2004). Optical simulation of MOEMS based tuneable Fabry-Perot interferometer. IEEE Sensors Journal 3:1324-1327.

Rubio, R., Santander, J., Fonollosa, J., Marco, S., Fonseca, L., et al. (2005). Simulation of infrared detector array for gas detection. Paper presented at the ISOEN 2005, Barcelona, Spain.

Schilz, J. (2000). Applications of thermoelectric infrared sensors (thermopiles): Gas detection by infrared absorption. PerkinElmer Optoelectronics $\mathrm{GmbH}$. Wiesbaden, Germany.

Schmidt, W. (2005). Optical spectroscopy in chemistry and life sciences. WILEY-VCM Verlag GmbH \& Co, Weinheim, Germany.

Stuart, B. (2004). Infrared spectroscopy: Fundamentals and applications. John Wiley \& Sons, Ltd, Kent, UK.

Taylor, B. N., \& Kuyatt, C. E. (1994). Guidelines for evaluating and expressing the uncertainty of NIST measurement results. National Institute of Standards and Technology and Technology Note 1297. Gaithersburg, MD.

Taylor, S., Gibson, J. R., \& Srigengan, B. (2003). Miniature mass spectrometry: Implications for monitoring of gas discharges. Sensors review 23(2):150-154.

Thomsen, V., Schatzlein, D., \& Mercuro, D. (2003). Limits of detection in spectroscopy. Spectroscopy 18:112-114.

Tripp, J. S., \& Tcheng, P. (1999). NASA/TP-1999-209545, Uncertainty analysis of instrument calibration and application. Langley Research Center. Hampton, VA.

Ulberth, F., \& Buchgraber, M. (2000). Authenticity of fats and oils. Lipid Sci. Technol 102:687-694.

Vigneau, E., Devaux, M. F., Qannari, E. M., \& Robert, P. (1997). Principal component regression, ridge regression and ridge principal component regression in spectroscopy calibration. Journal of Chemometrics 11:239-249.

Webb, A. R. (2002). Statistical pattern recognition ( $2^{\text {nd }}$ ed.). John Wiley \& Sons, Chichester, England.

Wold, S. (1976). Pattern recognition by means of disjoint principal components models. Pattern Recognition 8:127-139.

Workman Jr., J. (Ed.). (1998). Applied spectroscopy. Academic Press. San Diego,CA. 


\section{APPENDIX A}

\section{PARTS LIST}

Table A.1 Parts list.

\begin{tabular}{|c|c|c|c|c|}
\hline Part & Description & Qty & Price & Vendor \\
\hline $\begin{array}{l}\text { Infrared Emitter (model } \\
\text { IR-12K) }\end{array}$ & $\begin{array}{l}11 \mathrm{~W} \text { thermal emitter with } \\
\text { emissivity of } 0.8 \text {. }\end{array}$ & 1 & $\$ 61$ & $\begin{array}{l}\text { Boston Electronics } \\
91 \text { Boylston St. } \\
\text { Brookline, MA } 02445 \text { USA }\end{array}$ \\
\hline Parabolic Reflector & $\begin{array}{l}\text { Used with the IR-12K thermal } \\
\text { emitter }\end{array}$ & 1 & $\$ 80$ & $\begin{array}{l}\text { Boston Electronics } \\
91 \text { Boylston St. } \\
\text { Brookline, MA } 02445 \text { USA }\end{array}$ \\
\hline Heatsink & Custom built in aluminum & 1 & $\$ 100$ & N/A \\
\hline $\begin{array}{l}\text { Gas Cell (model Beta Gas } \\
\text { Cell) }\end{array}$ & $\begin{array}{l}\text { Cell has a Pyrex body with } \\
\text { two Pyrex filling stems }\end{array}$ & 1 & $\$ 192$ & $\begin{array}{l}\text { Buck Scientific } \\
58 \text { Fort Point Street. } \\
\text { East Norwalk, CT } 06855 \text { USA }\end{array}$ \\
\hline $\begin{array}{l}\text { Liquid Cell (Beta Cell } \\
\text { Kit) }\end{array}$ & $\begin{array}{l}\text { Includes Demountable Cell } \\
\text { Holder, Assorted Teflon } \\
\text { Spacers, Slide Plate Holder }\end{array}$ & 1 & $\$ 50$ & $\begin{array}{l}\text { Buck Scientific } \\
58 \text { Fort Point Street. } \\
\text { East Norwalk, CT } 06855 \text { USA }\end{array}$ \\
\hline $\begin{array}{l}\text { Infrared Transmitting } \\
\text { Window }\end{array}$ & $\begin{array}{l}\text { Zinc selenide window with } \\
\text { anti-reflectance coating }\end{array}$ & 4 & $\begin{array}{l}\$ 106 \\
\text { each }\end{array}$ & $\begin{array}{l}\text { Cradley Crystals } \\
8 \text {, Verhnevolzhskaya nab., } \\
\text { Nizhni Novgorod, 603005, } \\
\text { Russia. }\end{array}$ \\
\hline $\begin{array}{l}\text { Pressure Transducer } \\
\text { (model Honeywell } \\
\text { 24PCCFA6D) }\end{array}$ & $\begin{array}{l}\text { Piezoresistive device with } \\
\text { two-port differential sensing } \\
\text { (0.5-250 psi range) }\end{array}$ & 1 & $\$ 21$ & $\begin{array}{l}\text { Allied Electronics, Inc. } \\
7410 \text { Pebble Drive } \\
\text { Fort Worth, TX } 76118 \text { USA }\end{array}$ \\
\hline Chopper Kit & $\begin{array}{l}\text { Kit contains a mechanical } \\
\text { chopper and driver }\end{array}$ & 1 & $\$ 505$ & $\begin{array}{l}\text { Electro-Optical Products Corp. } \\
\text { 88-65 76th Avenue } \\
\text { Glendale, NY } 11385 \\
\text { U.S.A. }\end{array}$ \\
\hline$\mu$ Array Kit & $\begin{array}{l}\text { Kit contains a 64-pixel } \\
\text { pyroelectric detector coupled } \\
\text { with an LVF }\end{array}$ & 1 & $\$ 4265$ & $\begin{array}{l}\text { IR Microsystems } \\
\text { PSE-C } \\
\text { CH-1015 Lausanne } \\
\text { Switzerland }\end{array}$ \\
\hline $\begin{array}{l}\text { Data Acquisition Card } \\
\text { (DAQ) (model USB- } \\
6009 \text { ) }\end{array}$ & Multifunction I/O & 1 & $\$ 249$ & $\begin{array}{l}\text { National Instruments } \\
11500 \text { N Mopac Expwy } \\
\text { Austin, TX } 78759 \text { USA }\end{array}$ \\
\hline $\begin{array}{l}\text { Oil-less Diaphragm Pump } \\
\text { (model 10D1125-101- } \\
\text { 1052) }\end{array}$ & Pressure/vacuum pump & 1 & $\$ 50$ & $\begin{array}{l}\text { Gast Manufacturing, Inc } \\
\text { P.O. Box } 97 \\
\text { Benton Harbor, MI 49023- } \\
\text { 0097 USA }\end{array}$ \\
\hline $\begin{array}{l}\text { Valves (model EC-2M- } \\
\text { 12) }\end{array}$ & & 9 & $\begin{array}{l}\$ 22 \\
\text { each }\end{array}$ & $\begin{array}{l}\text { Clippard USA } \\
7390 \text { Colerain Ave } \\
\text { Cincinnati OH } 45239 \text { USA }\end{array}$ \\
\hline $\begin{array}{l}\text { Manifold (4-channel, } \\
\text { model 15481-4) }\end{array}$ & $\begin{array}{l}\text { For use with valves (model } \\
\text { EC-2M-12) }\end{array}$ & 3 & $\$ 9$ & $\begin{array}{l}\text { Clippard USA } \\
7390 \text { Colerain Ave } \\
\text { Cincinnati OH } 45239 \text { USA }\end{array}$ \\
\hline $\begin{array}{l}\text { Miscellaneous (gas } \\
\text { circuit) }\end{array}$ & $\begin{array}{l}\text { Includes polyurethane tube, } \\
\text { check valve (model MJCV-1), } \\
\text { air filter }\end{array}$ & 1 & $\$ 20$ & N/A \\
\hline
\end{tabular}


Table A.1 Continued.

\begin{tabular}{llcll}
\hline \multicolumn{1}{c}{ Part } & \multicolumn{1}{c}{ Description } & Qty & Price & \multicolumn{1}{c}{ Vendor } \\
\hline Miscellaneous (electronic & Includes 1 opAmp (model & 1 & $\$ 10$ & Radio Shack \\
parts) & 741) and 6 Regulators (model & & & Culpepper Plaza \\
& ECG261) & & & 1673 Texas Ave. S \\
& & & & College Station, TX 77840
\end{tabular}




\section{VITA}

Felipe Guimarães Nogueira was born in Natal, Brazil. He received a Bachelor of Science in Computer Science from Texas A\&M University in 2002. Upon graduation, he got a position as a Lead Programmer at Resource Studios, Houston, TX where he worked on Computer Aided Facility Management (CAFM) until 2004. He entered the graduate Computer Science program at Texas A\&M University in 2004. In 2005, he joined the Pattern Recognition and Intelligent Sensor Machine's (PRISM) lab in the Computer Science Department at Texas A\&M University to work under Dr. Ricardo GutierrezOsuna in the area of infrared spectroscopy and intelligent sensors. He received a Master of Science degree from Texas A\&M University in 2006. His interests include pattern recognition and intelligent sensors.

Mr. Nogueira may be reached at Schlumberger, 300 Schlumberger Drive, Sugar Land, TX 77478. His email address is fgn6088@cs.tamu.edu. 